Louisiana State University

LSU Digital Commons

1993

\title{
An Algorithm for Supervisory Multivariable Constrained Optimization of Industrial Processes.
}

William E. Daniel Jr

Louisiana State University and Agricultural \& Mechanical College

Follow this and additional works at: https://digitalcommons.Isu.edu/gradschool_disstheses

\section{Recommended Citation}

Daniel, William E. Jr, "An Algorithm for Supervisory Multivariable Constrained Optimization of Industrial Processes." (1993). LSU Historical Dissertations and Theses. 5624.

https://digitalcommons.Isu.edu/gradschool_disstheses/5624

This Dissertation is brought to you for free and open access by the Graduate School at LSU Digital Commons. It has been accepted for inclusion in LSU Historical Dissertations and Theses by an authorized administrator of LSU Digital Commons. For more information, please contact gradetd@lsu.edu. 


\section{INFORMATION TO USERS}

This manuscript has been reproduced from the microfilm master. UMI films the text directly from the original or copy submitted. Thus, some thesis and dissertation copies are in typewriter face, while others may be from any type of computer printer.

The quality of this reproduction is dependent upon the quality of the copy submitted. Broken or indistinct print, colored or poor quality illustrations and photographs, print bleedthrough, substandard margins, and improper alignment can adversely affect reproduction.

In the unlikely event that the author did not send UMI a complete manuscript and there are missing pages, these will be noted. Also, if unauthorized copyright material had to be removed, a note will indicate the deletion.

Oversize materials (e.g., maps, drawings, charts) are reproduced by sectioning the original, beginning at the upper left-hand corner and continuing from left to right in equal sections with small overlaps. Each original is also photographed in one exposure and is included in reduced form at the back of the book.

Photographs included in the original manuscript have been reproduced xerographically in this copy. Higher quality $6 "$ " $\times 9^{\prime \prime}$ black and white photographic prints are available for any photographs or illustrations appearing in this copy for an additional charge. Contact UMI directly to order.

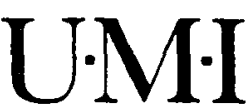

University Microfilms International A Bell \& Howell Informatıon Company 300 North Zeeb Road. Ann Arbor, MI 48106-1346 USA

$313,761-4700 \quad 800,521-0600$ 
Order Number 9419878

An algorithm for supervisory multivariable constrained optimization of industrial processes

\author{
Daniel, William E., Jr., Ph.D.
}

The Louisiana State University and Agricultural and Mechanical Col., 1993 


\footnotetext{
Reproduced with permission of the copyright owner. Further reproduction prohibited without permission.
} 


\title{
AN ALGORITHM FOR SUPERVISORY MULTIVARIABLE CONSTRAINED OPTIMIZATION OF INDUSTRIAL PROCESSES
}

\author{
A Dissertation \\ Submitted to the Graduate Faculty of the \\ Louisiana State University and \\ Agricultural and Mechanical College \\ in partial fulfillment of the \\ requirements for the degree of \\ Doctor of Philosophy \\ in
}

The Department of Chemical Engineering

by

William E. Daniel, Jr.

B.S., Clemson University, 1987

M.S., University of Maryland, 1990

December 1993 


\section{Dedication}

This PhD Dissertation is dedicated to my mother Patricia D. Daniel, my father William E. Daniel, my sister Katherine L. Hanks, my brother Philip K. Daniel, and my grandmother Mrs. Edna H. Johnson, whose support and encouragement have made this achievement possible. 


\section{Acknowledgements}

The author is grateful to Exxon Chemical Company for its financial support and for access to its operational and computer control facilities for testing and data collection, which has been invaluable. The Board of Regents at Louisiana State University is also thanked for its financial support during the course of the research. Dr. Armando B. Corripio is personally acknowledged for his continual encouragement and inspiration throughout the research and writing of the dissertation. 


\section{Table of Contents}

Dedication $\ldots \ldots \ldots \ldots \ldots \ldots \ldots \ldots \ldots \ldots \ldots \ldots$ ii

Acknowledgements $\ldots \ldots \ldots \ldots \ldots \ldots \ldots \ldots \ldots$ ii

List of Tables $\ldots \ldots \ldots \ldots \ldots \ldots \ldots \ldots \ldots \ldots$ vii

List of Illustrations $\ldots \ldots \ldots \ldots \ldots \ldots \ldots \ldots \ldots \ldots \ldots$ viii

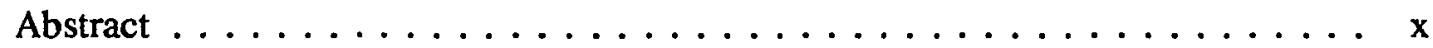

Chapter 1

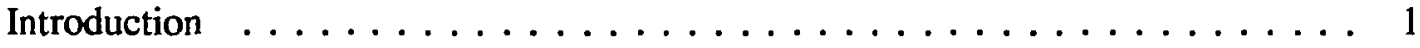

Chapter 2

Literature Review ........................ 4

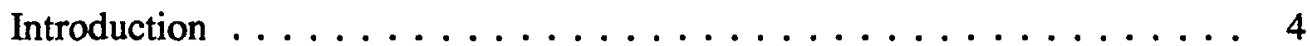

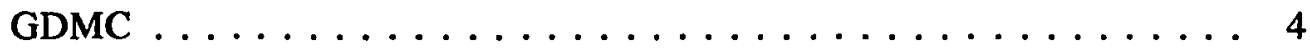

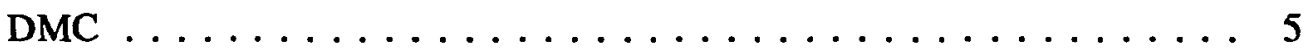

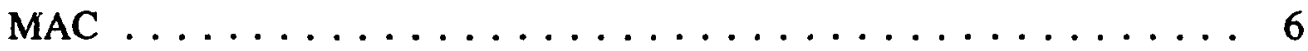

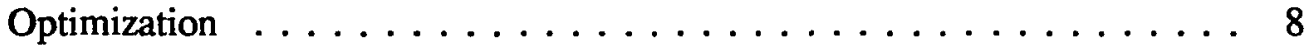

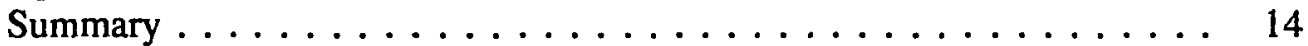

Chapter 3

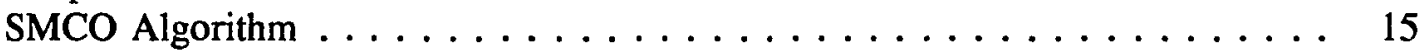

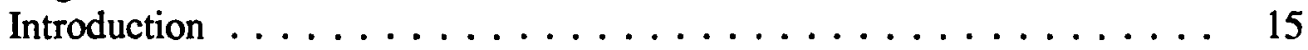

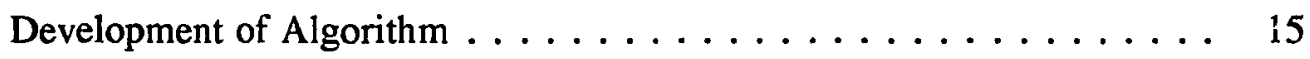

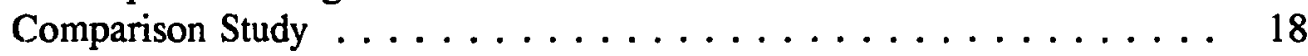

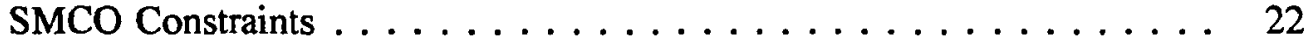

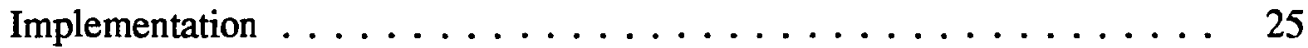

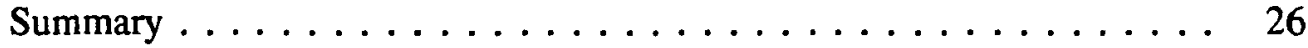

Chapter 4

Distillation Model . . . . . . . . . . . . . . . . . . . 28

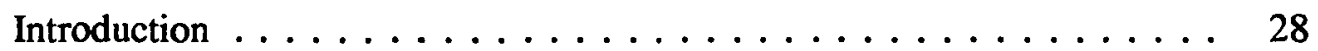

Fenske-Underwood-Gilliland Model ................ 28

Equilibrium/Material Balance Model . . . . . . . . . . . . . . . 30

Summary . . . . . . . . . . . . . . . . . . . . . . 36

iv 


\section{Chapter 5}

Application of the SMCO Algorithm to a Distillation Process . . . . . . . . . 37

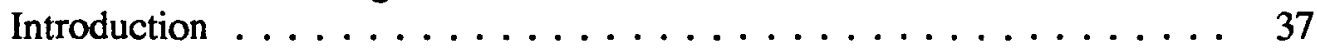

Application of the SMCO Algorithm to a Process . . . . . . . . . . 37

Application to a Demethanizer Column . . . . . . . . . 39

Simulation Results ... . . . . . . . . . . . . . 46

Adaptation of the SMCO Algorithm to a Distillation Train . . . . 49

Simulation Results for Ethylene Purification Train . . . . . . . . 55

Summary . . . . . . . . . . . . . . . . . . . 57

Chapter 6

Additional Examination of SMCO Application to Distillation Process . . . . . 58

Introduction ......................... 58

Independent/Total Mode Difference for Ethylene Purification Train . . 58

Simulation Results with Manipulated Feed to Train . . . . . . . . 60

Columns not Reaching Steady State between Updates . . . . . . . . . 62

Summary . . . . . . . . . . . . . . . . . . . . . 67

Chapter 7

Application of the SMCO Algorithm to Optimize Feed Distribution to Reactors in

Parallel ........................... 70

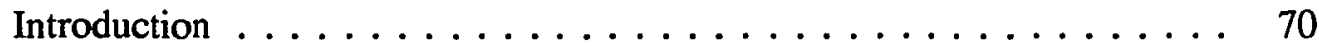

Application of the SMCO Algorithm to Reactor Feed Distribution . . . 72

Reactor Model . . . . . . . . . . . . . . . . . . 72

Simulation Results ................... 78

Summary . . . . . . . . . . . . . . . . . . . 84

Chapter 8

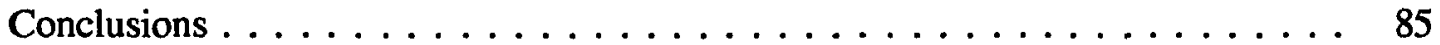

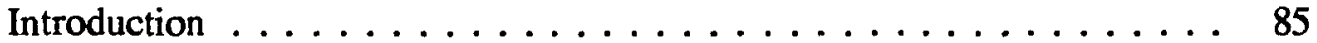

Goals Reached ......................... 85

Contributions ......................... 87

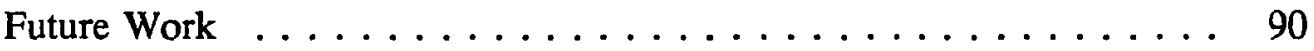

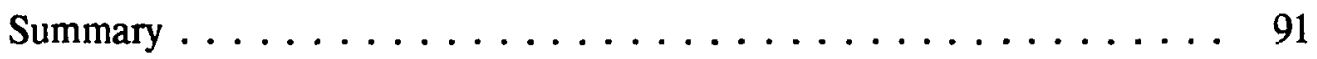

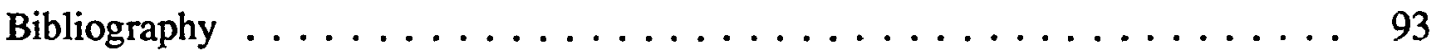

Appendix A

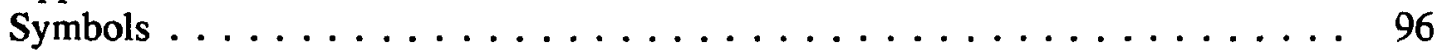

Greek Symbols . . . . . . . . . . . . . . . . . 97 


\section{Appendix B}

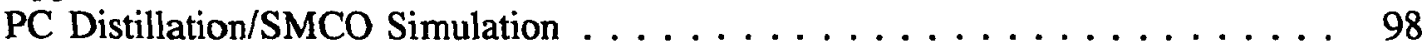

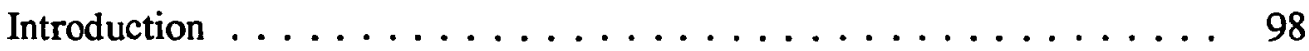

General Information . . . . . . . . . . . . . . . . . . 99

SS Columns Option . . . . . . . . . . . . . . . . . 101

Controllers Options . . . . . . . . . . . . . . . . . . . . 104

Index Definition . . . . . . . . . . . . . . . . . . . . 107

Cost Definition . . . . . . . . . . . . . . . . . . . . . . 109

Run Options . . . . . . . . . . . . . . . . . . . 111

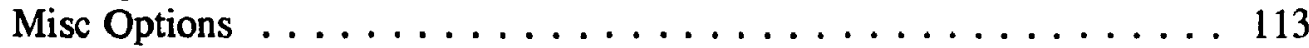

Graphs Options . . . . . . . . . . . . . . . . . . . 113

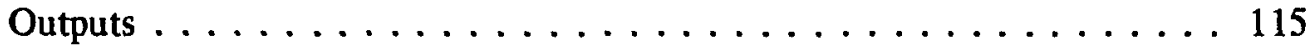

Help Option ............................ 115

Appendix C

PC Reactor/SMCO Simulation . . . . . . . . . . . . . . . . 116

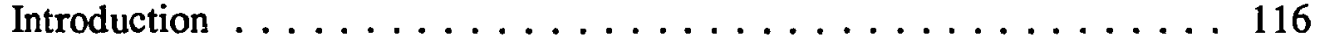

General Information . . . . . . . . . . . . . . . . . . 117

SS Reactor Options . . . . . . . . . . . . . . . . . . . . 118

Controllers Options . . . . . . . . . . . . . . . . . . 119

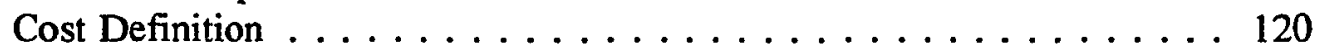

Run Options . . . . . . . . . . . . . . . . . . . . . . 122

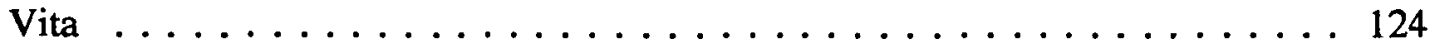

Program Disk 1 of $1 \ldots \ldots \ldots \ldots$. . . . . . . . . . . . . . Packet 


\section{List of Tables}

Table 5-i. Demethanizer Simulation values with feed rate of $10 \mathrm{Klbmole} / \mathrm{hr} \ldots 41$

Table 5-ii. Demethanizer Cost Values . . . . . . . . . . . . . 43

Table 5-iii. Ethylene Train Cost Values . . . . . . . . . . . . . 51

Table 5-iv. Deethanizer and $\mathrm{C}_{2}$ Splitter Values for feed rate of $10 \mathrm{Klbmole} / \mathrm{hr}$. 52

Table 7-i. Feed Breakdown Matrix $\underline{\underline{B}}$ for Reactors . . . . . . . . . . . . . 72

Table 7-ii. Yield Matrix $\underline{\mathrm{g}}$ for Reactor Products $\ldots \ldots \ldots \ldots$

Table 7-iii. Steady State Gain Matrix $\underline{\underline{A}} \ldots \ldots \ldots \ldots \ldots$

Table 7-iv. Reactor Cost Values . . . . . . . . . . . . . . 77 


\section{List of Illustrations}

Figure $3-1$. Penalty Functions for Error . . . . . . . . . . . . . 24

Figure 4-1. Model Distillation Column $\ldots \ldots \ldots \ldots \ldots$. . . . . . . 31

Figure 4-2. Column Trays used in Distillation Model . . . . . . . . . 35

Figure 5-1. Model Distillation Column . . . . . . . . . . . . . . 39

Figure 5-2. Demethanizer Cost as function of $\mathrm{y}_{\mathrm{d} 1}, \mathrm{x}_{\mathrm{b} 2} \ldots \ldots \ldots \ldots$

Figure 5-3. Results of SMCO Operation on Demethanizer: (a) Maximum Vapor Rate and Cost, (b) Compositions, (c) Bottom and Cooling Rates,

(d) Distillate Vapor and Feed Rates ... . . . . . . . . . . . . . 48

Figure $5-4$. Ethylene Purification Train $\ldots \ldots \ldots \ldots \ldots$

Figure 5-5. Train (I)ndependent and (T)otal: (a) Demeth. Max. Vapor \& Costs (b) Demeth. Bottoms \& Dist. Comp. (c) Deeth. Max. Vapor \& Dist. Comp., (d) Deeth. Bottoms \& Cooling, (e) $\mathrm{C}_{2}$ Split. Max. Vapor \& Dist., (f) $\mathrm{C}_{2}$ Split. Comp. . . . . . . . . . . . . . 56

Figure 6-1. (I)ndependent and (T)otal Results with Manip. Feed: (a) Demeth. Maximum Vapor and Costs, (b) Demeth. Overhead Comp. and Bottoms, (c) Deeth. Maximum Vapor and Overhead Comp., (d) Deeth. Bottoms and Cooling Rates $\ldots . \ldots \ldots \ldots \ldots$

Figure 6-2. (I)ndependent and (T)otal Results with three uneven settling times: (a) Demeth. Maximum Vapor and Costs, (b) Demeth. Overhead Comp. and Bottoms, (c) Deeth. Maximum Vapor and Overhead Comp., (d) Deeth. Bottoms and Cooling Rates ... . . . . . . . . . 66

Figure 6-3. (I)ndependent and (T)otal Results for one uneven settling time:

(a) Demeth. Maximum Vapor and Costs, (b) Demeth. Overhead Comp. and Bottoms, (c) Deeth. Maximum Vapor and Overhead Comp., (d) Deeth. Bottoms and Cooling Rates . . . . . . . . . . . 68

Figure 7-1. Distributed Feed Reactor Network showing partial connection of

Figure 7-2. Parallel Reactor Results for Maximizing Profits: (a) Normalized Raw Feeds, (b) Normalized Product Streams, (c) Costs . . . . . . . . .

viii 
Figure 7-3. Parallel Reactor Feed Results for Minimizing Costs: (a) Normalized Raw Feeds, (b) Normalized Product Streams, (c) Costs . .

Figure 7-4. Parallel Reactor Feed Results with extended controller: (a) Normalized Raw Feeds, (b) Normalized Product Streams, (c) Costs . . . 


\begin{abstract}
An algorithm is presented for supervisory optimization of industrial processes that combines the minimization of operating costs with process operating constraints. The supervisory algorithm manipulates the set points of a lower-level control system and the set points are updated at long enough intervals of time so that the process reaches steady state between set point updates. This steady state assumption greatly simplifies the algorithm computations and, more important, significantly reduces the effort required for process identification. This dissertation develops the algorithm and then presents results from its application to a simulated distillation train and reactor feed network. In both applications, the algorithm reduces costs while satisfying changing constraints. In the distillation example, two modes of the supervisory control are identified as "Independent" where the cost is minimized using a dynamic programming approach and "Total" where the whole train is treated as one unit to reduce costs. In some cases where manipulated variables are saturating for a column in the train, the total mode can follow constraint changes whereas the independent mode cannot. For most other test studies, the two modes produce about the same savings and either can be used. Even when steady state is not reached between optimization moves, there is no appreciable difference between the two modes. For the reactor application, the idea of an extended controller is investigated where saturating manipulated variables are removed from the vector set and the optimization algorithm called again. The results show that this extended controller can be economically beneficial and should be used as part of the regular control.
\end{abstract}




\section{Chapter 1}

\section{Introduction}

In these times of tight profit margins and stiff competition, industry must find methods for operating processes at conditions that minimize manufacturing costs and/or maximize production rates. Unlike regulatory control systems, on-line optimization algorithms must take into consideration the complex interactions among several unit operations, and the operating constraints in these processes. On the other hand, the constrained optimization task need only be performed at long enough intervals of time (e.g., hourly or daily) so that it can be assumed the process reaches steady state between the optimization moves. Such an optimization routine also does not have to handle the low level control aspects such as flow control where a PID algorithm works fine. The optimization should act as a supervisory controller which monitors the process and makes adjustments to the low-level controller set points to minimize costs or maximize profits. The ultimate goal of the optimization is to drive the system to a steady state where costs are minimized, but at the same time, operational limits are not violated.

In response to this problem, a supervisory multivariable constrained optimization strategy, SMCO for short, is developed and then applied to various processes to judge its performance. The optimization method should be simple enough to implement on almost any control system yet sophisticated enough to handle complex processes. The strategy should basically allow for real-time cost optimization subject to certain process limits or constraints. The process is assumed 
to reach steady state before the next optimization move is made so simple steady state gain models can be used. These simplified models reduce the task of model identification and make it possible to calculate elements of the gain matrix and cost coefficients on-line from process measurements. The steady-state models also reduce the computations needed in the control algorithm, which leads to a simpler tool for industrial control systems.

The SMCO algorithm should be designed to combine constraint satisfaction with cost reduction and move suppression . However, the constraint violations must take precedence over cost minimization. When there are no constraint violations then SMCO can concentrate on lowering costs. The optimization algorithm should be applicable at different plant levels, such as all the way from a single distillation column to a train of columns. Since SMCO acts in a supervisory role, it can adjust the set points of regulatory controllers which in turn drive the process variables to these set points and keep them there between the updates of the supervisory algorithm.

In the next chapter, the literature is reviewed for similar control and optimization techniques to help classify this new strategy. In Chapter 3 , the algorithm itself is derived and various aspects are explained like how constraints are handled. The research started as an initial proposal to minimize the costs of an ethylene purification train and so a computer simulation was built which incorporated the optimization algorithm. The model used in the simulation is discussed in Chapter 4. Chapter 5 illustrates how the algorithm is set up for a single distillation column 
and then presents simulation results for minimizing costs for a Demethanizer column. The logic is then extended to a train of columns and results are presented for minimizing the costs for an Ethylene Purification train. In Chapter 6, the distillation process is further examined with a different manipulated variable set and with some columns not reaching steady state between the optimization moves. The following Chapter 7 then illustrates how the algorithm can be used to find the optimum feed distribution to a set of parallel reactors to minimize production costs. The concept of an extended controller is introduced and demonstrated using a simulation of parallel reactors. Finally, the conclusions of the research and recommendations for future work are given in Chapter 8. 


\section{Chapter 2}

\section{Literature Review}

Introduction

The Supervisory Multivariable Constrained Optimization (SMCO) algorithm is inspired by a number of control and optimization algorithms that have been reported in the literature. Three of these methods are highlighted here: GDMC or Gain Distribution Multivariable Control (Kennedy, 1975), DMC or Dynamic Matrix Control (Cutler et al, 1979, 1985), and MAC or Model Algorithmic Control (Martin et al, 1984). These control strategies are briefly reviewed and then compared with SMCO. At the end of the chapter, various optimization techniques are discussed in relation to SMCO.

\section{GDMC}

Kennedy originated the idea of a multivariable steady-state control algorithm which minimizes errors of the system (Kennedy, 1975). He called his control algorithm GDMC or Gain Distribution Multivariable Control. The method basically expresses set point deviations as errors using a steady state model of the process. The steady state model consists of a gain matrix which relates the process and manipulated variables, thus the name Gain Distribution control. Constraints are incorporated as one-sided errors through the set point errors, i.e., if a constrained variable is outside its limits a set point error is defined as that limit minus the current 
process value, otherwise the error is given a zero value. GDMC also incorporates an objective function by including the partial of that function with respect to the manipulated variable directly in the steady state error calculation. In one instance, the objective function is a linear cost function. SMCO uses the steady state model approach of GDMC to represent errors and incorporates cost minimization using cost partials with respect to the manipulated variables. The cost may be a linear or nonlinear function of the independent and dependent variables. SMCO also includes move suppression directly in its control equation.

\section{$\underline{\mathrm{DMC}}$}

Dynamic Matrix Control introduced by Charles Cutler and B. L. Ramaker (1979) is a control technique that uses a discrete model of the process to predict the system response and to choose future control moves to eliminate any predicted error. DMC assumes the process can be described by a set of linear difference equations so the process output at any future step can be predicted. The set of equations is usually over-determined which prevents a direct solution, so a least squares criterion is used. Normally the sum of errors squared is minimized. Constraints are incorporated by solving the least squares criterion subject to some process limits (Cutler, Morshedi, and Haydel, 1983).

SMCO is similar to DMC in many respects. Both DMC and SMCO use a least squares approach to solve for the appropriate control moves. Both control techniques incorporate move suppression and constraint handling. Future responses 
can be predicted using past data in both DMC and SMCO. Both methods allow for cost minimization, but the DMC cost optimization process as outlined by Cutler and Hawkins (1988) uses a built-in linear program to find the most economic settings of the manipulated variables to eliminate steady state errors. Both can use off-line identification to get the response coefficient matrix and then use it to calculate the controller gains.

Since SMCO is only concerned with the steady state behavior of the system, its constraint handling could be treated like a one-step prediction and control horizon DMC algorithm where the next step is considered to be at steady state. By considering only one step ahead, the response coefficient matrix of SMCO is much smaller than the standard gain matrix for multi-step DMC. In particular, the SMCO gain matrix only consists of elements, not submatrices, due its steady state approach. For DMC, the gain matrix consists of submatrices where the change in process output has to be considered over some prediction and control horizon. This point is illustrated in Chapter 3 where the SMCO algorithm is developed.

\section{$\underline{\text { MAC }}$}

An European algorithm known as MAC or Model Algorithmic Control is another multivariable predictive control technique (Richalet et al, 1978; Froisy and Richalet, 1986). MAC is also known as IDCOM or IDentification COMmand and Model Predictive Heuristic Control. In MAC, the multivariable process is modelled using impulse-responses. This discrete model is called an internal model. A 
reference trajectory is used to define the closed-loop behavior of the system and drive the system to its set points. The control moves are calculated heuristically, where the system's behavior is predicted and outputs are calculated to match the reference trajectory. Basically, the MAC controller finds the future control moves so the predicted output of the internal model matches as close as possible to the reference trajectory. MAC can handle constraints on the manipulated and controlled variables. The similarities between MAC and DMC are (Martin, 1981):

1) Input/Output model representation of system,

2) Prediction of control variables,

3) Internal model representation of process,

4) Prediction updates with actual measurements,

5) Tuning parameters to dampen control action.

SMCO has many of these same characteristics. Analogies have also been made between DMC, MAC, and dead-beat controllers. For deadbeat control, the desired process response to a specific type of input disturbance is specified. The necessary controller is then found using the closed loop function. It turns out that the deadbeat controller is a special case of MAC and DMC controllers. The deadbeat controller is like the MAC predictive controller when there is no process filter and the output is required to reach the set point in one interval beyond the time delay. A DMC controller is like a deadbeat controller when the control horizon equals the prediction horizon and the weighting of the control moves is small (Martin, 1981). 


\section{Optimization}

Cutler and Perry (1983) sum up real-time optimization in the following steps:

1) Develop process models,

2) Examine current process status,

3) Implement new operating conditions,

4) Check new operating conditions.

They say that on-line optimization is more frugal than off-line because it gives better insight into the actual process dynamics and thus leads to more accurate control. SMCO takes an on-line optimization approach for these reasons.

Garcia and Morari (1981) develop an algorithm for continuous tracking of optimum economic operating conditions using an on-line search technique. The following guidelines are given:

1) Perform on-line experiments to account for unmeasured disturbances and model inaccuracies,

2) Find the optimum faster than the period of disturbances,

3) Account for noisy measurements.

Following these suggestions, an overall economic objective $\mathrm{J}$ is optimized using a linear process model:

$$
\boldsymbol{y}=\underline{\underline{A}} \underline{\underline{u}}
$$

where $y$ represents the process outputs, $\underline{\underline{A}}$ is the steady state gain matrix, and $\underline{\underline{u}}$ represents the optimum control moves. The control moves to minimize $J(y(u), u)$ are obtained using the steepest descent method: 


$$
\left.\underline{u}\right|_{k+1}=\left.\underline{u}\right|_{k}-\left.\mu \nabla_{u} J(y, u)\right|_{k}
$$

where:

$$
\left.\nabla_{u} J(y, u)\right|_{k}=\frac{\left.\partial J(y, u)\right|_{k}}{\partial u}+\frac{\left.\partial J(y, u)\right|_{k}}{\partial y}\left(\frac{d y}{d u}\right)_{k}
$$

The Instrumental Variables (IV) approach is used for parameter estimation since using a least squares criterion can yield biased estimates when noise is correlated to the process (Garcia and Morari, 1981). The IV method uses a matrix of instrumental variables which is not correlated to noise but related to the process outputs and control moves. SMCO borrows the idea of a linear process model but only looks at the steady states of the system and assumes there is no noise correlation. A similar gradient of the economic function is also incorporated into SMCO in finding the optimum.

Moore and Corripio (1991) develop an on-line optimization technique for distillation columns:

1) Adapt distillation model to current conditions,

2) Solve for optimum set points,

3) Send set points to column controllers.

They use a distillation model that relates product recovery to energy consumption through a one-parameter exponential model and material and energy balances on the column. The model parameter is estimated on-line from process measurements. To handle a train of distillation columns, the technique uses a dynamic programming 
approach in which each column in the train is optimized independently, but the cost of each column includes the costs of the columns downstream. An application of SMCO to distillation columns uses the same ideas of expressing the cost in terms of a product recovery term, of using material and energy balances to relate the cost partials to the process variables, and of allowing for a mode to optimize a train of distillation columns using dynamic programming.

Rosendorf (1988) discusses on-line optimization where a set of weight coefficients $w_{1}$ and $w_{2}$ are used to transform a vector problem into a scalar problem. An optimization function is defined which includes energy costs $\left(f_{1}\right)$, production profits $\left(f_{2}\right)$, and functional constraints $\left(g_{i}\left(f_{1}, f_{2}\right)\right)$ with upper bounds $\left(Y_{i}^{\max }\right)$ :

$$
\phi=w_{1} f_{1}+w_{2} f_{2}+\sum_{i=1}^{r} M_{i} E_{i}
$$

The term $E_{i}$ represents one-sided errors where $E_{i}=0$ if the constraint is not violated, i.e., $g_{i}\left(f_{1}, f_{2}\right) \leq Y_{i}^{\max }$, and $E_{i}=Y_{i}^{\max }-g_{i}\left(f_{1}, f_{2}\right)$ if the constraint is violated, i.e., $g_{i}\left(f_{1}, f_{2}\right)>Y_{i}^{\max }$. The $M_{i}$ are positive constants chosen large enough to insure that the inequality constraints are followed. The objective function $\phi$ and its constraints are linear so a linear programming technique known as the simplex method is used to maximize $\phi$. The simplex method starts at a basic feasible solution, one that satisfies all the constraints with nonnegative independent variables, and then steps from one basic feasible solution to the next so that the objective function is always increasing or decreasing (Pike, 1986). The optimization function for SMCO has a non-linear structure and so another technique has to be chosen. 
Koninckx (1988) states that on-line optimization is made up of data reconciliation, model identification, optimization, sensitivity analysis, and parameter estimation. The on-line optimization acts as a supervisory level controller that is situated between the process control loop and the production scheduling. A constrained multivariable search technique known as successive quadratic programming (SQP) is used to find the optimum for a nonlinear boiler network system. The SQP method basically converts a nonlinear problem into an approximate quadratic form with linearized constraints (Pike, 1986). The quadratic approximation is then transformed into an unconstrained linear form using Lagrangian multipliers and their associated properties. A modified Simplex method is then used to solve the set of linear equations. This process is iterated until the optimum is found. SMCO uses a similar supervisory structure but employs a different optimization algorithm. Jang et. al (1987) describe an on-line optimization technique that uses a three tier hierarchy: a production scheduling layer, a optimizing layer, and a regulatory control layer. The scheduling layer sets production rates based on raw materials and product demand. The optimization layer sets the set points of the regulatory level to maximize some objective function. The regulatory level handles the immediate or dynamic control of the process. An objective function is defined which may be any function of the manipulated and process variables. The optimum is found using gradients of the objective function with respect to the manipulated variables and a successive quadratic programming algorithm like the one discussed earlier. The online optimization process can be described as follows: 
1) Take measurements from the plant,

2) Compare actual measurements with model predicted values,

3) If discrepancy is high then execute identification phase,

4) Find optimum settings and transmit back to process,

5) Go back to step 1 .

SMCO uses a optimization technique set up like Jang's but centers on minimizing a cost function which is a nonlinear function of both the dependent and the independent variables. SMCO acts as a supervisor to low level controllers which deal with the immediate control of the process.

McFarlane (1989) uses an optimizing-controller technique where a locally valid steady-state model of the dynamic model is used in combination with sectional linear programming to find the optimum operating conditions. This technique uses a sequence of solutions to local linear problems to solve the original nonlinear problem. Sourander (1984) uses recursive linear programming for a nonlinear olefin-cracking heater problem. The plant is linearized around the current operating point, linear programming is used to find the optimum, and the linearization is repeated as the process moves from one operating point to another. These methods can be categorized as successive linear programming (SLP) methods. Like successive quadratic programming, SLP is a constrained multivariable search technique used for optimizing non-linear processes. The basic approach is to linearize the economic model and the constraints around some starting point then solve the system using the Simplex method (Pike, 1986). The problem is then linearized around the new point 
and the Simplex method is called again. This procedure is repeated until some stopping criterion is reached. The SLP technique could be used for the SMCO algorithm, but a more direct approach is taken as discussed next.

Pike (1986) states there are three components to optimization: a process model, an economic model, and an optimization procedure. Finding the best solution in an optimization is called mathematical programming and is used for steady state systems. The methods for mathematical programming are classified as analytical, geometric programming, linear programming, quadratic programming and convex programming. The two classifications of mathematical programming are direct, where the solution moves from the starting point through improved economic values to the optimum, and indirect, where a set of algebraic equations are solved for the optimum. Analytical and geometric programming are the indirect methods while the rest can be considered direct techniques. The general problem of optimizing a function with ' $M$ ' independent variables subject to ' $N$ ' equality constraints can be solved using the analytical methods of direct substitution, solution by constrained variation, or Lagrangian multipliers if there are more variables than equality constraints $(M>N)$. If the number of unknowns equals the number of equations $(M=N)$ then the independent variables are unique and no optimization is needed. If there are less independent variables than constraint equations $(M<N)$ then the system is over-determined and these techniques alone cannot be used to find the optimum. In direct substitution, the constraint equations are put in terms of the independent variables and then substituted into the optimization function so unconstrained 
optimization techniques can be used. Unconstrained techniques basically consist of differentiating the optimization function with respect to the independent variables, setting these equations to zero, and solving for the optimum point. The technique of Lagrangian multipliers also converts a constrained optimization into an unconstrained one. SMCO uses a direct substitution approach where a process model relating the constraints to the manipulated variables is substituted into the optimization function. A least squares criterion is then used to find the optimum operating point like in DMC.

\section{Summary}

The Supervisory Multivariable Constrained Optimization is an on-line strategy that communicates its moves as set points to lower level regulatory controllers. Constraints are handled through the error term and costs are included through partials with respect to the control moves like in GDMC. The strategy also incorporates move suppression like MAC to prevent large undesirable control changes. The optimization is a combination of minimizing a least squares criterion, as in DMC, and direct substitution of the process model. Linear gain models are used like in DMC but since the process is assumed to reach steady state before the next optimization move, the models are of a much smaller magnitude. This reduction in size helps with the identification phase and with the implementation of the control algorithm. The following chapter derives the SMCO algorithm following these specifications. 


\title{
Chapter 3
}

\section{SMCO Algorithm}

\author{
Introduction
}

This chapter develops the Supervisory Multivariable Constrained Optimization (SMCO) algorithm and then compares it with some other control techniques. The manner in which constraints are handled is discussed next. This section defines the difference between soft and hard constraints. The last part of the chapter discusses how to implement the algorithm both in a real world and simulated application.

\section{Development of Algorithm}

The Supervisory Multivariable Constrained Optimization algorithm minimizes an objective function that combines process constraints, move suppression terms, and the cost function. Such a function with $\mathrm{N}$ constrained variables and $\mathrm{M}$ manipulated variables can be written as:

$$
J=\sum_{i=1}^{N}\left(w_{i} E_{i}\right)^{2}+\sum_{j=1}^{M} \lambda_{j}\left(\Delta U_{j}\right)^{2}+w_{c} C(Y, U)
$$

subject to:

$$
Y_{i}=Y_{i}^{0}+\sum_{j=1}^{M} A_{i j} \Delta U_{j}
$$

where all variables are defined in the symbols section in Appendix A. The first term in the objective function is the weighted sum of the squared errors $\left(E_{i}\right)$, to be 
introduced shortly, which represent the constraints on the dependent variables $\left(Y_{i}\right)$. The second term in the objective function is the weighted sum of the control moves squared. When minimized, this term helps to limit the changes in the control moves $(\underline{\underline{U}})$, that is, incorporates "move suppression" into the scheme. The terms $\lambda_{\mathrm{j}}$ are called "move suppression" parameters and constitute the means by which the algorithm is tuned, just as in MAC and DMC. The last expression in the objective function is the cost function in terms of the process output (dependent) and manipulated (independent) variables. The imposed condition of Equation (3-2) is just a steady-state model where $\mathrm{Y}^{0}$ is the current measurement of the process output and $A_{i j}$ is the appropriate steady state gain between manipulated variable $U_{j}$ and output $Y_{i}$, or $A_{i j}=\partial Y_{i} / \partial U_{j}$ as time goes to infinity. To find the optimum control moves for function $\mathbf{J}$, a technique such as direct substitution, constrained variation, or Lagrangian multipliers could be used as mentioned earlier. However, the problem at hand deals with only inequality constraints and so a least-squares approach is taken as in DMC and MAC. The function $\mathrm{J}$ is treated as the least-squares criterion to be minimized with respect to $\underline{\Delta} \underline{\mathrm{U}}$, resulting in the following set of simultaneous equations:

$$
\frac{\partial J}{\partial \Delta U_{k}}=0, \quad k=1 \text { to } M
$$

The $k^{\text {th }}$ partial can be derived from Equation (3-1) as follows:

$$
\frac{\partial J}{\partial \Delta U_{k}}=2 \sum_{i=1}^{N} w_{i} E_{i} w_{i} \frac{\partial E_{i}}{\partial \Delta U_{k}}+2 \lambda_{k} \Delta U_{k}+w_{c} \frac{D C}{D \Delta U_{k}}
$$


The cost differential in Equation (3-4) can be expressed as:

$$
\frac{D C}{D \Delta U_{k}}=\frac{\partial C}{\partial \Delta U_{k}}+\sum_{i=1}^{N} \frac{\partial C}{\partial Y_{i}} \frac{\partial Y_{i}}{\partial \Delta U_{k}}=\frac{\partial C}{\partial \Delta U_{k}}+\sum_{i=1}^{N} \frac{\partial C}{\partial Y_{i}} A_{i k}
$$

where the steady state relation of Equation (3-2) has been used. The notation $\mathrm{DC} / \mathrm{D} \Delta \mathrm{U}_{\mathrm{k}}$ will be used for compactness in later equations. The steady state model is again used when the error on the constrained variables is re-defined as:

$$
E_{i}=Y_{i}^{\text {set }}-Y_{i}=Y_{i}^{\text {set }}-Y_{i}^{0}-\sum_{j=1}^{M} A_{i j} \Delta U_{j}=E_{i}^{0}-\sum_{j=1}^{M} A_{i j} \Delta U_{j}
$$

where $\mathrm{Y}^{\text {set }}$ is the desired value of the constrained variable $\mathrm{Y}$. Using Equation (3-6) the partial of the error is defined as:

$$
\frac{\partial E_{i}}{\partial \Delta U_{k}}=-A_{i k}
$$

Substituting Equations (3-6) and (3-7) into Equation (3-4), rearranging, and expressing the equation in vector form gives:

$$
\begin{aligned}
& \frac{\partial J}{\partial \Delta \underline{U}}=-2 \underline{\underline{A}}^{T} \underline{\underline{W}}^{T} \underline{\underline{W}} \underline{\underline{E}}^{0}+2 \underline{\underline{A}}^{T} \underline{\underline{W}}^{T} \underline{\underline{W}} \underline{\underline{A}} \Delta \underline{U}+ \\
& 2 \underline{\underline{\Lambda}} \underline{\underline{U}}+w_{c} \frac{D C}{D \Delta \underline{U}}=0
\end{aligned}
$$

Now the control moves are found by solving Equation (3-8) for $\Delta \underline{\mathrm{U}}$ :

$$
\Delta \underline{U}=\left[\underline{\underline{A}}^{T} \underline{\underline{W}}^{T} \underline{\underline{W}} \underline{\underline{A}}+\underline{\underline{\Lambda}}\right]^{-1}\left[\underline{\underline{A}}^{T} \underline{\underline{W}}^{T} \underline{\underline{W}} \underline{E}^{0}-\frac{1}{2} w_{c} \frac{D C}{D \Delta \underline{U}}\right]
$$


The $\Delta \underline{U}$ vector represents the optimum control moves that balance cost minimization with constraint satisfaction using the cost weight factor $w_{i}$. The matrix $\underline{\underline{\Lambda}}$ is a diagonal matrix whose elements are the move suppression parameters $\lambda_{j}$. These elements are to be added to the diagonal elements of $\underline{\underline{A}}^{\top} \underline{\underline{W}}^{\top} \underline{\underline{W A}}$, according to the formula. Since these diagonal elements can have very different magnitudes, it is convenient to scale the move suppression parameters to their corresponding diagonal elements. This is done by multiplying $\left(1+\lambda_{\mathrm{j}}\right)$ times the corresponding diagonal terms of $\underline{\underline{A}}^{T} \underline{\underline{W}}^{\mathrm{T}} \underline{\underline{W A}}$. The scaling operation is represented thus:

$$
\Delta \underline{U}=\left[\underline{\underline{A}}^{T} \underline{\underline{W}}^{T} \underline{\underline{W}} \underline{\underline{A}} \otimes(\underline{\underline{I}}+\underline{\underline{\Lambda}})\right]^{-1}\left[\underline{\underline{A}}^{T} \underline{\underline{W}}^{T} \underline{\underline{W}} \underline{E}-\frac{1}{2} w_{c} \frac{D C}{D \Delta \underline{U}}\right]
$$

The special symbol $\otimes$ means multiply only the diagonal elements of the first matrix times those of the second: $(A \otimes B)_{i j}=A_{i j} B_{i j}$ for $i=j$, and $A_{i j}$ for $i \not j$. The error term $\underline{E}^{0}$ is simply the current error $\underline{E}$ since if no moves are made the system will remain at the same steady state or $\underline{Y}^{0}=\underline{Y}$ when $\Delta \underline{U}=0$.

\section{Comparison Study}

The SMCO optimum control move calculation of Equation (3-9) is similar to those for the multivariable predictive control techniques discussed in Chapter 2. For example, the control equation for Model Algorithmic Control or MAC (Marchetti et al, 1983) is:

$$
\Delta \underline{U}=\left[\underline{\underline{A}}^{T} \underline{\underline{Q}} \underline{\underline{A}}+\underline{\underline{R}}\right]^{-1} \underline{\underline{A}}^{T} \underline{\underline{Q}} \underline{\underline{E}}^{0}
$$


where $Q$ and $R$ are positive-definite weighting matrices for the error terms and the control moves, respectively. DMC's control move calculation to minimize the square of the deviation between predicted output and set point trajectory (Georgiou et al, 1988) can be written as:

$$
\Delta \underline{U}=\left[\underline{\underline{A}}^{T} \underline{Q}^{T} \underline{\underline{Q}} \underline{\underline{A}}+k^{2} \underline{\underline{I}}\right]^{-1} \underline{\underline{A}}^{T} \underline{\underline{Q}}^{T} \underline{\underline{Q}} \underline{\underline{E}}^{0}
$$

where $\mathrm{Q}$ is a diagonal weighting matrix for the error terms, $\mathrm{k}$ is a scalar move suppression factor, and I is the identity matrix. The SMCO algorithm, however, normalizes its variables relative to their corresponding ranges, $\left(Y^{\max }-Y^{\min }\right)$ for the dependent variables and $\left(U^{\max }-U^{\min }\right)$ for the manipulated variables, to maintain the terms in the objective function to approximately the same magnitudes. In terms of minimizing set point violations, SMCO's formulation is basically the same as that of DMC and MAC.

A major difference between SMCO and DMC or MAC is the direct incorporation of the cost function into the control move calculation. MAC does not address the economic side of optimization. However, DMC incorporates economics through a linear program or model of the process (Tran et al, 1989; Tomlins and Thieme, 1989). To maintain control and minimize cost in DMC applications, the following procedure is followed (Tran et al, 1989):

1) Predict future responses of the dependent variable based on past independent variables. 
2) Use a linear program and predicted steady state errors of step 1 to calculate the most economic steady state in terms of the controller's manipulated variables.

3) Use DMC controller to minimize the error over the control horizon in getting to the economic steady state of step 2 .

SMCO incorporates the cost's effect by including the change in cost per change in manipulated variable or the DC/DU term. SMCO allows any formulation of the cost function as long as the $\mathrm{DC} / \mathrm{D} \underline{\mathrm{U}}$ terms are defined. This set-up provides great flexibility yet easy computation.

Another difference between SMCO and DMC methods is that SMCO only considers the steady state samples. This reduces the size of the steady state gain matrix $\underline{\underline{\mathrm{A}}}$ used in the control equation, since the control and prediction horizons are both 1. For example, consider the R predictor value of a DMC single input-single output (SISO) system with a control horizon of $\mathrm{M}$ which requires $\mathrm{L}$ samples to reach steady state (Cutler, Morshedi, and Haydel, 1983):

$$
\hat{y}_{k+j}=\hat{y}_{k+j-1}+\sum_{i=0}^{M-1} a_{j-i} \Delta u_{i}
$$

where $k$ is the current sample index, $j$ is an index from 1 to $R$ where $R=L+M, \hat{y}_{k}$ is the predicted output at step $k, a_{i}$ is the response coefficient (if $i>L$ then $a_{i}=a_{L}$ ), and $\Delta \mathrm{u}_{\mathrm{k}}$ is the incremental control move at step $\mathrm{k}$ (if $\mathrm{k}<0$ then $\Delta \mathrm{u}_{\mathrm{k}}=0$ ). At any given step $k$, the $y_{k+j-1}$ term in Equation (3-13) can be treated as the output value $y_{k+j}$ if the $\Delta u_{i}$ terms are zero. To emphasize this characteristic, a superscript ${ }^{0}$ is added to the 
right-hand $\mathrm{y}$ terms. If the current step is taken as $\mathrm{k}=0$, the DMC predicted output equations from $j=1$ to $R$ then become:

$$
\begin{gathered}
\hat{y}_{1}=\hat{y}_{0}^{0}+a_{1} \Delta u_{0} \\
\hat{y}_{2}=\hat{y}_{1}^{0}+a_{2} \Delta u_{0}+a_{1} \Delta u_{1} \\
\ldots \\
\hat{y}_{M}=\hat{y}_{M-1}^{0}+a_{M} \Delta u_{0}+a_{M-1} \Delta u_{1}+\ldots+a_{1} \Delta u_{M-1} \\
\\
\ldots \\
\hat{y}_{L}=\hat{y}_{L-1}^{0}+a_{L} \Delta u_{0}+a_{L-1} \Delta u_{1}+\ldots+a_{L-M+1} \Delta u_{M-1} \\
\\
\ldots \\
\hat{y}_{R}=\hat{y}_{R-1}^{0}+a_{L} \Delta u_{0}+a_{L} \Delta u_{1}+\ldots+a_{L} \Delta u_{M-1}
\end{gathered}
$$

Now these equations can be written in a matrix and vector format:

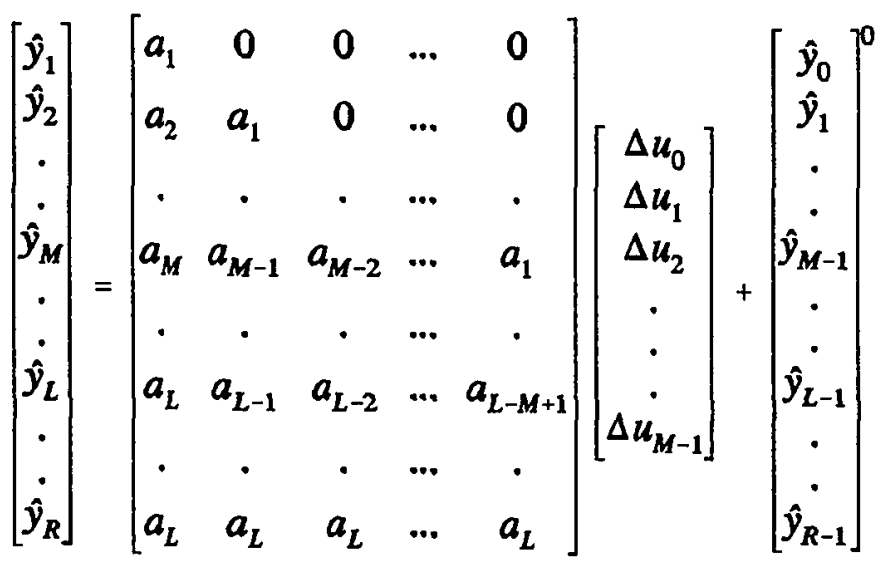


This matrix form can further be simplified to:

$$
\underline{\hat{\underline{Y}}}=\underline{\underline{A}} \Delta \underline{U}+\underline{\hat{Y}}^{0}
$$

Now note that this is the model for just one input and one output. Since SMCO is only concerned with the steady state sample or a one-step predictor, its response coefficient matrix $\underline{\underline{A}}$ reduces to a scalar term $a_{1}$. This reduction in size of the response coefficient matrix makes SMCO very appealing for control systems with limited or restricted computer resources. Note that the response coefficient matrix for DMC for a multiple input-multiple output (MIMO) is really a set submatrices, each like the $\underline{\underline{A}}$ matrix shown above. For $\mathrm{SMCO}$, the coefficient matrix consists of scalar terms like $a_{11}, a_{12}$, etc. The simpler gain matrix of SMCO also significantly reduces the effort in determining it from process identification methods.

\section{$\underline{\text { SMCO Constraints }}$}

SMCO considers only inequality constraints on the dependent variables ( $\mathrm{Y})$ since the optimization phase implies that there is some freedom of movement of the constrained variable as long as it stays above its minimum or below its maximum. If a variable must be limited to a constant value, two inequality constraints with approximately the same limits can be used to emulate an equality constraint. SMCO also places limits on the manipulated variables. The dependent variable constraints are viewed as soft constraints while those on the manipulated variables $(\mathrm{U})$ are considered hard constraints. This classification is due to the fact that the manipulated 
variables are adjusted by the algorithm and their constraints can be directly enforced. On the other hand, dependent variable constraints are really just soft boundaries that can only be enforced through the adjustment of the manipulated variables. Gain Distribution Multivariable Control (Kennedy, 1975) incorporates constraints through the error terms of its control move calculation as the upper or lower limits minus the current process values. These one-sided errors are then modified by a step penalty function, that is, the error is multiplied by zero when the constraints are not violated and by one when they are. Such a step penalty function does allow the cost to be minimized but has to correct for constraint violations after the fact. SMCO also includes constraints via its error term but defines the error as the midpoint of a constrained range minus the current output $\left(E=\mathrm{Y}^{\text {mid }}-\mathrm{Y}\right)$. This definition allows both maximum and minimum constraints to be included in a single term or the error is symmetrically distributed around the mid-point of a valid range. Like GDMC, the error term is modified by a penalty function so cost minimization can take place or the error term is not always dominating the control move calculation. After experimenting with a linear $(f=C * E)$ and cubic $\left(f=C^{*} E^{3}\right)$ penalty function, a parabolic function $\left(f=C^{*} E^{2}\right)$ is chosen for how it balances finding an optimum with taking action before constraints are violated. The constant $\mathrm{C}$ is set so that the parabolic function $f$ is unity when the variable is at its minimum or maximum constraint, that is, $C=4 /\left(Y^{\max }-Y^{\mathrm{min}}\right)^{2}$. When the variable is outside its constraints, the penalty factor $(f)$ is set to unity. A plot of the step and parabolic penalty functions is shown in Figure 3-1. The penalty factors are used to modify the 
midpoint errors $\left(\mathrm{E}_{\mathrm{i}}^{\prime}=\mathrm{f}_{\mathrm{i}}{ }^{*} \mathrm{E}_{\mathrm{i}}\right)$ used in the control move calculation. With the penalized error $E_{i}^{\prime}$, the error term is de-emphasized near its midpoint and emphasized near the constraint borders, allowing the algorithm enough freedom to find a minimum and to take action before the constraint is violated. The errors are also normalized by dividing by the constrained range $\left(Y^{\max }-Y^{\min }\right)$ before calling the control move calculation. These ideas are illustrated in the application of SMCO to a distillation process in Chapter 5.

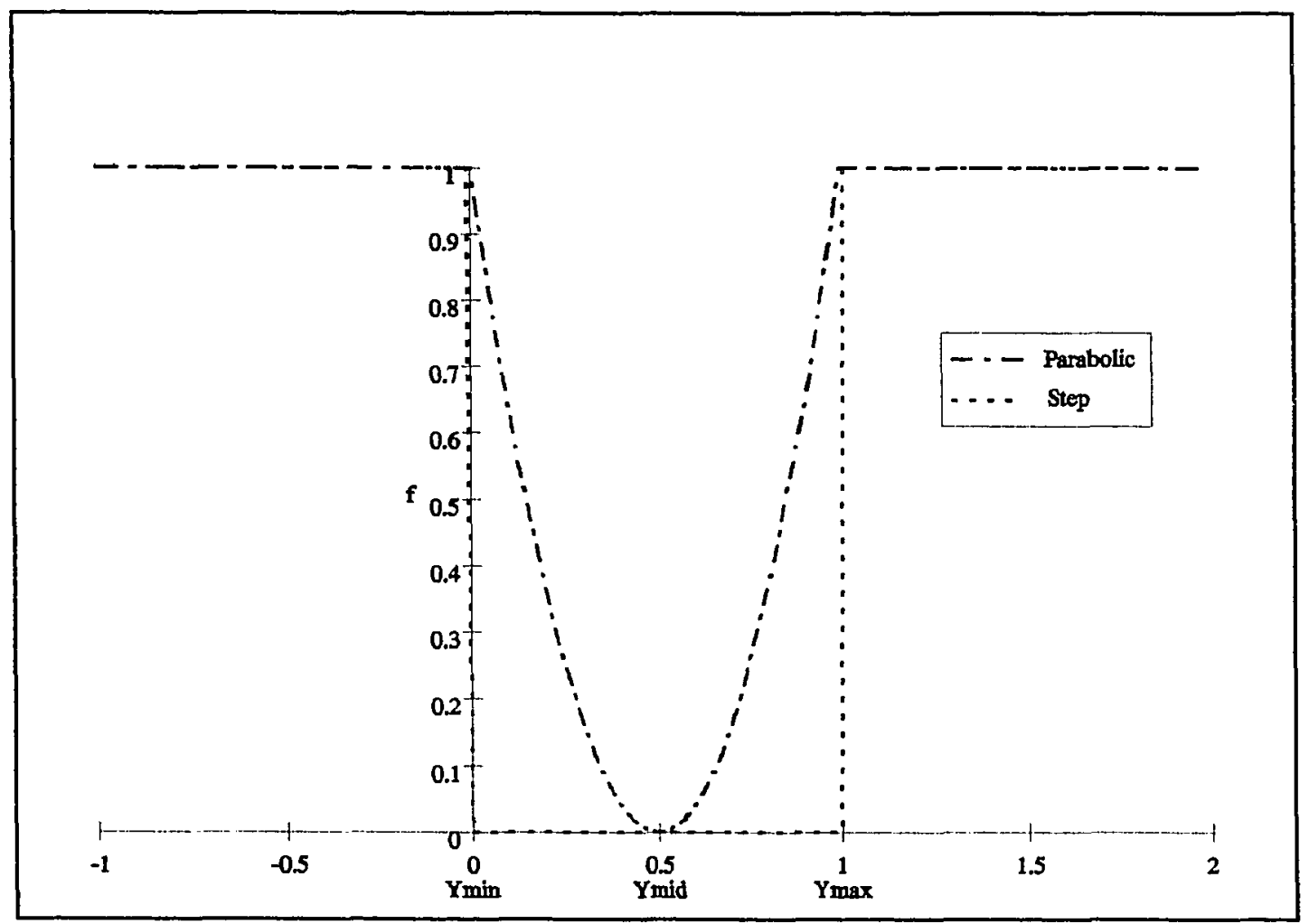

Figure 3-1. Penalty Functions for Error

Another issue to be considered is when the manipulated variables hit their hard constraints. The algorithm only gives the incremental changes to be made in the 
manipulated variables. These values must be implemented by some post processing unit, which will be discussed shortly. At this stage, any manipulated variable trying to move outside its range can be set equal to its limit. Although this method is functional, it restricts some manipulated variables to their constraints even if the optimization wants to move beyond those limits, and therefore may be preventing the remaining variables from reaching a true optimum. One solution is to still set the saturating manipulated variable to its limit but then remove it from the manipulated vector set that the algorithm considers. These ideas are incorporated into an

"extended" SMCO controller which will be discussed in Chapter 7.

\section{Implementation}

Three basic steps are needed to implement the SMCO algorithm. First, terms such as constrained errors, cost, gains, and cost partials must be calculated for the process being optimized. Second, a routine which follows Equation (3-10) must be built to take these values and evaluate the optimum moves. Finally, the optimum control moves must be processed and sent on to the low-level controllers. In an actual application on a commercial distributed control system (DCS), SMCO is implemented in three separate processing units or "tags": the first tag calculates the elements of the gain matrix $\underline{\underline{A}}$ and the cost partials from current process measurements; this tag must be custom programmed for each application. The second tag calculates the optimization moves and is general to any process. The third 
tag transmits the optimization moves to the set points of the regulatory controllers and must be configured specifically for each application.

This three stage structure has been incorporated into personal computer simulations of a distillation train and a reactor feed network to facilitate studying the algorithm. The simulations are written in an object oriented language known as Turbo Vision which is a subset of the Borland Pascal language, also known as Turbo Pascal. The SMCO algorithm is contained in one of these objects so that it may be used by other Turbo Vision applications. The SMCO procedure itself is written in a self-contained fashion where it contains the code it needs for matrix inversion so it can be ported into other languages with minor modifications. The matrix inversion is done using Gauss factorization and backward substitution procedures taken from Numerical Recipes in Fortran (1992). The Turbo Vision program which runs SMCO on the distillation train is described in Appendix B and the program for the reactor feed network is described in Appendix C. The distillation model used in these simulations is discussed in the next chapter. The reactor model will be addressed in Chapter 7.

\section{Summary}

The SMCO algorithm has been developed and compared with other methods. The constraint handling portion of the algorithm is similar to that of DMC and MAC. However, the inclusion of cost with the control move calculation is different from either of these techniques. SMCO works with inequality constraints since 
optimization implies that the variables have some freedom of movement to find the optimum. Inequality constraints can also represent equality constraints by assigning a minimum and a maximum of the same value or with a narrow range between them. A real world implementation scheme was given along with a simulation example. The model used in the distillation simulation will be discussed in the next chapter. 


\section{Chapter 4 \\ Distillation Model}

\section{Introduction}

To test the SMCO control algorithm, a program is written centered around a distillation simulation. Since the focus of this research is the development of a control algorithm and not a distillation package, a simple yet fairly accurate model is sought. At first a Fenske-Underwood-Gilliland model is used (Henley and Seader, 1981 ) but it proves inappropriate for the problem at hand. A more straight forward approach using material and energy balances is chosen instead. Both models are discussed for completeness.

\section{Fenske-Underwood-Gilliland Model}

For brevity this model is referred to as the Fenske model. It consists of the following steps (Henley and Seader, 1981):

1) Specify the split of the two key components.

2) Estimate the split of the non-key components.

3) Find column pressure and identify type of condenser.

4) Flash the feed at the column pressure.

5) Calculate minimum number of stages from the Fenske equation.

6) Calculate non-key component splits using Fenske equation and compare with estimated values. Loop back to step 3 if the values differ significantly. 
7) Calculate minimum reflux ratio from the Underwood Equation.

8) Calculate the actual number of stages for the specified reflux ratio using the Gilliland equation.

9) Calculate the feed location using the Kirkbride equation.

10) Calculate the condenser and reboiler heat duties.

To simplify the problem, the following assumptions are made. Assume that there are four components: pseudo-light, light-key, heavy-key, and pseudo-heavy. A total condenser is also assumed as well as a constant column pressure. The split of the key components is desired given the number of stages, but the Fenske model gives the number of stages for a desired split. Therefore, a loop is constructed around the model where the convergence criterion is the number of stages. The algorithm for this approach is broken down into the following steps:

1) Estimate the split of the two key components.

2) Flash the feed at the column pressure.

3) Determine distillate flow and reflux for total condenser.

4) Calculate minimum number of stages from the Fenske equation.

5) Calculate the non-key component split using the Fenske equation. If estimated and calculated values differ significantly then loop back to step 2 .

6) Calculate minimum reflux ratio from the Underwood Equation.

7) Calculate the actual number of stages for the specified reflux ratio using the Gilliland equation. If the calculated number of stages does not agree with the actual value then go back to step 1 . 
The above modified Fenske-Underwood-Gilliland model was programmed into a personal computer (PC) using the Turbo Pascal language. From preliminary test runs, the program sometimes did not converge for the specified feed location and number of stages. This behavior is not surprising since the intent of the FenskeUnderwood-Gilliland model is to only get the number of stages for a desired split and not the split for a given number of stages. Therefore, another more appropriate model had to be found.

\section{Equilibrium/Material Balance Model}

Based on the results from the Fenske model, more simplifying assumptions are made so the next model will fit better in the framework of a PC. First, the mixture is treated as a binary mix of a pseudo-light and a pseudo-heavy key component with a constant relative volatility $\left(\alpha_{\mathrm{m}}\right)$. A composition subscript of 1 refers to the heavy component while a subscript of 2 refers to the light component. The model assumes equal molar overflow and constant tray holdup in the column. The thermal condition ( $q$ ) and pseudo-heavy composition $\left(z_{1}\right)$ of the feed $(F)$ are assumed known. A diagram of a typical column used in the model is shown in Figure 4-1. The three exit streams are the bottom (B), the distillate liquid $\left(D_{1}\right)$, and the distillate vapor $\left(D_{v}\right)$. The column has a partial reboiler and a condenser, where constant heats of evaporation $\left(\mathrm{H}_{\mathrm{B}}^{\mathrm{vap}}\right)$ and condensation $\left(\mathrm{H}_{\mathrm{D}}^{\mathrm{vap}}\right)$ are assumed. The condenser can behave as a partial or total condenser depending on whether the distillate liquid or distillate vapor are preset to a zero value. The distillate condensate 


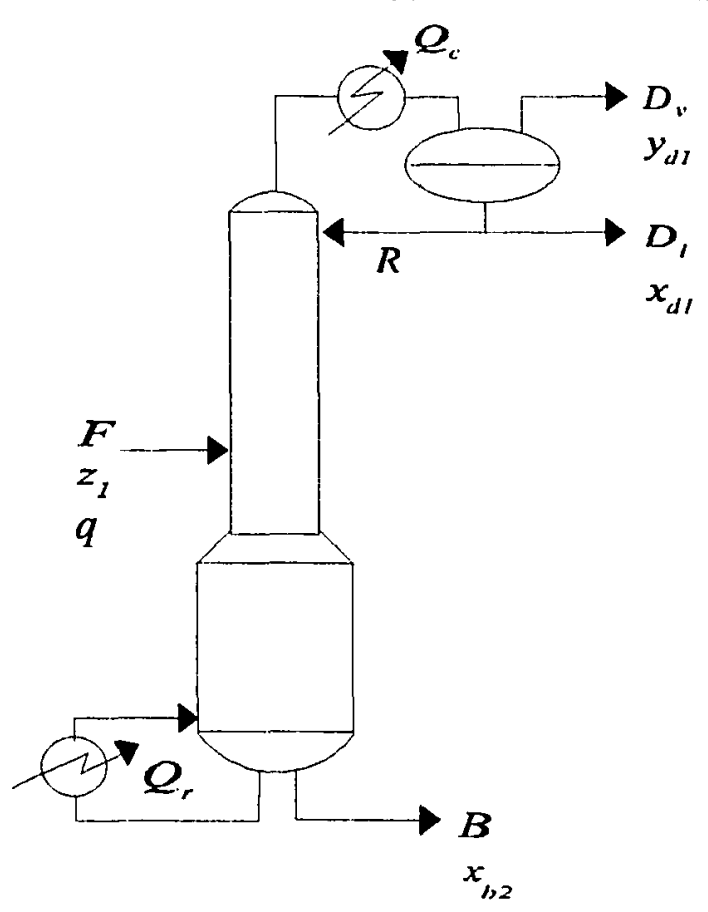

Figure 4-1. Model Distillation Column

$\left(D_{c}\right)$ splits into the reflux $(R)$ and distillate liquid $\left(D_{1}\right)$ streams. Column stages are broken into two sets. The stripping section consists of those trays below the feed including the partial reboiler $\left(\mathbf{N}_{\mathrm{s}}\right)$. The rectifying section consists of the feed tray and the trays above it including the condenser $\left(\mathbf{N}_{\mathrm{r}}\right)$. Overhead vapor or liquid and bottom impurity levels are maintained using two low-level composition controllers. The first low-level controller manipulates the reflux rate $(R)$, while the second manipulates the reboiler heat rate $\left(Q_{r}\right)$. The column reflux $(R)$ and heat rate $\left(Q_{r}\right)$ are used as the lowlevel manipulated variables based on the model equations which will be discussed shortly. Other equation sets could be used for different low-level manipulated variables, such as, condenser cooling and bottom flow. For this research, the 
reflux/reboiler heat combination is chosen because of its similarity with real applications and for its handling of partial and total condensers.

The flow equations of the distillation model are set up so given the manipulated variables of the low-level controllers $\left(Q_{r}\right.$ and $\left.R\right)$ and depending on whether the distillate liquid $\left(D_{\downarrow}\right)$ or distillate vapor $\left(D_{v}\right)$ are preset to some constant value, the other column flows can be derived. If $D_{1}$ is preset to some value, the column flows are defined by:

$$
\begin{gathered}
V_{s}=\frac{Q_{r}}{H_{B}^{\text {vap }}} \\
V_{r}=V_{s}+(1.0-q) F \\
L_{s}=q F+R \\
B=L_{s}-V_{s} \\
D_{c}=R+D_{l} \\
Q_{c}=D_{c} H_{v a p}^{D} \\
D_{v}=V_{r}-D_{c}
\end{gathered}
$$

where $V_{s}$ is the vapor flow in the stripping section, $V_{r}$ is the vapor flow in the rectifying section, $L_{s}$ is the liquid overflow for the stripping section, $D_{c}$ is the condensed distillate flow, and $\mathrm{Q}_{i}$ is the condenser cooling rate. If the distillate vapor $\left(D_{v}\right)$ is pre-set to some value then (4-5) and (4-7) are replaced by: 


$$
\begin{aligned}
& D_{c}=V_{r}-D_{v} \\
& D_{l}=D_{c}-R
\end{aligned}
$$

This pre-specification technique allows for a partial condenser if $D_{1}=0$ and a total condenser if $D_{v}=0$. There are internal checks and balances so the flows do not violate physical constraints such as total and tray mass balances.

The thermal condition of the feed, $q$, represents the energy required to bring the feed to a saturated vapor state in terms of its latent heat of vaporization:

$$
q=\frac{H_{V f}-H_{F}}{H_{V f}-H_{u f}}
$$

where $\mathrm{H}_{\mathrm{vf}}$ is the enthalpy of the saturated vapor leaving the feed tray, $\mathrm{H}_{\mathrm{Lf}}$ is the enthalpy of the liquid leaving the feed tray, and $\mathrm{H}_{\mathrm{F}}$ is the enthalpy of the feed. If $\mathbf{q}$ equals one, the feed represents a saturated liquid, and if $\mathrm{q}$ is zero, the feed is in a saturated vapor state. For values of $q$ between zero and one, the feed consists of mixed liquid and vapor phases. If $q$ is less than zero, the feed is a superheated vapor, and for values of $q$ greater than one, the feed represents a subcooled liquid. From Equation (4-2), the value of $q$ determines the maximum vapor flow $\left(F_{v}\right)$ in the column. If $q$ is less than one, the vapor flow in the rectifying section $\left(V_{r}\right)$ is greater than the vapor flow in the stripping section $\left(V_{s}\right)$ so $F_{v}$ equals $V_{r}$. When $q$ is greater than one, Equation (4-2) shows that $\mathrm{V}_{\mathrm{r}}$ is less than $\mathrm{V}_{\mathrm{g}}$ so $\mathrm{F}_{\mathrm{v}}$ becomes $\mathrm{V}_{\mathrm{g}}$. For values of $q$ equal to one, either $V_{r}$ or $V_{s}$ represent $F_{v}$ since they are equal. 
Once the column flows are calculated, the split between the pseudo-heavy and pseudo-light components can be found using Broyden's method (1969) to solve $\mathrm{N}(\mathrm{N}$ $=\mathrm{N}_{\mathrm{s}}+\mathrm{N}_{\mathrm{r}}$ ) pseudo-light component balances:

$$
\begin{gathered}
f\left(x_{i}\right)=V_{s} y_{i}+B x_{1}-L_{s} x_{i+1}=0 \quad\left(i=1 \text { to } N_{s}\right) \\
f\left(x_{i}\right)=V_{r} y_{i}+B x_{1}-R x_{i+1}-F z=0 \quad\left(i=N_{s+1} \text { to } N-1\right) \\
f\left(x_{N}\right)=F z-D_{v} y_{N}-D_{l} x_{N}-B x_{1}=0 \quad(i=N)
\end{gathered}
$$

where $x_{i}$ and $y_{i}$ represent the liquid and vapor pseudo-light component compositions in each tray $i$, respectively. These equations are the steady state pseudo-light component balances around the bottom tray and each subsequent tray in the column as shown in Figure $4-2$. Since the partial reboiler is considered the first tray, $x_{1}=x_{b 2}$ or the first tray pseudo-light composition is the same as the bottom pseudo-light composition. The condenser is considered the last tray or $\mathrm{y}_{\mathrm{N}}=\mathrm{y}_{\mathrm{d} 2}$ or overhead vapor pseudo-light composition, and $\mathrm{x}_{\mathrm{N}}=\mathrm{x}_{\mathrm{d} 2}$ or overhead liquid pseudo-light composition.

Broyden's method is generally used to solve " $n$ " nonlinear equations $(\underline{f}(\underline{x})=0)$ for " $n$ " unknowns $(\underline{x})$ by improving estimates of the inverse Jacobian. The nonlinear nature of the above equations enters in part through the vapor pseudo-light compositions $\left(y_{i}\right)$ of each tray:

$$
y_{i}=\frac{\alpha_{m} x_{i}}{1+\left(\alpha_{m}-1\right) x_{i}}
$$




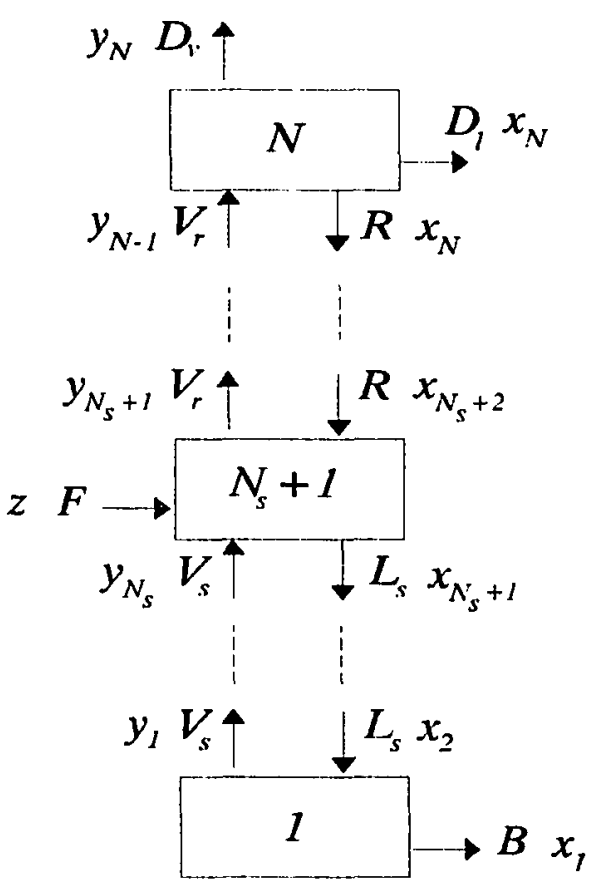

Figure 4-2. Column Trays used in Distillation Model

This equation is derived from the assumption of constant volatility for a binary mixture. The other nonlinear source comes from the low-level controllers which are included as part of the simulation by adding the following functions:

$$
\begin{aligned}
& f\left(x_{N+1}\right)=Y_{I I}-Y_{I I}^{\text {set }}=0 \\
& f\left(x_{N+2}\right)=Y_{I 2}-Y_{I 2}^{\text {set }}=0
\end{aligned}
$$

where $x_{N+1}$ and $x_{N+2}$ are the reflux $(R)$ and reboiler heat $\left(Q_{r}\right)$, respectively, and $Y_{i i}$ and $\mathrm{Y}_{\mathrm{li}}^{\text {set }}$ refer to the $\mathrm{i}^{\text {th }}$ low-level control variable and its set point, respectively. In theory, any distillation variables affected by the reflux and reboiler heat could be chosen as the low-level control variables $Y_{11}$ and $Y_{12}$. For this research, the overhead liquid $\left(\mathrm{x}_{\mathrm{d} 1}\right)$ or vapor $\left(\mathrm{y}_{\mathrm{d} 1}\right)$ pseudo-heavy composition is chosen for $\mathrm{Y}_{11}$ and the bottom 
pseudo-light content $\left(x_{b 2}\right)$ is selected for $Y_{12}$. These low-level set points become the manipulated variables of SMCO.

The distillation model consists of solving the $\mathrm{N}+2$ equations above using Broyden's method to get the pseudo-light compositions of each tray and the reflux and reboiler heat rates that force the controlled compositions to their set points. The column flows are then found using the set of equations described earlier. The convergence criterion for Broyden's method is the rate of change in the independent variables $(\underline{x})$ as adopted from Chi Yiliang (1992). To ensure proper convergence, another criterion was added where the closeness or tolerance for zero $\left(\mathrm{f}^{\text {of }}\right)$ can be specified for each function $f(x)$.

\section{Summary}

The equilibrium and material balance model was tested and proved appropriate for simulating a steady state distillation column under the given conditions. The model has been incorporated into a personal computer simulation that also includes the SMCO algorithm of Chapter 3. This simulation program is described in detail in Appendix B. The next chapter demonstrates the application of SMCO to a single distillation column and to a train of columns using the model developed here. The values of the parameters for the simulated columns are given there. 


\section{Chapter 5}

\section{Application of the SMCO Algorithm to a Distillation Process}

\section{Introduction}

This chapter examines the application of the SMCO algorithm to a single distillation column and a train of columns. The general steps for setting up SMCO for a process are discussed first and then illustrated for a distillation column. The column examined here separates out methane from heavier hydrocarbons and is called the Demethanizer column. Results are presented from a steady state simulation of this column under SMCO control. The single column application is then extended to a train of columns. The train used in this study consists of three columns to separate out ethylene from a hydrocarbon mix and is called the Ethylene Purification train. Results are given for this train using a steady state simulation. The simulation program used to generate the results for these distillation processes is described in Appendix B.

\section{Application of the SMCO Algorithm to a Process}

As the SMCO algorithm allows for an arbitrary cost function, it must be adapted to each process application. In the actual application on a commercial distributed control system (DCS), the algorithm was implemented in three separate processing units or "tags": the first tag calculates the elements of the gain matrix $\underline{\underline{A}}$ and the cost partials from current process measurements; this tag must be custom 
programmed for each application. The second tag calculates the optimization moves and is general to any process. The third tag transmits the optimization moves to the set points of the regulatory controllers and must be configured specifically for each application.

The procedure for adapting SMCO for any process consists of (1) identifying the manipulated and the constrained variables, (2) developing the gain matrix $\underline{\underline{A}},(3)$ setting up the cost function in terms of the manipulated and constrained variables, and (4) developing the partials of the cost function with respect to the manipulated variables.

The steady state gain matrix can be obtained by a number of methods. Its elements are the partials of the constrained variables with respect to the manipulated variables:

$$
A_{i j}=\frac{\partial Y_{i}}{\partial U_{j}}
$$

The individual elements can be determined from step tests of the plant, or from historical plant data, e.g., hourly averages of reactor yields or product recoveries. Some of the elements may be deduced from energy or material balance relationships involving the appropriate variables.

The most involved step of the procedure is the formulation of the cost function and the determination of the cost partials with respect to the manipulated variables. The desired result is to express the true manufacturing cost explicitly in terms of the manipulated and constrained variables. Once the cost has been molded into such a 
form, the cost partials with respect to the manipulated variables can be found. Using a model of the process, the cost differentials may be calculated in terms of measured variables. The following examples of the application to a distillation column and to a train of distillation columns will serve to demonstrate the procedure.

\section{Application to a Demethanizer Column}

A sketch of the Demethanizer column is shown in Figure 5-1. In general,

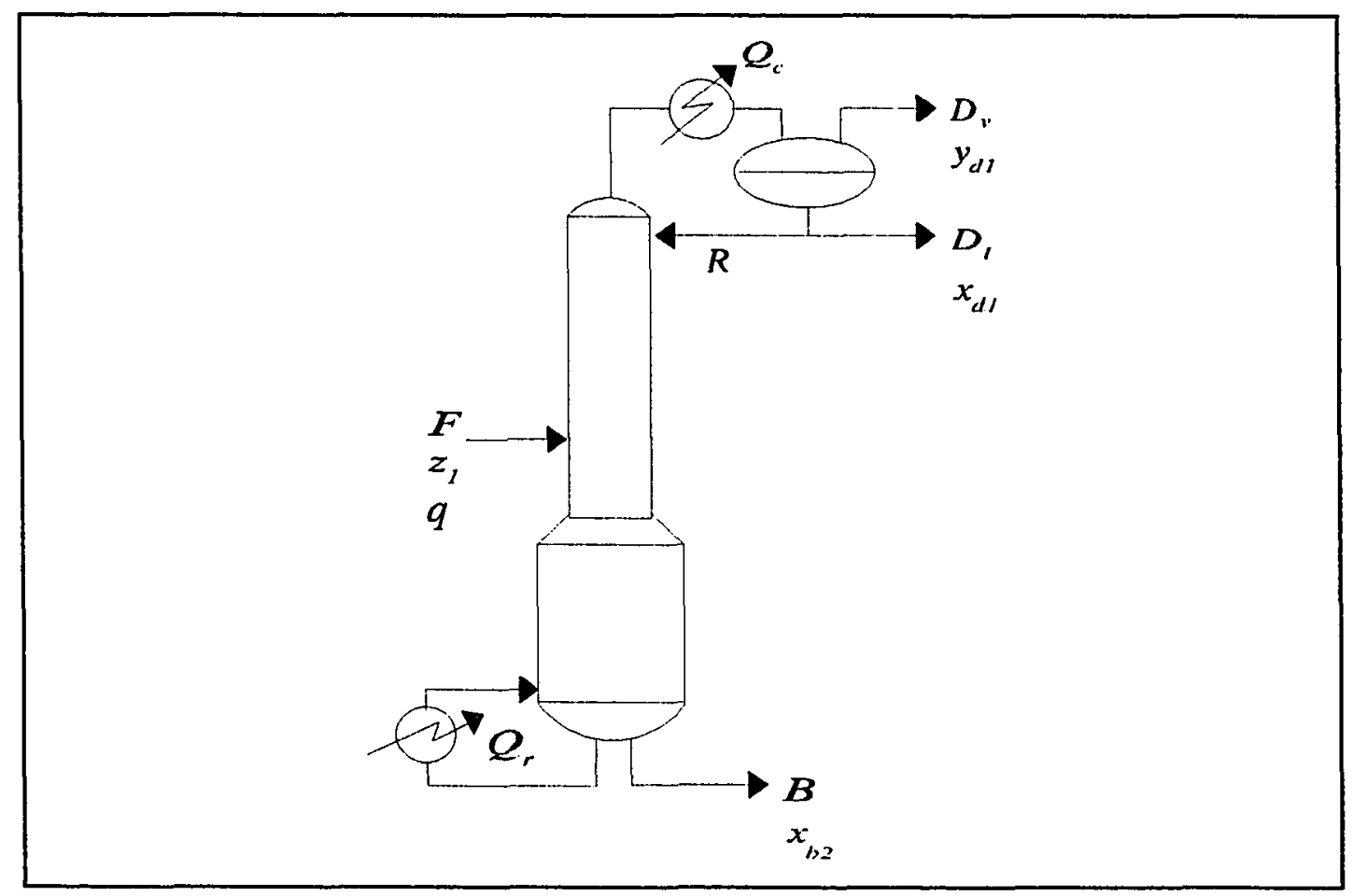

Figure 5-1. Model Distillation Column

the three exit streams are the bottom $(B)$, the distillate liquid $\left(D_{1}\right)$, and the distillate vapor $\left(D_{v}\right)$. Impurity compositions of each stream are also shown: bottom light content $\left(\mathrm{x}_{\mathrm{b} 2}\right)$, the overhead vapor heavy content $\left(\mathrm{y}_{\mathrm{d} 1}\right)$, and the overhead liquid heavy content $\left(\mathrm{x}_{\mathrm{d} 1}\right)$. The Demethanizer removes methane and lighter compounds from 
ethylene, ethane, and heavier compounds in a typical ethylene purification train. In practice there is a tight spec on the bottom methane composition $\left(\mathrm{x}_{\mathrm{b} 2}\right)$ as well as some limit on the ethylene composition in the overhead vapor product $\left(\mathrm{y}_{\mathrm{d} 1}\right)$. The overhead liquid distillate $\left(D_{1}\right)$ is usually a small purge stream in this column and may be neglected. Typical values used in the Demethanizer simulation for a feed rate of 10 klbmole/hr are shown in Table 5-i. Regulatory controllers are set up to control the two product compositions by manipulating the reboiler heat rate $\left(Q_{\mathrm{r}}\right)$ and the reflux rate (R). Flooding constitutes the main constraint and can be represented by a maximum vapor load $\left(F_{v}\right)$ on the column.

The Demethanizer optimization problem can be formulated as the minimization of the operating cost subject to vapor load flooding limit. The manipulated variables $(\underline{U})$, assuming that the feed rate is a disturbance, are the set points of the two product composition controllers, $\mathrm{y}_{\mathrm{d} 1}{ }^{\text {set }}$ and $\mathrm{x}_{\mathrm{b} 2}{ }^{\text {set }}$. In practice, the bottom light composition is maintained at a constant low value because of the tight specification on the methane composition of the bottom product, but, to illustrate how the SMCO algorithm handles a multivariable optimization, the bottom composition is allowed to vary over a range narrow enough not to affect the product value. The only dependent variable $(\underline{Y})$ for the Demethanizer is the flooding constraint, expressed as a maximum vapor rate $\left(\mathrm{F}_{\mathrm{v}}, \mathrm{klbmole} / \mathrm{hr}\right)$ in the stripping section of the column. In a real distillation column where the vapor rate is not measurable, the reflux, condenser cooling, or reboiler heat can be constrained to avoid flooding, or the column pressure drop can be measured to detect the flooding limit. 
Table 5-i. Demethanizer Simulation values with feed rate of $10 \mathrm{Klbmole} / \mathrm{hr}$

\begin{tabular}{||l|l|}
\hline Variable & Value \\
\hline \hline$N_{s}$ & 7 equilibrium stages \\
\hline \hline$N_{r}$ & 6 equilibrium stages \\
\hline \hline$\alpha_{\mathrm{m}}$ & 7.7 \\
\hline $\mathrm{z}_{\mathrm{l}}$ & 0.7 mole fraction \\
\hline \hline $\mathrm{q}$ & 1.5 \\
\hline $\mathrm{D}_{1}$ & 0 \\
\hline \hline $\mathrm{D}_{\mathrm{v}}$ & $3 \mathrm{Klbmole} / \mathrm{hr}$ \\
\hline \hline $\mathrm{y}_{\mathrm{d}}$ & $0.01 \mathrm{~mole}$ fraction \\
\hline \hline $\mathrm{R}$ & $1 \mathrm{Klbmole} / \mathrm{hr}$ \\
\hline \hline $\mathrm{B}$ & $7 \mathrm{Klbmole} / \mathrm{hr}$ \\
\hline \hline $\mathrm{X}_{\mathrm{b} 2}$ & $0.001 \mathrm{~mole}$ fraction \\
\hline $\mathrm{Q}_{\mathrm{r}}$ & $30 \mathrm{MBtu} / \mathrm{hr}$ \\
\hline \hline $\mathrm{Q}_{\mathrm{c}}$ & $2 \mathrm{MBtu} / \mathrm{hr}$ \\
\hline
\end{tabular}

The steady-state gain matrix for the Demethanizer with these manipulated and constrained variables is obtained from steps on the manipulated variables:

$F_{v}$\begin{tabular}{ll}
\multicolumn{1}{c}{$y_{d 1}^{\text {set }}$} & \multicolumn{1}{c}{$x_{b 2}^{\text {set }}$} \\
\hline-130 & -218 \\
\hline
\end{tabular}

These gains are the changes in the constrained variable per unit change in each of the manipulated variables. For example, the gain $\partial \mathrm{F}_{\mathrm{v}} / \partial \mathrm{y}_{\mathrm{d} 1}=-130 \mathrm{Klbmole} / \mathrm{hr}$ per mole fraction is the resulting change in vapor rate per unit increase in overhead product 
impurity. Increasing the impurity set point on the overhead product requires a decrease in reflux rate which eventually results in a decrease in vapor rate when the column is balanced. Similarly, the other gain is the change in vapor rate which results from a unit increase in the bottom product impurity. In this case the decrease in vapor rate results from the direct reduction in reboiler heat rate $\left(Q_{r}\right)$ by the bottom composition controller. The gains presented here were obtained from step tests on a steady-state simulation of the column.

The cost for any distillation process can be written as a sum of the material and energy streams weighted by their economic values:

$$
C=Q_{r} V_{r}+Q_{c} V_{c}+B V_{b} m_{b}+D_{v} V_{d v} m_{d v}
$$

The values $V_{i}$ represent either an income from the stream if negative, or a cost if positive. For the product streams, the extra multipliers $m_{b}$ and $m_{d v}$ are used to reflect a change in the stream value with composition. In the Demethanizer these multipliers are both unity because the purity of the distillate product does not affect its fuel value, while the impurity of the bottom product is restricted to a low value and narrow range. If the impurity of the bottom product could be set higher, the multiplier would be set as $m_{b}=1-x_{b 2}$ to reflect a drop in the value of the stream when the fraction of valuable product decreases.

Table 5-ii contains the relative 1993 values of the Demethanizer streams based on 1979 costs from Peters and Timmerhaus (1980) and from the Chemical Marketing Reporter (1993). The values are scaled from 1979 to 1993 using the Marshall and Swift indices for those years, or $952.4 / 561$. The reboiler heat value $\left(V_{r b}\right)$ is zero on 
Table 5-ii. Demethanizer Cost Values

\begin{tabular}{|c|c|c|}
\hline & 1993 Value & 1979 Base Value \\
\hline Dist. Vapor, $\mathrm{V}_{\mathrm{dv}}$ & $-\$ 60.4 / \mathrm{Klb}$ & $\$ 1.50 / 1000 \mathrm{SCF}$ \\
\hline Bottoms, $\mathrm{V}_{\mathrm{b}}$ & $-\$ 147.1 / \mathrm{Klb}$ & $*$ \\
\hline Cond. Refrig., $\mathrm{V}_{\mathrm{c}}$ & $\$ 7.07 / \mathrm{MBtu}$ & $\$ 1.20 / 288 \mathrm{KBtu}$ \\
\hline Rebr. Heat, $\mathrm{V}_{\mathrm{tb}}$ & 0 & 0 \\
\hline
\end{tabular}

*1993 value from Chemical Marketing Reporter

the assumption that the heat stream is supplied by the condensing portion of the refrigeration cycle. However, this heat source is considered uncoupled from the rate of condensation. The bottom stream value is based on the values of the components of that stream weighted by their corresponding weight fractions. The values reflect that the main product of the Demethanizer is the bottom stream, $V_{b}$, while the major cost is the condenser refrigeration rate, $V_{c}$.

The variables in the cost function of Equation (5-2) must be related to the output and manipulated variables in such a way that the dependence imposed by the over-all material, energy, and component balances is incorporated into the cost formulation. For the Demethanizer with a bottom product and a vapor distillate product these balances are:

$$
\begin{gathered}
F=D_{v}+B \\
Q_{r}+F H_{f}=B H_{b}+D_{v} H_{d v}+Q_{c} \\
y_{d l} D_{v}+\left(1-x_{b 2}\right) B=z_{1} F
\end{gathered}
$$


Note that this last equation is a balance on the pseudo-heavy component, a combination of the heavy key and heavier components. For the Demethanizer, $\mathrm{Q}_{\mathrm{r}}$ and $D_{v}$ are eliminated in terms of $Q_{c}$ and $B$ by substituting Equations (5-3) and (5-4) into Equation (5-2) to obtain:

$$
C=F\left(a_{1} P_{r}+a_{2}\right)+a_{3} Q_{c}
$$

where:

$$
\begin{aligned}
& a_{1}=V_{b} m_{b}-V_{d v} m_{d v}+V_{r b}\left(H_{b}-H_{d v}\right) \\
& a_{2}=V_{d v} m_{d v}+V_{r b}\left(H_{d v}-H_{f}\right) \\
& a_{3}=V_{c}+V_{r b}
\end{aligned}
$$

$P_{r}$ is the product recovery term which is related to the manipulated variables, $y_{d 1}$ and $x_{b 2}$, using Equations (5-3) and (5-5):

$$
P_{r}=\frac{B}{F}=\frac{z_{1}-y_{d 1}}{1-x_{b 2}-y_{d 1}}
$$

Expressing the cost function in the form of Equation (5-6) has several advantages:

first, other expressions can be used to relate the product recovery to the manipulated variables, as in Equation (5-7); second, the form can be used with other cost formulations by simply changing the expressions for the parameters $a_{1}-a_{3}$; finally, the mass, energy and component conservation restrictions are automatically incorporated into the cost function.

The next step is to develop the expression for the cost partials with respect to the manipulated variables. For the Demethanizer the manipulated variables are the set points of the two composition controllers, $y_{\mathrm{d} 1}{ }^{\text {set }}$ and $\mathrm{x}_{\mathrm{b} 2}{ }^{\text {set }}$, which, assuming that the 
regulatory controllers are operational, can be substituted for the actual compositions. From Equation (5-6) the cost partial is defined as:

$$
\frac{\partial C}{\partial u_{j}}=F a_{1} \frac{\partial P_{r}}{\partial u_{j}}+a_{3} \frac{\partial Q_{c}}{\partial u_{j}}
$$

For the Demethanizer, the product recovery partials are evaluated from Equation (5-7) as:

$$
\begin{gathered}
\frac{\partial P_{r}}{\partial u_{1}} \equiv \frac{\partial P_{r}}{\partial y_{d 1}}=-\frac{1-z_{1}-x_{b 2}}{\left(1-x_{b 2}-y_{d l}\right)^{2}} \\
\frac{\partial P_{r}}{\partial u_{2}} \equiv \frac{\partial P_{r}}{\partial x_{b 2}}=\frac{z_{1}-y_{d 1}}{\left(1-x_{b 2}-y_{d l}\right)^{2}}
\end{gathered}
$$

Notice that, for very pure products, these partials simplify, respectively, to $\left(1-z_{1}\right)$ and $z_{1}$. The $\partial Q_{c} / \partial u_{j}$ partials in Equation (5-8) can be obtained from the steady state gains through an energy balance on the condenser that relates the maximum vapor rate to the condenser heat rate:

$$
Q_{c}=H_{v a p}^{D}\left(F_{v}-D_{v}+(1-q) F\right)=H_{v a p}^{D}\left[F_{v}+F\left(P_{r}-q\right)\right]
$$

Taking partials with respect to $u_{j}$ gives:

$$
\frac{\partial Q_{c}}{\partial u_{j}}=H_{v a p}^{D}\left[A_{1 j}+F \frac{\partial P_{r}}{\partial u_{j}}\right]
$$

where we have made use of the fact that $A_{1 j}=\partial F_{v} / \partial u_{j}$. 


\section{Simulation Results}

The above application example parallels an application of the SMCO algorithm to an industrial column. To facilitate the demonstration of the algorithm performance, a simple simulation of the column was developed and programmed on a personal computer. The column model, which is described in detail in Chapter 4, assumes equal molar overflow, equilibrium stages, binary mixtures of pseudocomponents, and constant relative volatility and enthalpies. The feed rate, vapor fraction, and composition are assumed known. Broyden's method (1969) is used to solve a set of equations resulting from the component balances on each tray and the two composition controllers.

Figure 5-2 shows a three-dimensional plot of the Demethanizer cost function

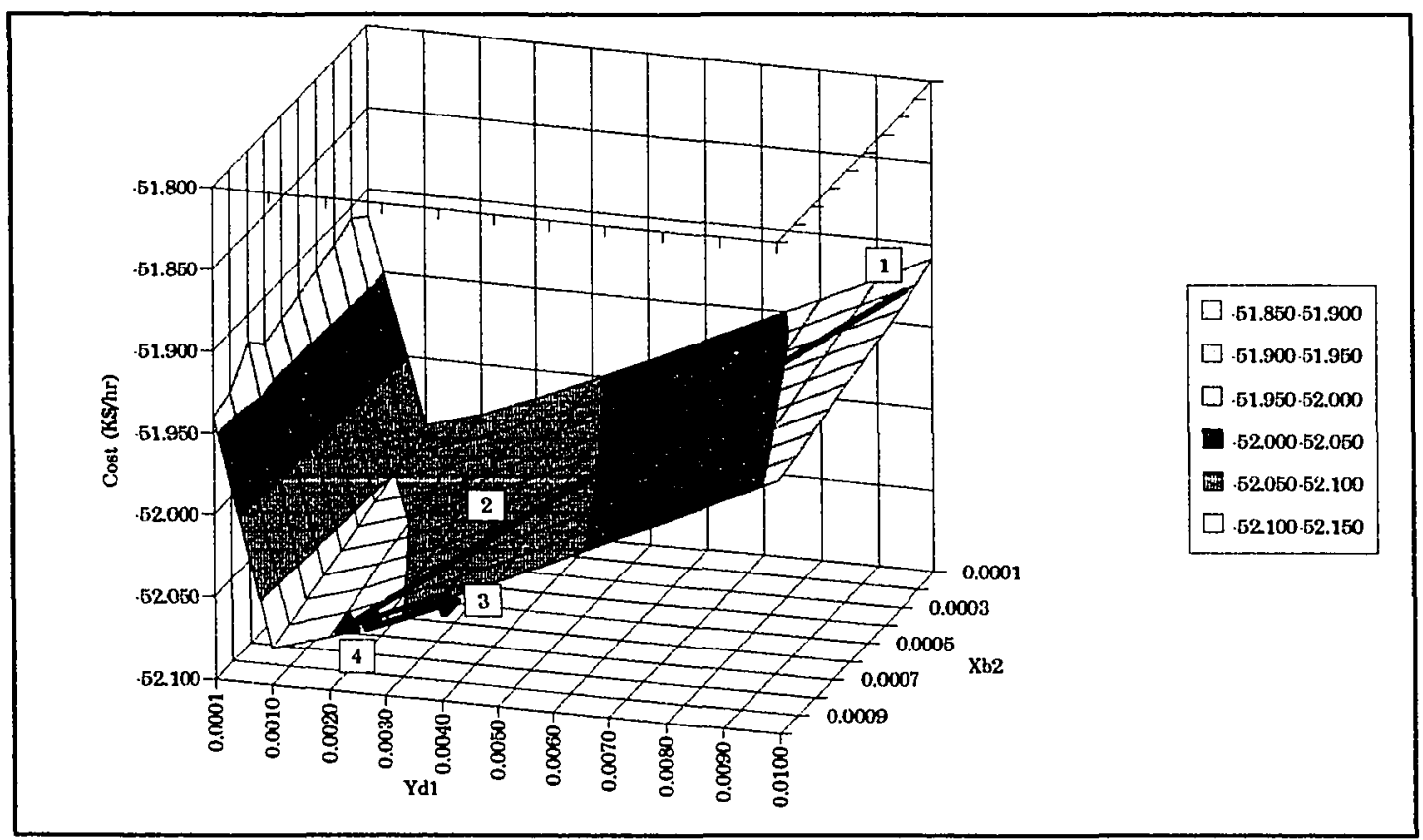

Figure 5-2. Demethanizer Cost as function of $y_{d 1}, x_{b 2}$ 
versus the manipulated variables $y_{d 1}$ and $x_{b 2}$. The path of the Demethanizer cost is outlined in Figure 5-2 by numbered arrows for the following sequence of tests: at point 1 the SMCO algorithm is initialized and it drives the cost to the optimum through the path labeled 2; a reduction in the maximum vapor rate constraint forces the cost up to point 3; finally, restoring the vapor rate limit to its initial value returns the cost to its minimum value, point 4 . The time responses of selected Demethanizer variables for the same sequence of tests are shown in Figure 5-3 where numbered arrows again show the duration of each test.

When the SMCO algorithm is turned on in stage 2, it decreases the set point of the heavies composition in the distillate $\left(\mathrm{y}_{\mathrm{d} 1}\right)$ and increases the light composition in the bottom product $\left(\mathrm{x}_{\mathrm{b} 2}\right)$, until the maximum vapor rate constraint is reached. This increases the recovery of bottom product, decreasing the cost. Note that the increase in negative cost really denotes an increase in net revenue. When the maximum vapor rate limit is decreased in test 3 , since the bottom light composition is already at its maximum, the overhead heavy composition must be increased to reduce the vapor rate. The bottom product flow then decreases because of the increase in overhead product loss, thus increasing the cost. Once the vapor rate limit is returned to its original value, the overhead composition is allowed to return to a lower value once again increasing the product recovery and lowering costs.

Notice how the constrained vapor rate comes close to its maximum limit but does not quite reach it. This behavior is a result of the parabolic penalty function for the constraint error. When the constrained variable gets a certain distance from its 

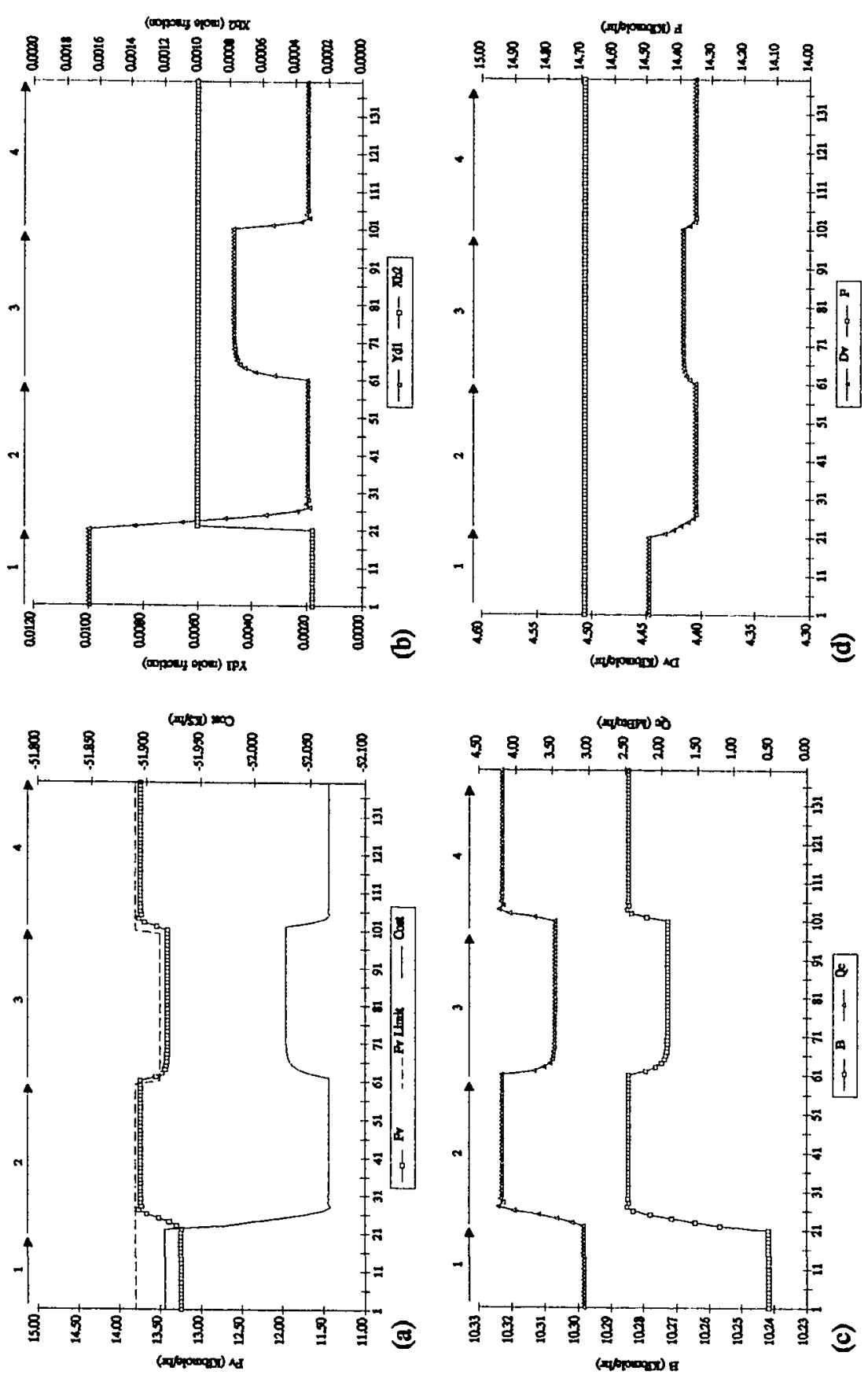

Figure 5-3. Results of SMCO Operation on Demethanizer: (a) Maximum Vapor Rate and Cost, (b) Compositions, (c) Bottom and Cooling Rates, (d) Distillate Vapor and Feed Rates 
limit, the error term dominates over the cost portion of the objective function. These results illustrate how the SMCO algorithm simultaneously satisfies operating constraints while minimizing costs for a single column.

Adaptation of the SMCO Algorithm to a Distillation Train

There are basically two modes of adapting SMCO to a train of distillation columns. One mode is similar to the one proposed by Moore and Corripio (1991) and consists of treating each column as a stage in a dynamic programming set; each column is optimized independently of the others except that the cost of each column includes the cost of the columns downstream. The other mode is to formulate the SMCO algorithm for the entire train, considering all of the manipulated and constrained variables simultaneously. The cost function is the sum of the costs of all the columns in the train and the interconnecting streams do not contribute to the cost.

In the independent mode the SMCO algorithm is applied to each column in the train, just as if it was a single column. The only difference is that the cost per unit feed of each column is calculated and passed to the upstream column as the cost coefficient for the interconnecting stream. For example, consider the ethylene purification train of Figure 5-4. The Demethanizer bottom stream value is calculated in the Deethanizer as its cost per unit feed. Similarly, the Deethanizer distillate vapor value is the cost of the $C_{2}$ Splitter divided by its feed rate.

The independent mode is appropriate when dealing with columns with significantly different steady state periods that require control moves at different time 


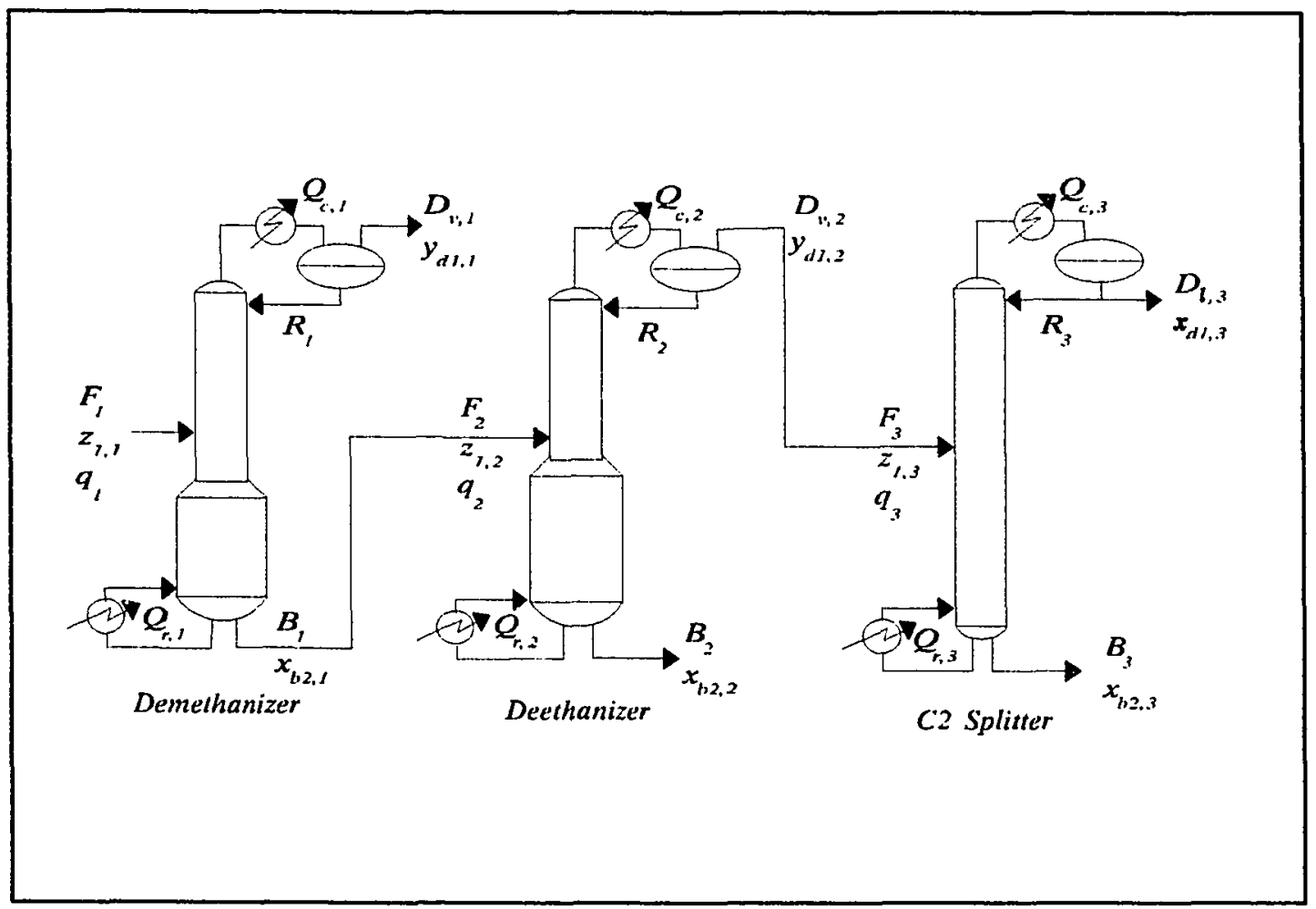

Figure 5-4. Ethylene Purification Train

intervals. A major problem with the independent mode is that, when a column is driven to its maximum capacity constraint, there is no convenient way to keep the column upstream from increasing the flow of the interconnecting stream, or to cause it to decrease the interconnecting stream flow if the constraint is already violated. This problem will be further investigated in Chapter 6 .

The application of the SMCO algorithm for the entire ethylene purification train of Figure 5-4 follows the same procedure as for the single column. Values for the product and energy streams are given in Table 5-iii and are based on 1979 costs from Peters and Timmerhaus (1980), scaled from 1979 to 1993 using the Marshall and Swift indices 952.4/561, and on chemical price data from the Chemical 
Table 5-iii. Ethylene Train Cost Values

\begin{tabular}{||c|c|c||}
\hline & 1993 Value & 1979 Base Value \\
\hline Demeth. Dist., $\mathrm{V}_{\mathrm{dv}, 1}$ & $-\$ 60.4 / \mathrm{Klb}$ & $\$ 1.50 / 1000 \mathrm{SCF}$ \\
\hline Demeth. Cond., $\mathrm{V}_{\mathrm{c}, 1}$ & $\$ 7.07 / \mathrm{MBtu}$ & $\$ 1.20 / 288 \mathrm{KBtu}$ \\
\hline Demeth. Rebr., $\mathrm{V}_{\mathrm{rb}, 1}$ & 0 & 0 \\
\hline Deeth. Bott., $\mathrm{V}_{\mathrm{b}, 2}$ & $-\$ 107.5 / \mathrm{Klb}$ & $*$ \\
\hline Deeth. Cond., $\mathrm{V}_{\mathrm{c}, 2}$ & $\$ 7.07 / \mathrm{MBtu}$ & $\$ 1.20 / 288 \mathrm{KBtu}$ \\
\hline Deeth. Rebr., $\mathrm{V}_{\mathrm{rb}, 2}$ & $\$ 3.61 / \mathrm{MBtu}$ & $\$ 2.00 / 1000 \mathrm{lb} \mathrm{steam}$ \\
\hline $\mathrm{C}_{2}$ Split. Dist., $\mathrm{V}_{\mathrm{d}, 3}$ & $-\$ 222.5 / \mathrm{Klb}$ & $*$ \\
\hline $\mathrm{C}_{2}$ Split. Bott., $\mathrm{V}_{\mathrm{b}, 3}$ & $-\$ 34.4 / \mathrm{Klb}$ & $*$ \\
\hline $\mathrm{C}_{2}$ Split. Cond., $\mathrm{V}_{\mathrm{c}, 3}$ & $\$ 7.07 / \mathrm{MBtu}$ & $\$ 1.20 / 288 \mathrm{KBtu}$ \\
\hline $\mathrm{C}_{2}$ Split. Rebr., $\mathrm{V}_{\mathrm{rb}, 3}$ & 0 & 0 \\
\hline
\end{tabular}

*1993 value from Chemical Marketing Reporter

Marketing Reporter (1993). Reboiler heat values for the Demethanizer and $C_{2}$

Splitter are zero because the heat is supplied from the condensing part of the refrigeration cycle. An additional subscript is used on the values to denote the corresponding column in the train. The most valuable product is the ethylene (distillate from column 3), and the major costs are the refrigeration loads on the condensers for each of the columns. For simplicity, the $C_{3}$ splitter column is not considered and the value of the bottom product from the Deethanizer is a weighted average of the propylene and propane in the stream. There are tight purity specifications on the bottom product compositions of the Demethanizer and Deethanizer and on the distillate composition of the $\mathrm{C}_{2}$ Splitter. Typical Deethanizer and $\mathrm{C}_{2}$ Splitter simulation values for feed rates of $10 \mathrm{klbmole} / \mathrm{hr}$ are shown in Table 5-iv. 
Table 5-iv. Deethanizer and $\mathrm{C}_{2}$ Splitter Values for feed rate of $10 \mathrm{Klbmole} / \mathrm{hr}$

\begin{tabular}{||l|l|l||}
\hline Variable & Deethanizer Value & $\mathrm{C}_{2}$ Splitter Value \\
\hline $\mathrm{N}_{\mathrm{s}}$ & 7 equilibrium stages & 9 equilibrium stages \\
\hline $\mathrm{N}_{\mathrm{r}}$ & 8 equilibrium stages & 10 equilibrium stages \\
\hline$\alpha_{\mathrm{m}}$ & 5.5 & 2.4 \\
\hline $\mathrm{z}_{1}$ & 0.2 mole fraction & 0.5 mole fraction \\
\hline $\mathrm{q}$ & 0.72 & 0.0 \\
\hline $\mathrm{D}_{1}$ & 0 & $5 \mathrm{Klbmole} / \mathrm{hr}$ \\
\hline $\mathrm{x}_{\mathrm{d} 1}$ & 0 & $0.001 \mathrm{~mole}$ fraction \\
\hline $\mathrm{D}_{\mathrm{v}}$ & $8 \mathrm{Klbmole} / \mathrm{hr}$ & 0 \\
\hline $\mathrm{y}_{\mathrm{d} 1}$ & $0.01 \mathrm{~mole}$ fraction & 0 \\
\hline$R$ & $2 \mathrm{Klbmole} / \mathrm{hr}$ & $26 \mathrm{Klbmole} / \mathrm{hr}$ \\
\hline $\mathrm{B}$ & $2 \mathrm{Klbmole} / \mathrm{hr}$ & $5 \mathrm{Klbmole} / \mathrm{hr}$ \\
\hline $\mathrm{x}_{\mathrm{b} 2}$ & $0.001 \mathrm{~mole}$ fraction & $0.01 \mathrm{~mole}$ fraction \\
\hline $\mathrm{Q}_{\mathrm{r}}$ & $50 \mathrm{MBtu} / \mathrm{hr}$ & $70 \mathrm{MBtu} / \mathrm{hr}$ \\
\hline $\mathrm{Q}_{\mathrm{c}}$ & $10 \mathrm{MBtu} / \mathrm{hr}$ & $130 \mathrm{MBtu} / \mathrm{hr}$ \\
\hline
\end{tabular}

Each column in the train is equipped with two product composition controllers and the set points of these controllers constitute the six manipulated variables. The maximum vapor load on each column constitute the three constrained variables imposed by the flooding limits. By stacking the output and manipulated variables for each column in order, the steady state gain matrices of the individual columns appear around the diagonal of the larger matrix for the train. For the train of Figure 5-4 the gain matrix is: 


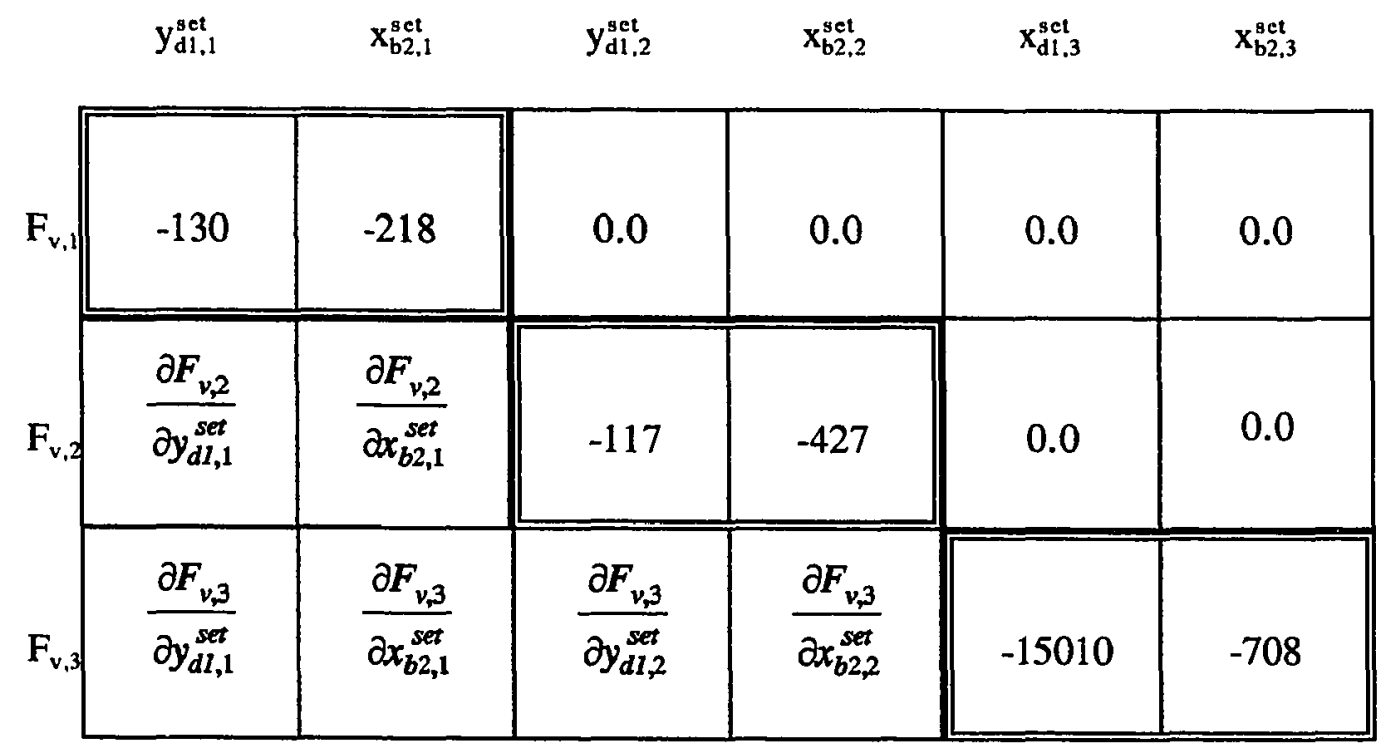

The second subscript in the variables refers to the column in the train. Individual column gain matrices have a double line around them. The gains above these matrices are zero because each column is not affected by the columns downstream, while the gains below them reflect how each column is affected by the columns upstream. These cross gains result from the effects that manipulated variables in one column have on columns downstream via the inter-connecting streams. They are obtained by expanding the partial in terms of the connecting stream. For example, consider the element $(2,1)$ of the train gain matrix above:

$$
\frac{\partial F_{v, 2}}{\partial y_{d 1,1}}=\frac{\partial F_{v, 2}}{\partial F_{2}} \frac{\partial B_{1}}{\partial y_{d 1,1}}
$$

$F_{2}$ is replaced by $B_{1}$ since the bottom product from the first column feeds the second column. The set points are replaced by their corresponding values since the controllers are assumed to function properly. Notice that all the elements needed in 
the gain matrix can be obtained from the individual columns using this concept of cross gains. These gains may be evaluated on-line using process measurements.

The next step is to build the total train cost $(C)$ which is the sum of the individual column costs:

$$
C=C_{1}+C_{2}+C_{3}
$$

$C_{1}, C_{2}$, and $C_{3}$ are the costs of the Demethanizer, Deethanizer and $C_{2}$ Splitter, respectively, and are formulated as in the single column example except the intermediate streams have values of zero $\left(V_{b, 1}=0, V_{d v, 2}=0\right)$. From the total cost formula, the cost partials with respect to each manipulated variable are obtained. Like the gains, the cost partials that represent the effect of a variable on the cost of an upstream column are zero. For example, $\partial \mathrm{C}_{1} / \partial \mathrm{y}_{\mathrm{d} 1,2}=0$ since $\mathrm{y}_{\mathrm{d} 1,2}$ does not affect the cost function for the Demethanizer. However, the terms of the cost function that are associated with an individual column will also be affected by the manipulated variables associated with the columns upstream through their effect on the rates of the connecting streams. For example, the individual column costs can be expressed as before:

$$
C_{k}=F_{k}\left(a_{1, k} P_{r, k}+a_{2, k}\right)+a_{3, k} Q_{c, k}
$$

The total cost partial with respect to $\mathrm{y}_{\mathrm{d} 1,1}$ then becomes:

$$
\begin{aligned}
\frac{\partial C}{\partial y_{d l, 1}}= & \frac{\partial C_{1}}{\partial y_{d l, 1}}+\frac{\partial F_{2}}{\partial y_{d l, 1}}\left(a_{1,2} P_{r, 2}+a_{2,2}\right)+a_{3,2} \frac{\partial Q_{c 2}}{\partial y_{d l, 1}} \\
& +\frac{\partial F_{3}}{\partial y_{d l, 1}}\left(a_{1,3} P_{r, 3}+a_{2,3}\right)+a_{3,3} \frac{\partial Q_{c 3}}{\partial y_{d l, 1}}
\end{aligned}
$$


The first term on the right of Equation (5-25) represents the regular cost partial for the Demethanizer. The other terms are the "cross" cost partials that can be evaluated using the same techniques as for the "cross" gains.

Simulation Results for Ethylene Purification Train

The optimization of the Ethylene purification train was simulated using the same sequence of tests demonstrated for the single column: the SMCO algorithm is initialized during period 1 and then allowed to minimize the cost during period 2; the maximum vapor load on the Deethanizer is reduced during period 3 and restored to its initial value during period 4. These tests are run with the SMCO algorithm operating in both the independent and the total mode. The results for both modes of operation on the Demethanizer, Deethanizer, and $\mathrm{C}_{2}$ Splitter are shown in Figure 5-5. The independent mode parameters are indicated by (I), and the total mode parameters are marked by $(\mathrm{T})$.

The Demethanizer independent cost $\left(C_{b}\right)$ and the train total cost $(C)$ both decrease when SMCO is activated as shown in Figure 5-5(a). These cost reductions are due to increases in the bottom product flow, as shown in Figure 5-5(b). The total mode cost and the independent mode cost essentially overlap for the Demethanizer since its independent cost includes the costs of the other two columns. Figure 5-5(c) shows that in both modes the Deethanizer maximum vapor rate follows its lowered limit by allowing more heavies out the top which reduces the column energy requirements and thus the vapor rates. When SMCO is activated in stage 2 , it 


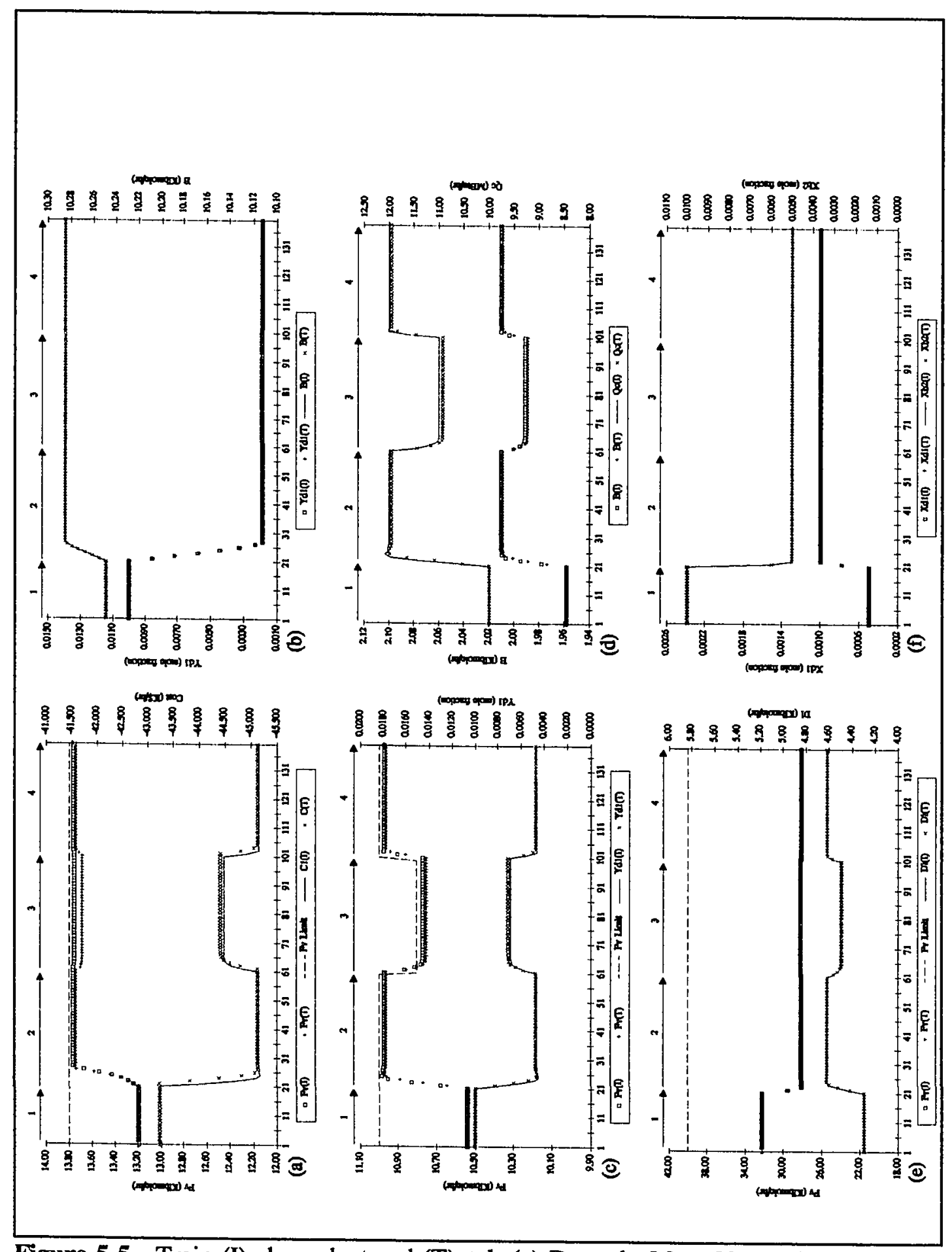

Figure 5-5. Train (I)ndependent and (T)otal: (a) Demeth. Max. Vapor \& Costs (b) Demeth. Bottoms \& Dist. Comp. (c) Deeth. Max. Vapor \& Dist. Comp., (d) Deeth. Bottoms \& Cooling, (e) $\mathrm{C}_{2}$ Split. Max. Vapor \& Dist., (f) $\mathrm{C}_{2}$ Split. Comp. 
increases the Deethanizer throughput as shown in Figure 5-5(d) until the maximum vapor constraint is approached. The profits from increased production rates overcome debits from elevated cooling to ultimately drive down costs.

The $C_{2}$ Splitter variables are shown in Figure 5-5(e) and Figure 5-5(f). When SMCO is first turned on, it drives the distillate impurity composition to its maximum and the bottom impurity composition to its minimum. The net result of these moves is a decrease in the vapor rate because it is over an order of magnitude more sensitive to the purer distillate product than to the bottom product (see the steady state gain matrix). Notice that the manipulated variables of the $\mathrm{C}_{2}$ Splitter are not affected by the changes in the vapor load limit on the Deethanizer during periods 3 and 4 . However, the ethylene product recovery is affected, showing that the initial increase in product recovery is due to the combined effect of the actions in all three columns.

\section{Summary}

The performance of SMCO in optimizing a single distillation column and a train of columns has been demonstrated. The algorithm has been implemented on a distributed control system for an industrial distillation train, and for multiple feed distribution to a group of parallel reactors. The reactor problem will be discussed in Chapter 7. By reducing the process model to a steady-state gain matrix, a compact algorithm can be created which allows for any number of constrained and manipulated variables. The user needs to decide on a cost function and then provide for the calculation of the gain matrix and cost partials for a particular application. 


\section{Chapter 6}

\section{Additional Examination of SMCO Application to Distillation Process}

\section{Introduction}

The last chapter answered many questions concerning the application of SMCO to a distillation process, but it also generated some new inquiries. For example, is there any benefit to using the independent versus total control mode when dealing with a distillation train. Another consideration is how does SMCO handle columns which do not reach steady state by the next control move. This chapter addresses these latest concerns using the same distillation process of Chapter 5 .

\section{Independent/Total Mode Difference for Ethylene Purification Train}

The last chapter shows that there are two modes of SMCO for minimizing the overall costs for a train of columns: 1) independent, which considers each column individually using a dynamic programming approach, and 2) total, where the whole train is considered as one unit. When the manipulated variables are the set points of the column product composition controllers and the constrained variables are the maximum vapor load on each column, the results from these two operational modes are very similar (see Figure 5-5). To further investigate these two control settings, the set point of the feed to the demethanizer column $\left(\mathrm{F}_{1}^{\mathrm{set}}\right)$ replaces the demethanizer bottoms composition set point $\left(\mathrm{x}_{\mathrm{b} 2,1}^{\mathrm{get}}\right)$ as the second manipulated variable. The rest of the manipulated variables and the constrained variables remain the same as in Chapter 
5. The new steady state gain matrix for the total mode then becomes:

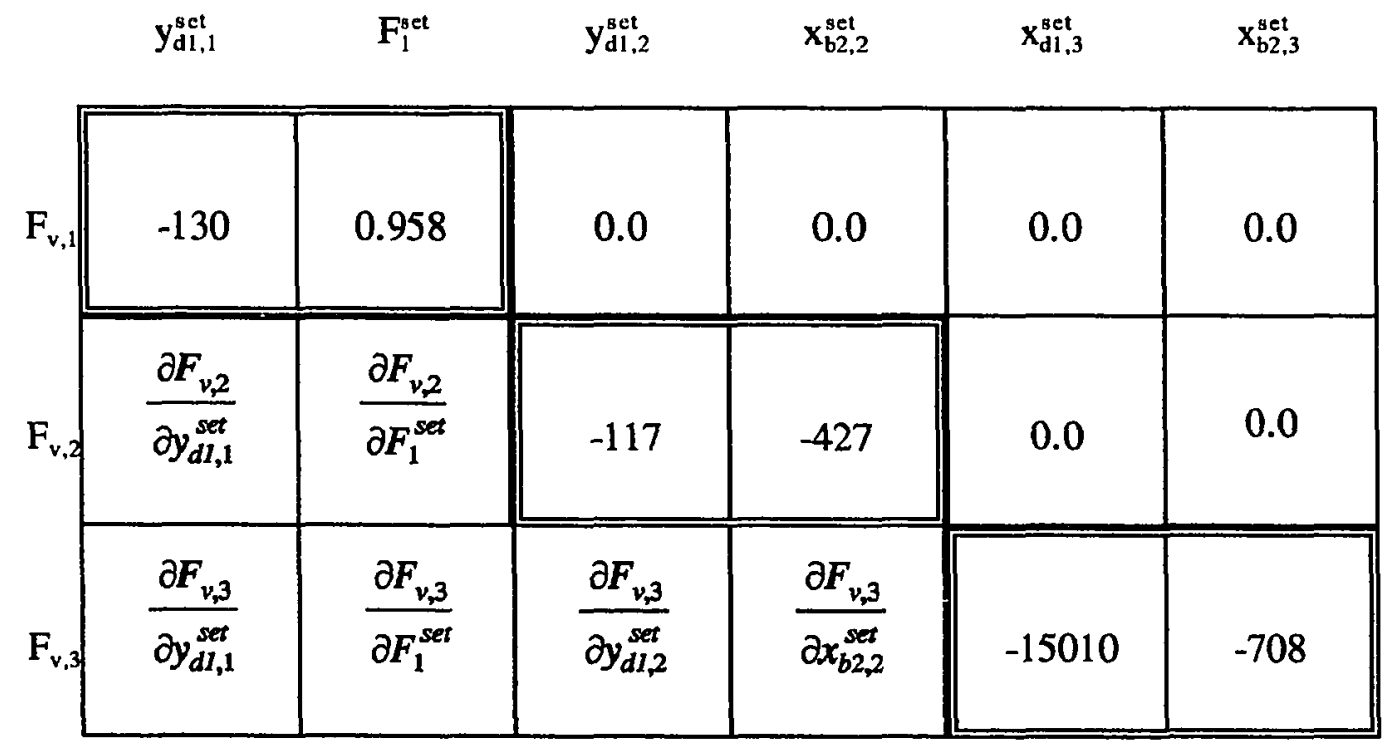

The number in the subscripts refers to the column in the train. The column gain matrices used in the independent mode have a double line around them. The gains above these matrices are zero because each column is not affected by the columns downstream, while those below them represent the "cross" gains or the effects that manipulated variables in one column have on columns downstream via the interconnecting streams. The independent mode does not consider these "cross" gains since each column is treated as one unit. The derivation of the cross gains is discussed in Chapter 5. The low-level controllers are assumed to function properly so the set points can be replaced by their corresponding process values. The same cost functions and values defined in Chapter 5 are used here. The cost partials are derived the same way except the identity of one of the manipulated variables has changed. 
Simulation Results with Manipulated Feed to Train

The runs for the Ethylene Purification Train in Figure 5-5 are repeated where: the SMCO algorithm is initialized during period 1 and then allowed to minimize the cost during period 2; the maximum vapor load on the Deethanizer is lowered during period 3 and restored to its initial value during period 4 . These tests are run with the SMCO algorithm operating in both the independent and total modes. The results for both modes of operation are shown in Figure 6-1. The independent mode parameters are indicated by (I), and the total mode parameters are marked by (T).

When the algorithm is turned on, the independent and total costs both decrease at about the same rate and to the same final value as shown in Figure 6-1(a) by increasing the feed to the train as illustrated in Figure 6-1(b). Similar results are observed in Figure 5-5. However, when the maximum vapor limit is lowered during run 3 , the independent operation does not bring the variable below its constraint since the manipulated variables of the Deethanizer are saturated at their limits as presented in Figure 6-1(c). The only way to drive the maximum vapor down would be to increase $y_{d 1}$ which is already at its upper limit or to decrease the bottom composition $\left(x_{b 2}\right)$ which is at its lower limit. However, the total mode is able to make the Deethanizer maximum vapor follow its constraint by decreasing the feed to train or the Demethanizer feed as shown in Figure 6-1(a). This result is due to the fact that the total mode considers how each column affects those downstream from it (via the cross gains in the train gain matrix) and can take action accordingly. The total cost increases during the third run since less feed into the train means less product out, 


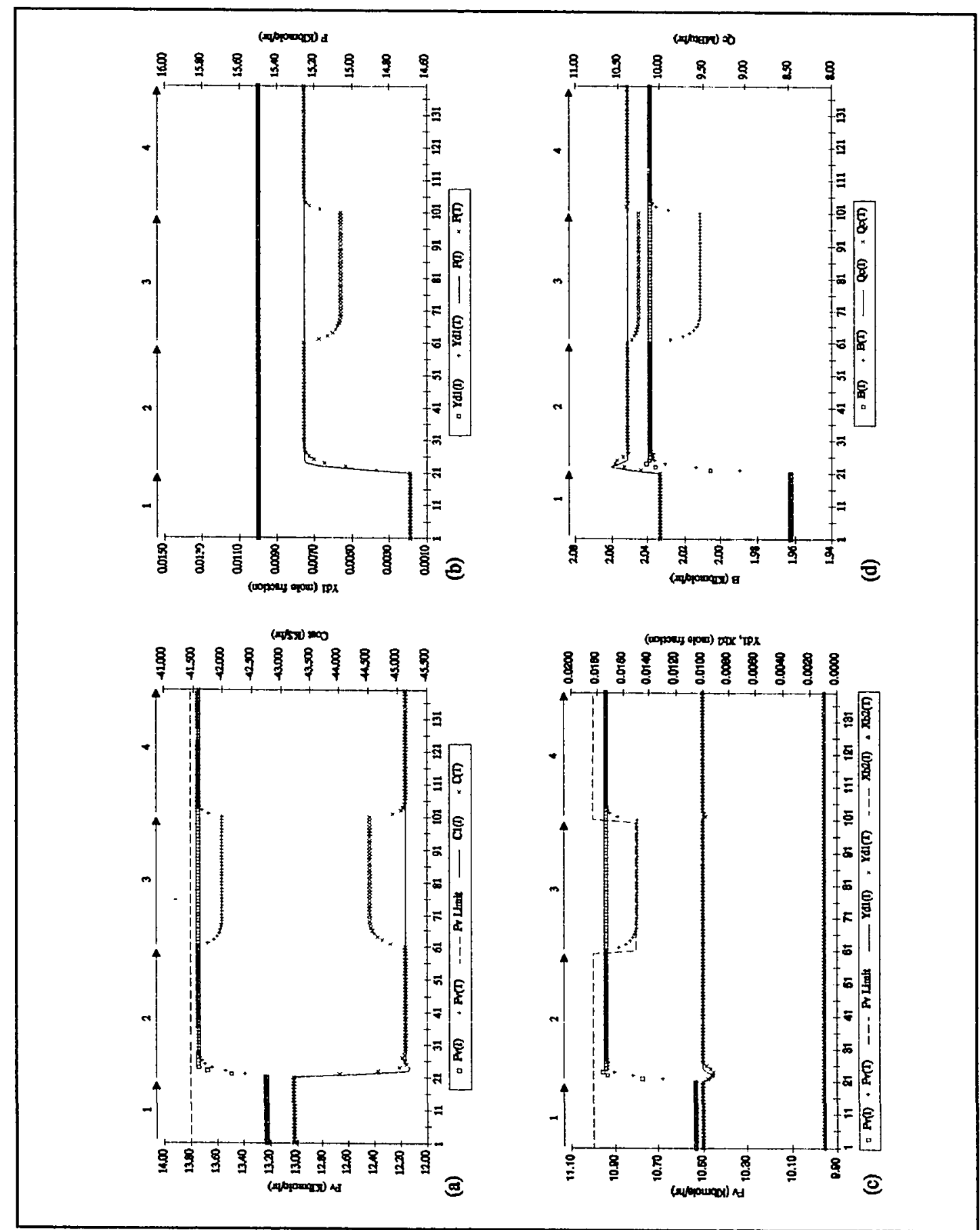

Figure 6-1. (I)ndependent and (T)otal Results with Manip. Feed: (a) Demeth. Maximum Vapor and Costs, (b) Demeth. Overhead Comp. and Bottoms, (c) Deeth. Maximum Vapor and Overhead Comp., (d) Deeth. Bottoms and Cooling Rates 
but the algorithm is designed to satisfy constraints first before considering costs. When the Deethanizer maximum vapor limit is returned to its original value, the costs from the independent and total modes again overlap. The $C_{2}$ Splitter behaves as it did in Chapter 5 (see Figure 5-5) where ethylene production is maximized by reducing the lights out the bottom $\left(\mathrm{x}_{\mathrm{b} 2}\right.$ driven to its lower limit) and by allowing more heavies out the top ( $x_{\mathrm{d} 1}$ driven to its upper limit).

Based on this last set of runs, the total mode is preferable to the independent mode when the manipulated variables of one column are saturating but columns upstream may have some maneuvering capability. The total mode considers the effect of each column on downstream processes so if some constraint is violated downstream and cannot be resolved by that column's controllers, then the upstream column can take action.

\section{Columns not Reaching Steady State between Updates}

The next topic to be investigated is whether SMCO can drive a process, such as the distillation train, to its optimum when one or more units, columns in this case, are not reaching steady state between the control updates. To examine this problem the same set-up as described in Chapter 5 for the Ethylene Purification Train is used, where the manipulated variables are the overhead and bottom impurity set points and the maximum vapor is being constrained. Note for this scenario that the independent mode performs as well as the total mode since no control moves are saturating when a constrained variable reaches its limit. In theory, the independent mode could be 
used for a system where the individual units do not reach steady state at the same interval or the control moves have to be updated at different intervals. In the independent mode, each column manipulates only its controllers using the latest information it has sampled. Whether this sampled data is behind or ahead of another column is not critical because each instance of SMCO has been tuned for its particular column so constraints should always be satisfied. Now for the total mode, the assumption is made that all columns have reached steady state by the next control move. When the individual columns have different settling times then the longest time must be chosen for the total control interval. Even with this choice the total mode may not make appropriate moves since one or more columns could be in between steady states. In the same manner, on-line gains and cost partials evaluated in the pre-processing stage of the algorithm may be off since a column is not at steady state.

To examine these issues of columns not reaching their steady states, the PC program is modified to lag all model variables which are normally sent directly to the SMCO control algorithm. The on-line gains and cost partials in the pre-processing stage are also calculated using the lagged model variables. Note that any variables which represent set points for low-level controllers are not lagged. The transfer function for a first order lag between output $\mathrm{Y}^{\mathrm{fil}}(\mathrm{s})$ and input $\mathrm{Y}(\mathrm{s})$ can be expressed as:

$$
\frac{Y^{f i l}(s)}{Y(s)}=\frac{K}{\tau s+1}
$$


where $\mathrm{K}$ is the gain and $\tau$ is the time constant of the lag. Taking the Z-transform of this expression with zero-order hold (Corripio, 1982) and assuming a sample interval of $T$ gives:

$$
Y_{i}^{f l}=\left(1-q_{f i l}\right) Y_{i-1}+q_{f i l} Y_{i-1}^{f l}
$$

where $K$ is assumed to be unity, the $i$ and $i-1$ subscripts refer to the current and previous samples, respectively, and $\mathrm{q}_{\mathrm{fil}}$ is defined as $\mathrm{e}^{-\mathrm{T} / \tau}$. This first order lag can be approximated by an exponential filter or:

$$
Y_{i}^{f l}=\left(1-q_{f l}\right) Y_{i}+q_{f i l} Y_{i-1}^{f i l}
$$

where $\mathrm{Y}_{\mathrm{i}}^{\mathrm{fil}}$ is the filtered value of the current process value $\mathrm{Y}_{\mathrm{i}}, \mathrm{Y}_{\mathrm{i}-1}^{\mathrm{fll}}$ is the previously filtered value, and $q_{i j 1}$ is the filter factor. These filtered values can be treated as the lagged values. Using this technique, any model variables normally used in evaluating the on-line gains, costs, and error terms are replaced by their filtered or lagged counter parts. In this manner, the optimization sees values which do not represent the current steady state values of the system.

To emulate columns not reaching steady state between control moves in the simulation, filter factors are chosen as $q_{f i l}=0.06,0.12$, and 0.5 for the Demethanizer, Deethanizer, and $\mathrm{C}_{2}$ Splitter, respectively. The same runs as outlined in the first part of this chapter are then executed using the same weights as in Chapter 5 where columns were assumed to reach steady state between control samples. The Demethanizer and Deethanizer results for both modes are shown in Figure 6-2(a) and 
(c). As before the cost is minimized in both modes but using the non-steady state data in the Deethanizer causes the maximum vapor to slightly overshoot its upper limit as shown in Figure 6-2(c). After adjusting only the move suppression parameters for the Deethanizer and repeating the runs, the maximum vapor stays below its upper limit as shown in Figure 6-2(d) while giving approximately the same cost savings as seen in Figure 6-2(c). These last runs do show that when SMCO sees the columns in a train as not reaching steady state, some constraint violation may occur but can be corrected by adjusting the move suppression parameters. This fine tuning is only necessary when the constraint riding its limit belongs to a column where the steady state values are significantly off like in the Deethanizer. Since the maximum vapor for the $C_{2}$ Splitter never gets near its minimum, the lagged or nonsteady state values pose no problems. In fact, the $\mathrm{C}_{2}$ Splitter results are not shown because they are virtually identical to those in Figure 5-5. The independent and total results for the Demethanizer are basically the same as in Chapter 5 since the filtering is small or the lagged variables are very close to their true steady state values. Even for the Deethanizer, the results are very similar to those in Chapter 5 mostly due to the relatively small filter factor. Based on these findings another set of runs is made with a higher filter factor just on the Deethanizer to examine the effects.

The runs for Figure 6-2 are repeated with only the Deethanizer not reaching steady state between control updates. For this setup the filter factors for the Demethanizer, Deethanizer, and $\mathrm{C}_{2}$ Splitter columns are $0,0.5$, and 0 , respectively. To establish a base case for comparison the same set of runs and weights as used in 


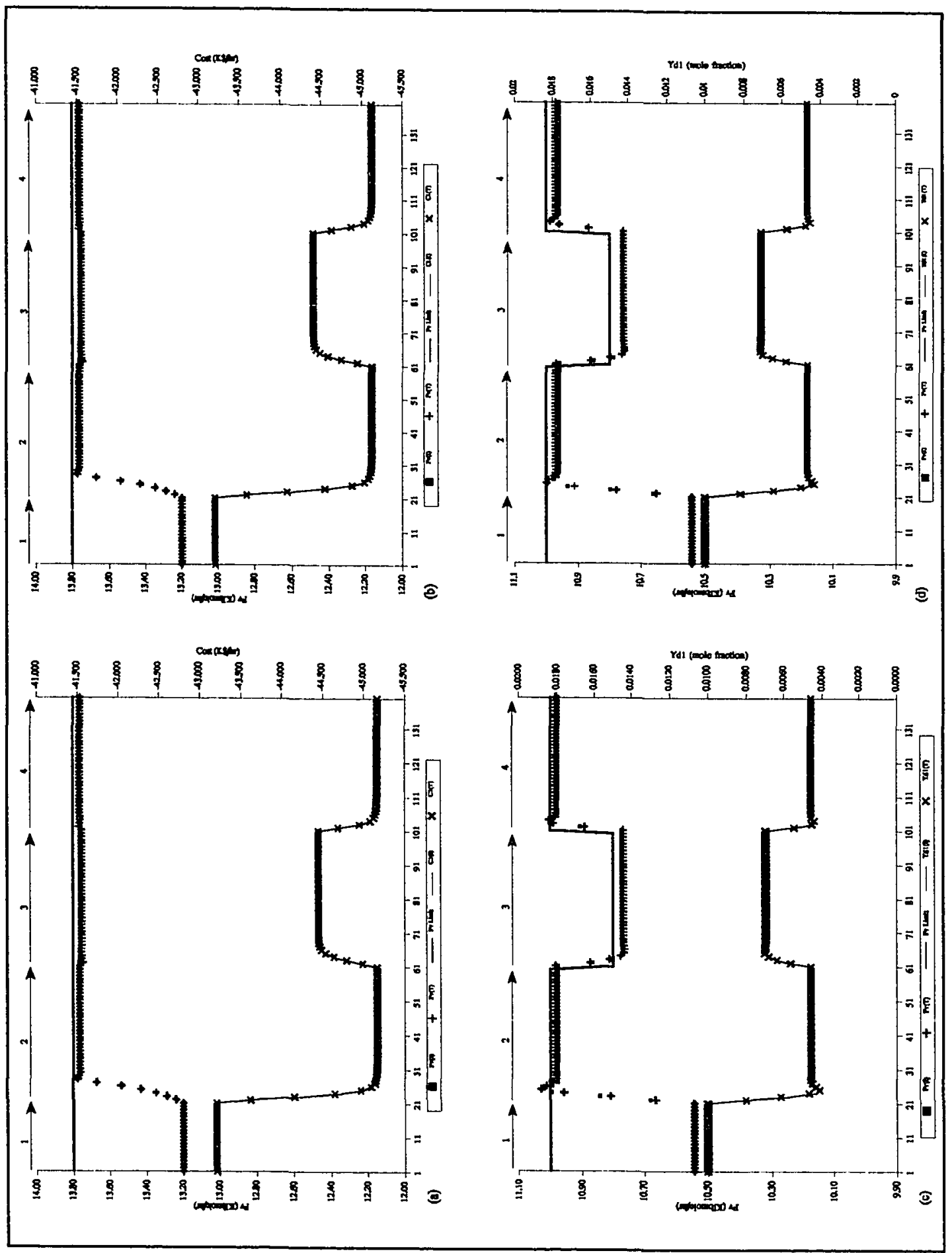

Figure 6-2. (I)ndependent and (T)otal Results with three uneven settling times: (a) Demeth. Maximum Vapor and Costs, (b) Demeth. Overhead Comp. and Bottoms, (c) Deeth. Maximum Vapor and Overhead Comp., (d) Deeth. Bottoms and Cooling Rates 
Chapter 5 are again used but with the new set of filter factors. This base case is shown in Figure 6-3(a) and (c). The response of the Demethanizer is practically the same as seen in Figure 6-2 but the Deethanizer has a much larger overshoot of its constrained vapor rate due to its heavily lagged variables. Even with a large discrepancy in the Deethanizer steady state values, the cost is still minimized. To correct for the overshoot in the Deethanizer, its move suppression parameter is increased and the results are shown in Figure 6-3(d). The cost still drops but not as far as before since the Deethanizer flooding limit is now being followed. The $\mathrm{C}_{2}$ Splitter still behaves as shown in Figure 5-5 with the maximum vapor staying well above its lower limit. These results show that even with a large offset in the steady state values which is passed to the on-line gain estimates, costs, and error terms, SMCO behaves fairly robustly and can be tuned to correct for any constraint violations.

\section{Summary}

From these additional runs on the distillation train, it has been shown that the total mode of SMCO may be beneficial if a column constraint changes and the manipulated variables of that column are already saturated. The total mode may be able to alter the manipulated variables of a column upstream to satisfy the new constraint. If some columns in a train are not reaching steady state by the next optimization sample and their constrained variables are close to their limits then there might be some constraint violations. If a constraint violation occurs, then the move suppression parameter of the affected column can be increased to avoid such 


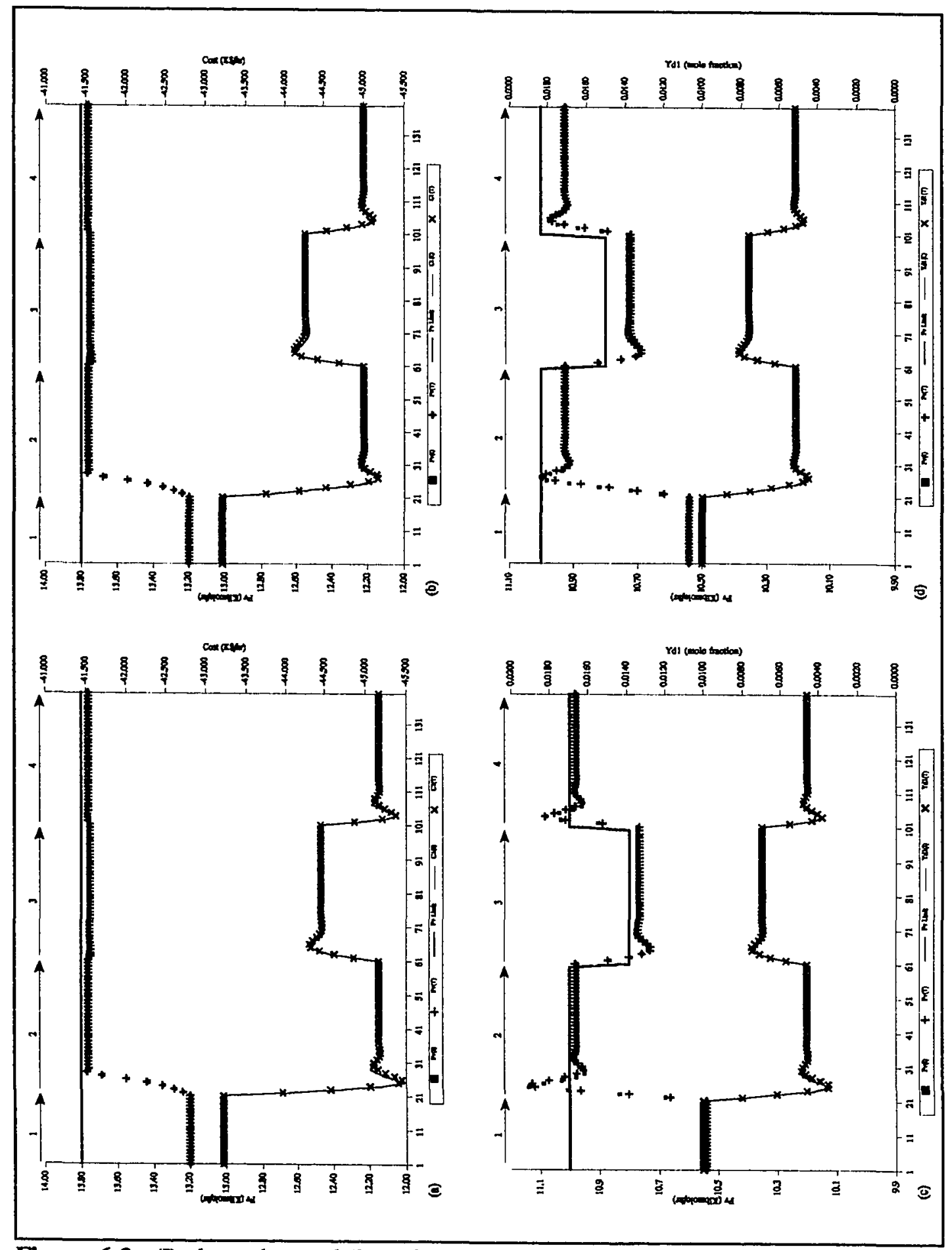

Figure 6-3. (I)ndependent and (T)otal Results for one uneven settling time: (a) Demeth. Maximum Vapor and Costs, (b) Demeth. Overhead Comp. and Bottoms, (c) Deeth. Maximum Vapor and Overhead Comp., (d) Deeth. Bottoms and Cooling Rates 
overshoots. If the problem is more of columns having different settling times than not reaching steady state, the independent mode should be used for the reasons discussed earlier. In the next chapter, the SMCO algorithm is applied to a set of parallel reactors to determine the best feed rate to each reactor. 


\section{Chapter 7}

\section{Application of the SMCO Algorithm to Optimize Feed Distribution to Reactors in Parallel}

Introduction

This chapter examines an application of the Supervisory Multivariable Constrained Optimization algorithm that finds the optimum feed rates to a set of parallel reactors to minimize the production costs while satisfying feed supply and product demand constraints. There are common raw feed stocks $\left(R_{k}\right)$ that are drawn upon to feed the reactors as shown in Figure 7-1. The breakdown of a reactor feed $\left(F_{j}\right)$ is specified by a term $B_{k j}$ which tells the amount of $k^{\text {th }}$ raw feed $\left(R_{k}\right)$ that goes into a pound of the reactor feed. The reactor network supplies product streams for downstream processes where total production rates $\left(P_{i}\right)$ are monitored and constrained. The raw feed rates are also limited based on inventory. The goal of SMCO is to find the optimum settings for the reactor feeds that will reduce the production costs while satisfying the current constraints.

This chapter also addresses the issue of manipulated variables hitting hard constraints. The SMCO algorithm is designed to calculate the incremental changes in the manipulated variables. These values are then passed to a post processing unit that carries out the changes making sure the hard constraints are not violated. This method restricts the manipulated variables to their limits even if the optimization wants to move beyond those boundaries, and therefore may be preventing the other moves from 


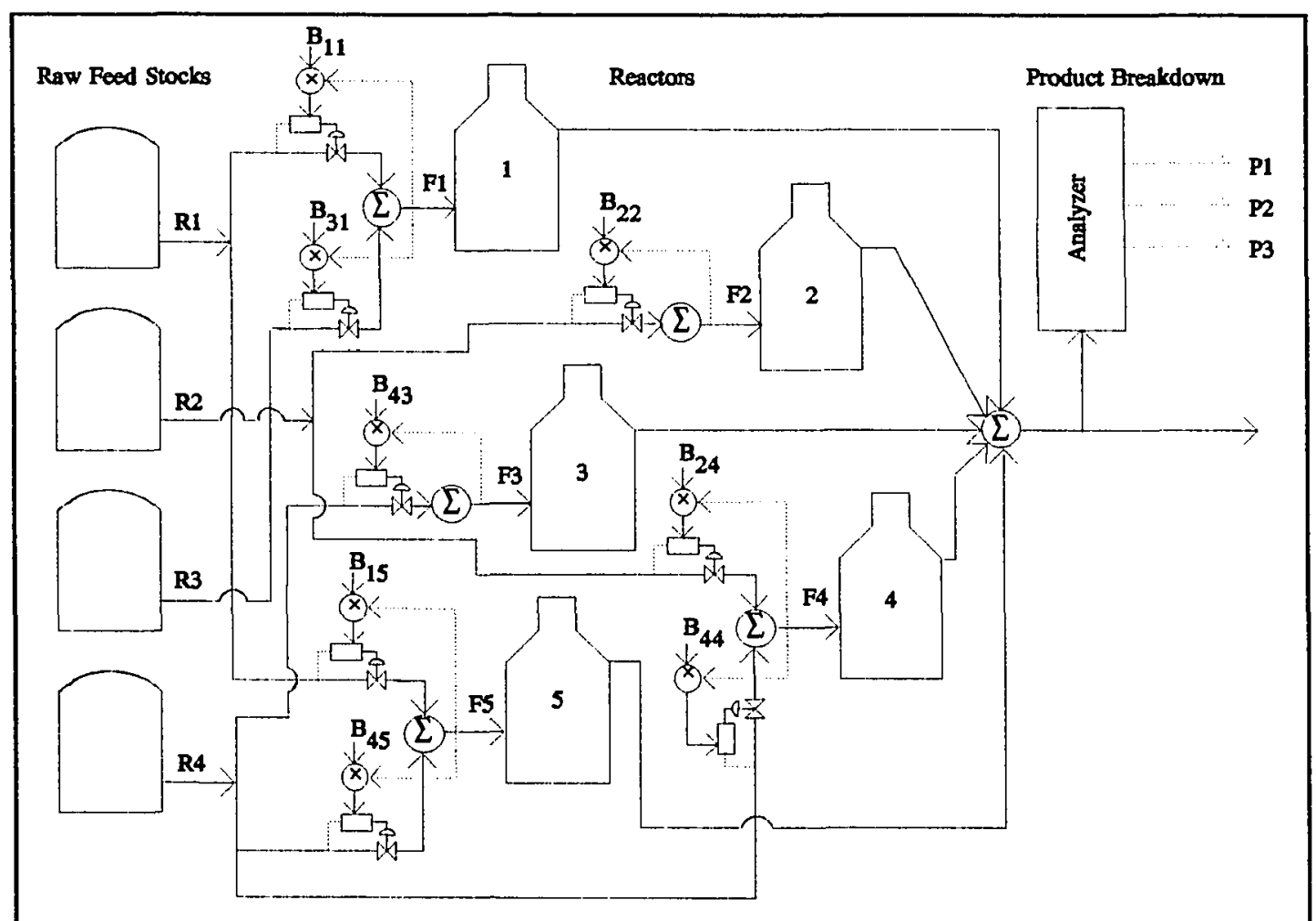

Figure 7-1. Distributed Feed Reactor Network showing partial connection of raw feeds.

reaching the lowest cost. To remedy this problem, a manipulated variable that is pushed up against its constraint is set equal to its limit and then removed from the manipulated vector set that the algorithm considers. At each iteration the algorithm considers all manipulated variables even those that may have been previously dropped in case the control moves bring the variable back within its valid range. These ideas are incorporated into an "extended" controller where flags are set that show if a manipulated variable is available in the post processing phase. A demonstration of this enhanced controller is given for the parallel reactors. 


\section{Application of the SMCO Algorithm to Reactor Feed Distribution}

\section{Reactor Model}

To model the reactor network, two gain matrices are used. The first matrix $\underline{\underline{B}}$ is given in Table 7-i and shows the optimum breakdown of each reactor feed in terms of the mass fraction of raw feeds. For example, the $\mathrm{k}^{\text {th }}$ row and $\mathrm{j}^{\text {th }}$ column element of $\underline{\underline{B}}\left(B_{k j}\right)$ indicates the pounds of $k^{\text {th }}$ raw feed $\left(R_{k}\right)$ that make up a pound of $j^{\text {th }}$ reactor

Table 7-i. Feed Breakdown Matrix $\underline{\underline{B}}$ for Reactors

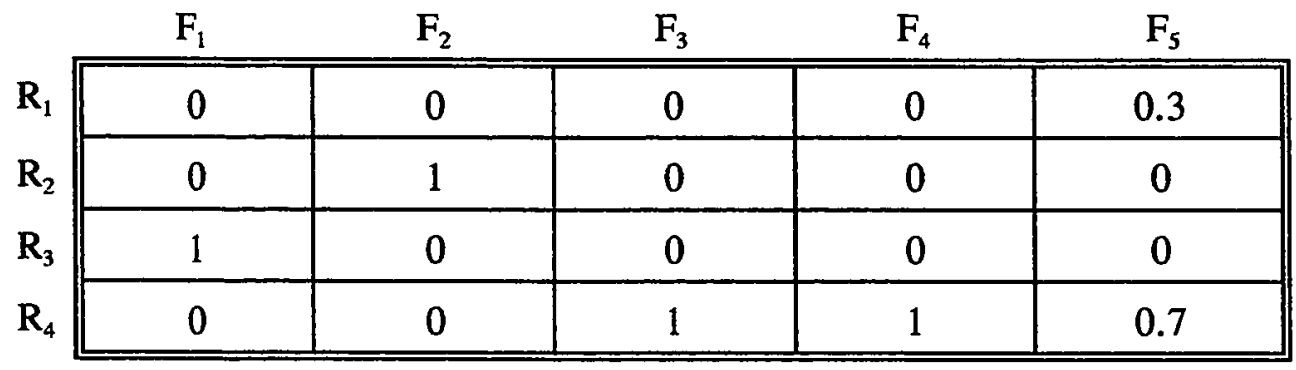

feed $\left(F_{j}\right)$. Consequently, the columns of $\underline{\underline{B}}$ must sum to one. The $\underline{\underline{B}}$ matrix can also be viewed as the optimum allocation of raw feeds to the reactor network. For example, the first column of $\underline{\underline{B}}$ shows that all feed to reactor one $\left(F_{1}\right)$ comes from the third raw feed stock $\left(\mathrm{R}_{3}\right)$. This allocation assumes that the reactor operating conditions, such as temperature and pressure, are maintained at values which produce optimum output. As pointed out by Ramsey (1990), plant-wide integrated optimization can be broken into two stages: off-line and on-line. The off-line optimization occurs less often (daily, weekly, etc) as the market values, operation 
schedules, or supply lines change. The on-line optimization deals with the current operation of the process and is updated frequently (hourly). Determination of $\underline{\underline{B}}$ can be considered part of the off-line optimization and should be updated as conditions warrant. Using this feed allocation matrix, the following steady state model for the raw feeds can be written:

$$
R_{k}=\sum_{j=1}^{M} B_{k j} F_{j}
$$

where $R_{k}$ represent the mass flow rates of the raw feed stocks and $F_{j}$ are the total feed rates to each reactor.

The second gain matrix $\underline{Q}$ in the reactor model gives the product yields in terms of the raw feeds and is shown in Table 7-ii. Each $i^{\text {th }}$ row and $\mathbf{k}^{\text {th }}$ column element $\left(Q_{i k}\right)$ gives the pounds of $i^{\text {th }}$ product $\left(P_{i}\right)$ manufactured per pound of $k^{\text {th }}$ raw feed $\left(R_{k}\right)$ sent to the reactor network. Note that $\underline{Q}$ only contains the yield data for

Table 7-ii. Yield Matrix $\underline{Q}$ for Reactor Products

\begin{tabular}{|c|c|c|c|}
\hline $\mathbf{R}_{1}$ & $\mathbf{R}_{2}$ & $\mathbf{R}_{3}$ & $\mathbf{R}_{4}$ \\
\hline 0.15 & 0.07 & 0.15 & 0.08 \\
\hline 0.49 & 0.54 & 0.22 & 0.17 \\
\hline 0.04 & 0.02 & 0.19 & 0.13 \\
\hline
\end{tabular}

products being constrained, i.e., the columns of $\underline{\underline{Q}}$ may or may not sum to one depending on whether all the products are being constrained. These product yields assume that the reactors operating conditions, such as temperature and pressure, are 
maintained at values to produce optimum yields. Determination of $\underline{Q}$ can also be considered part of the off-line optimization. When there is a major operating change in the reactors or product levels begin to fluctuate, the elements of $Q$ should be reevaluated. $\underline{\underline{Q}}$ can be determined by averaging past production data of the reactors. Using this matrix, the steady state model for the products is written as:

$$
P_{i}=\sum_{k=1}^{N} Q_{i k} R_{k}
$$

where $P_{i}$ represents the mass flow rates of the product streams and $R_{k}$ are the raw feed rates. The on-line optimization is handled by the SMCO algorithm which is described next.

\section{SMCO Application}

The procedure for applying SMCO to any process consists of:

1) identifying the manipulated $(\underline{U})$ and the constrained $(\underline{Y})$ variables,

2) developing the gain matrix $\underline{\underline{A}}$,

3) setting up the cost function in terms of the manipulated and constrained variables,

4) developing the partials of the cost function with respect to the manipulated variables.

The constrained variables in the reactor feed problem consist of the raw feed flow rates $\left(R_{k}\right)$ and the product flow rates $\left(P_{i}\right)$. There are four raw feed stocks labeled as $R_{1}, \quad R_{2}, R_{3}$, and $R_{4}$ and three product streams identified as $P_{1}, P_{2}$, and $P_{3}$. The 
constrained vector used by SMCO is $\underline{Y}=\left[R_{1}, R_{2}, R_{3}, R_{4}, P_{1}, P_{2}, P_{3}\right]^{T}$. The manipulated variables are the total feeds to each reactor $\left(\mathrm{F}_{\mathrm{i}}\right)$. There are five reactors, and so the manipulated vector is constructed as $\underline{\mathrm{U}}=\left[\mathrm{F}_{1}, \mathrm{~F}_{2}, \mathrm{~F}_{3}, \mathrm{~F}_{4}, \mathrm{~F}_{5}\right]^{\mathrm{T}}$.

The steady state gain matrix $\underline{\underline{A}}$ used by SMCO represents the changes in the constrained variables per unit change in the manipulated variables. For the reactor network the constrained vector is $\underline{Y}=\left[R_{1}, R_{2}, R_{3}, R_{4}, P_{1}, P_{2}, P_{3}\right]^{T}$ and the manipulated vector is $\underline{U}=\left[F_{1}, F_{2}, F_{3}, F_{4}, F_{5}\right]^{T}$. The raw feed model of Equation (7-1) fits into this form so the upper four rows of the steady state gain matrix $\underline{\underline{A}}$ are the same as matrix $\underline{\underline{B}}$. The product model in Equation (7-2) must be transformed using Equation (7-1):

$$
P_{i}=\sum_{k=1}^{N} \sum_{j=1}^{M} Q_{i k} B_{k j} F_{j}
$$

From this last equation it is seen that the product of the $\underline{Q}$ and $\underline{\underline{B}}$ matrices relates a change in the reactor feed rates to the product streams. Therefore, the last three rows in the steady state gain matrix $\underline{\underline{\mathrm{A}}}$ are the same as the product of matrices $\underline{Q}$ and B. The $\underline{\underline{A}}$ matrix for this reactor system is shown in Table 7-iii where the partition between the $\underline{Q}$ matrix and the product matrix $\underline{\mathrm{QB}}$ is represented by a dotted line. It should be noted that the $\underline{\underline{A}}^{\mathrm{T}} \underline{\underline{W}}^{\mathrm{T}} \underline{\underline{\mathrm{WA}}}$ matrix in Equation (3-10) evaluated for unity weights $(\underline{\underline{W}}=\underline{I})$ has two dependent columns and so non-zero move suppression parameters must be used to make the columns independent and the matrix invertible.

The cost $C$ to be minimized in the reactors is the sum of the raw feeds $\left(R_{k}\right)$ times their cost per pound $\left(\mathrm{V}_{\mathrm{rk}}\right)$ and the products $\left(\mathrm{P}_{\mathrm{i}}\right)$ times their value per pound 
Table 7-iii. Steady State Gain Matrix $\underline{\underline{A}}$

\begin{tabular}{|c|c|c|c|c|c|}
\hline & $F_{1}$ & $\mathrm{~F}_{2}$ & $\mathrm{~F}_{3}$ & $\mathrm{~F}_{4}$ & $\mathrm{~F}_{5}$ \\
\hline $\mathbf{R}_{1}$ & 0 & 0 & 0 & 0 & 0.3 \\
\hline $\mathrm{R}_{2}$ & 0 & 1 & 0 & 0 & 0 \\
\hline $\mathbf{R}_{\mathbf{3}}$ & 1 & 0 & 0 & 0 & 0 \\
\hline $\mathbf{R}_{4}$ & 0 & 0 & 1 & 1 & 0.7 \\
\hline$P_{1}$ & 0.15 & 0.07 & 0.08 & 0.08 & 0.10 \\
\hline$P_{2}$ & 0.22 & 0.54 & 0.17 & 0.17 & 0.27 \\
\hline$P_{3}$ & 0.19 & 0.02 & 0.13 & 0.13 & 0.10 \\
\hline
\end{tabular}

$\left(V_{p}\right)$ : Fuel costs per pound of raw feed are assumed equal for all reactors and are not included. Substituting Equation (7-2) for the product term $\mathbf{P}_{i}$ gives:

$$
\begin{gathered}
C=\sum_{k=1}^{4} R_{k} V_{r k}+\sum_{i=1}^{3} P_{i} V_{p i} \\
C=\sum_{k=1}^{4} R_{k} V_{r k}+\sum_{i=1}^{3}\left(\sum_{k=1}^{4} Q_{i k} R_{k}\right) V_{p i}
\end{gathered}
$$

The raw feed term $\left(R_{k}\right)$ can be factored out to give:

$$
C=\sum_{k=1}^{4}\left(V_{r k}+\sum_{i=1}^{3} Q_{i k} V_{p i}\right) R_{k}=\sum_{k=1}^{4} C_{k} R_{k}
$$

where $C_{k}$ can be viewed as the operating cost per pound of raw feed. Negative values represent a profit while positive values represent a debit in these cost equations. The product and raw feed values used in the simulation are shown in 
Table 7-iv. These values are based on costs from Peters and Timmerhaus (1980) and from the Chemical Marketing Reporter (1993). The values from Peters and Timmerhaus are scaled from 1979 to 1993 using the Marshall and Swift indices for those years, or 952.4/561 (Chemical Engineering, 1993). The raw feed stocks and products are assigned values based on the 1993 manufactured gas cost of $\$ 2.55 / 1000$ scf and the 1993 fuel value of $\$ 2.51 / \mathrm{MBtu}$. The other product values are taken from Chemical Marketing Reporter (1993).

Table 7-iv. Reactor Cost Values

\begin{tabular}{|c|c|}
\hline Stream Values & 1993 Value \\
\hline $\mathrm{V}_{\mathrm{r} 1}$ & $\$ 27.1 / \mathrm{Klb}$ \\
\hline $\mathrm{V}_{\mathrm{r} 2}$ & $\$ 32.3 / \mathrm{Klb}$ \\
\hline $\mathrm{V}_{\mathrm{r} 3}$ & $\$ 3.73 / \mathrm{Klb}$ \\
\hline $\mathrm{V}_{\mathrm{r} 4}$ & $\$ 3.73 / \mathrm{Klb}$ \\
\hline $\mathrm{V}_{\mathrm{p} 1}$ & $-\$ 60.4 / \mathrm{Klb}$ \\
\hline $\mathrm{V}_{\mathrm{p} 2}$ & $-\$ 222.5 / \mathrm{Klb}$ \\
\hline $\mathrm{V}_{\mathrm{p} 3}$ & $-\$ 127.5 / \mathrm{Klb}$ \\
\hline
\end{tabular}

To evaluate the cost partials needed in the SMCO algorithm, the cost equation (7-6) must be put in terms of the manipulated variables $F_{i}$. To accomplish this task, the raw feed model of Equation (7-1) with $M=5$ (five reactor feeds) is substituted into Equation (7-6) to give the cost in terms of the manipulated variables:

$$
C=\sum_{k=1}^{4}\left[\sum_{j=1}^{5} B_{k j} F_{j}\right] C_{k}
$$

Now the cost partials with respect to the manipulated variables $F_{j}$ can be defined as: 


$$
\frac{\partial C}{\partial F_{j}}=\sum_{k=1}^{4} B_{k j} C_{k}
$$

With the formulation of the reactor costs as in Equation (7-6), the products can all be assigned a zero value. SMCO will then find feed flow rates that minimize the production costs. When non-zero product values are used, the algorithm will select flow rates that maximize profits. These aspects of the cost formula are demonstrated in the results.

\section{Simulation Results}

A personal computer simulation incorporating the reactor network and the SMCO algorithm is used to study various aspects of the feed distribution problem. This simulation is discussed in Appendix C. Figure 7-2 shows the normalized raw feed and product flows along with material cost $\mathrm{C}$ for the following set of runs:

1) SMCO in manual,

2) optimization turned on,

3) the second product maximum $\left(\mathrm{P}_{2}^{\max }\right)$ is lowered,

4) $P_{2}^{\max }$ is returned to its original value.

Note that flows are normalized so 0 corresponds to the lower constraint and 1 corresponds to the upper constraint of each variable. For these runs the cost values of Table 7-iv are used. The product values are non-zero, and so the algorithm will maximize profits. During run 1 , the flows are at their nominal operating values. Turning SMCO on in step two causes the material cost $C$ to drop as more $P_{1}, P_{2}$, and 


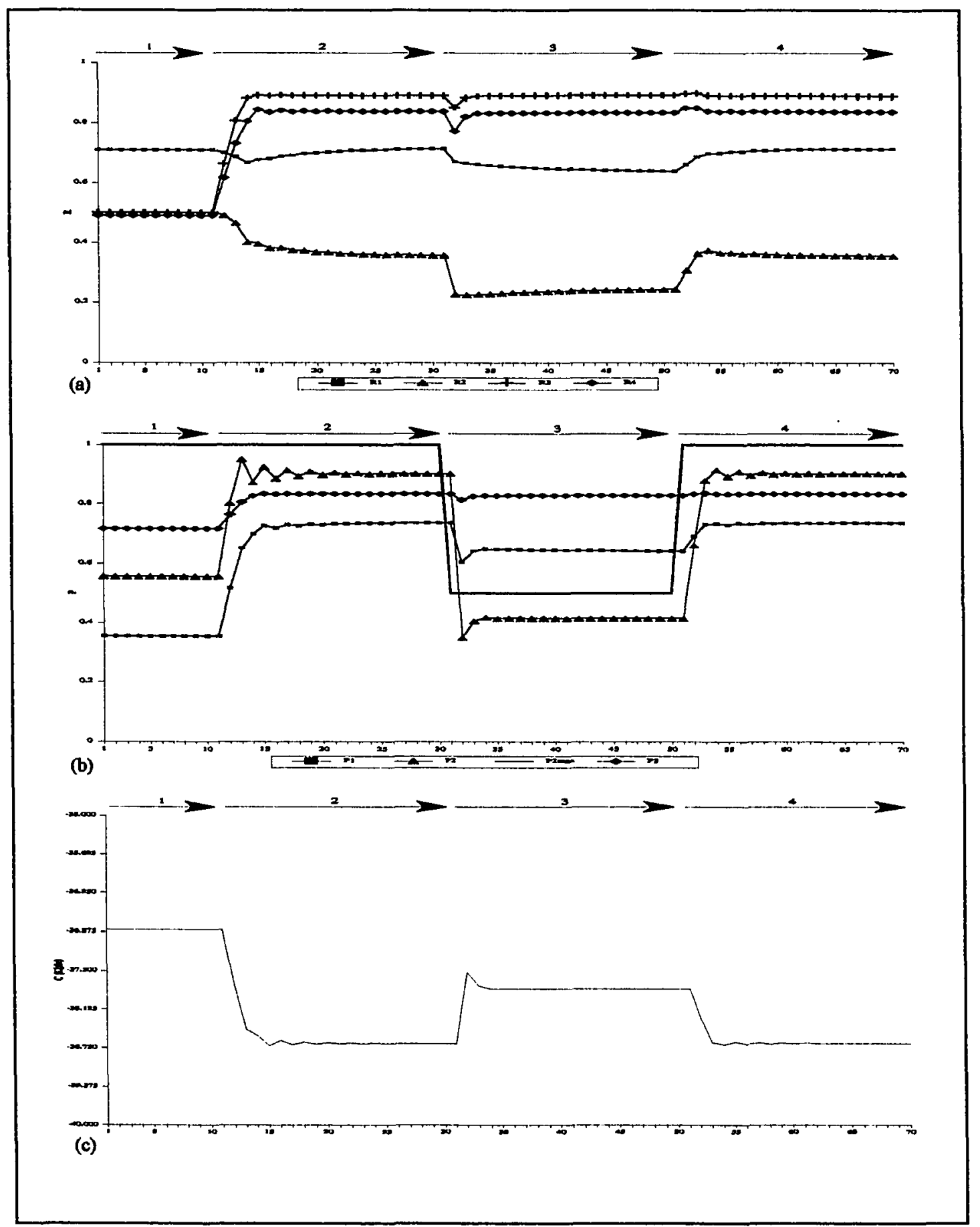

Figure 7-2. Parallel Reactor Results for Maximizing Profits: (a) Normalized Raw Feeds, (b) Normalized Product Streams, (c) Costs 
$P_{3}$ are produced using less of the most expensive raw feed $\left(R_{2}\right)$ and more of the inexpensive ones $\left(R_{3}, R_{4}\right)$ until $P_{2}$ and $R_{3}$ near their upper limits. The second most expensive raw feed rate $\left(R_{1}\right)$ dips down initially to reduce costs but returns to its original value to make up production loss from the dropping $R_{2}$ flow rate. Note that negatively decreasing cost is analogous to positively increasing profit. The cost levels out as product $P_{2}$ and raw feed $R_{3}$ near their upper limits due to the nature of the parabolic penalty function chosen for the constraint errors. The algorithm is designed so constraint violations take precedence over cost minimization as shown in the third run where the forced suppression of product $P_{2}$ causes the cost to jump up since it is the most valuable product. Raising the production limit during the fourth run causes the cost to drop again until the $P_{2}$ and $R_{3}$ values get close enough to their limits for the impending constraint violations to overcome cost reduction.

To evaluate how well the algorithm minimizes production costs, a similar set of runs is executed as above except the product values are all assigned a value of zero and the second product minimum $\left(\mathrm{P}_{2}{ }^{\mathrm{min}}\right)$ is raised and lowered during steps three and four. Figure 7-3 shows the normalized raw feed and product flows for these tests. As expected when SMCO is turned on during the second run, costs are lowered by decreasing the usage of the most expensive raw feeds $\left(R_{1}, R_{2}\right)$ and increasing the consumption of the least expensive raw feeds $\left(R_{3}, R_{4}\right)$. It may seem more logical to decrease all raw material demand since products have no assigned economic value. However, the algorithm is taking into account the constraints on minimum production levels, in particular, the minimum level for product $\mathrm{P}_{2}$. To 


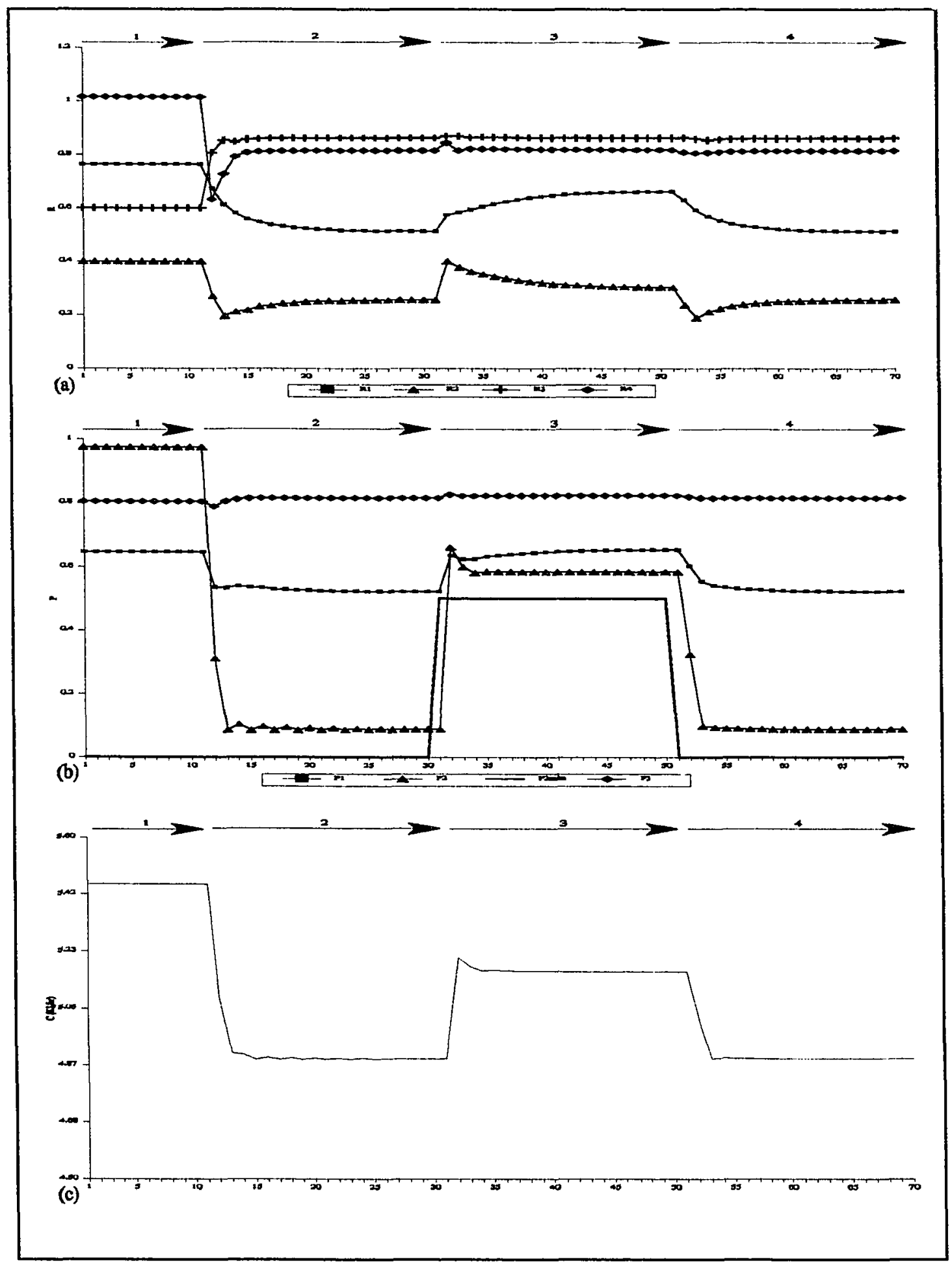

Figure 7-3. Parallel Reactor Feed Results for Minimizing Costs: (a) Normalized Raw Feeds, (b) Normalized Product Streams, (c) Costs 
sustain the $\mathrm{P}_{2}$ production above its minimum, more of the cheap raw feeds is required as less of the expensive raw feeds is used. The cost levels off during step 2 as product $P_{2}$ nears its lower limit and raw feeds $R_{3}$ and $R_{4}$ approach their upper constraints due to the parabolic penalty function used on the constraint errors. During the third run when the minimum of product $P_{2}$ is increased, the cost jumps up primarily due to the increase in the first and second raw feeds $\left(R_{1}, R_{2}\right)$ since they are the most expensive raw materials. The third and fourth raw feeds $\left(R_{3}, R_{4}\right)$ remain essentially at their maximum to try to meet the increased demand. During run four when the minimum for $P_{2}$ is lowered, the cost drops again because $R_{1}$ and $R_{2}$ usage decreases and levels out as the lower constraint of $P_{2}$ begins to take control. What is important about this last set of runs is that several manipulated variables go near their limits and the feed to reactor five bottoms out. This manipulated variable saturation is investigated next.

To test the idea of the extended controller discussed earlier, another set of runs is carried out identical to the first, where profits are maximized, except the maximum feed to reactor one is lowered from 100 to $90 \mathrm{Klb} / \mathrm{hr}$. As a base case, the regular SMCO controller is used and those results are shown in Figure 7-4. The runs are then repeated using the extended controller and that data is also shown in Figure $7-4$ but identified with an asterisk $\left(^{*}\right)$ following the variable names. At first glance there appears to be no difference between the regular and extended cases, but upon closer examination, the cost for the enhanced operation is about $\$ 100 / \mathrm{hr}$ lower than that for the regular one. This slight decrease is due to the higher $P_{1}$ and $P_{2}$ 


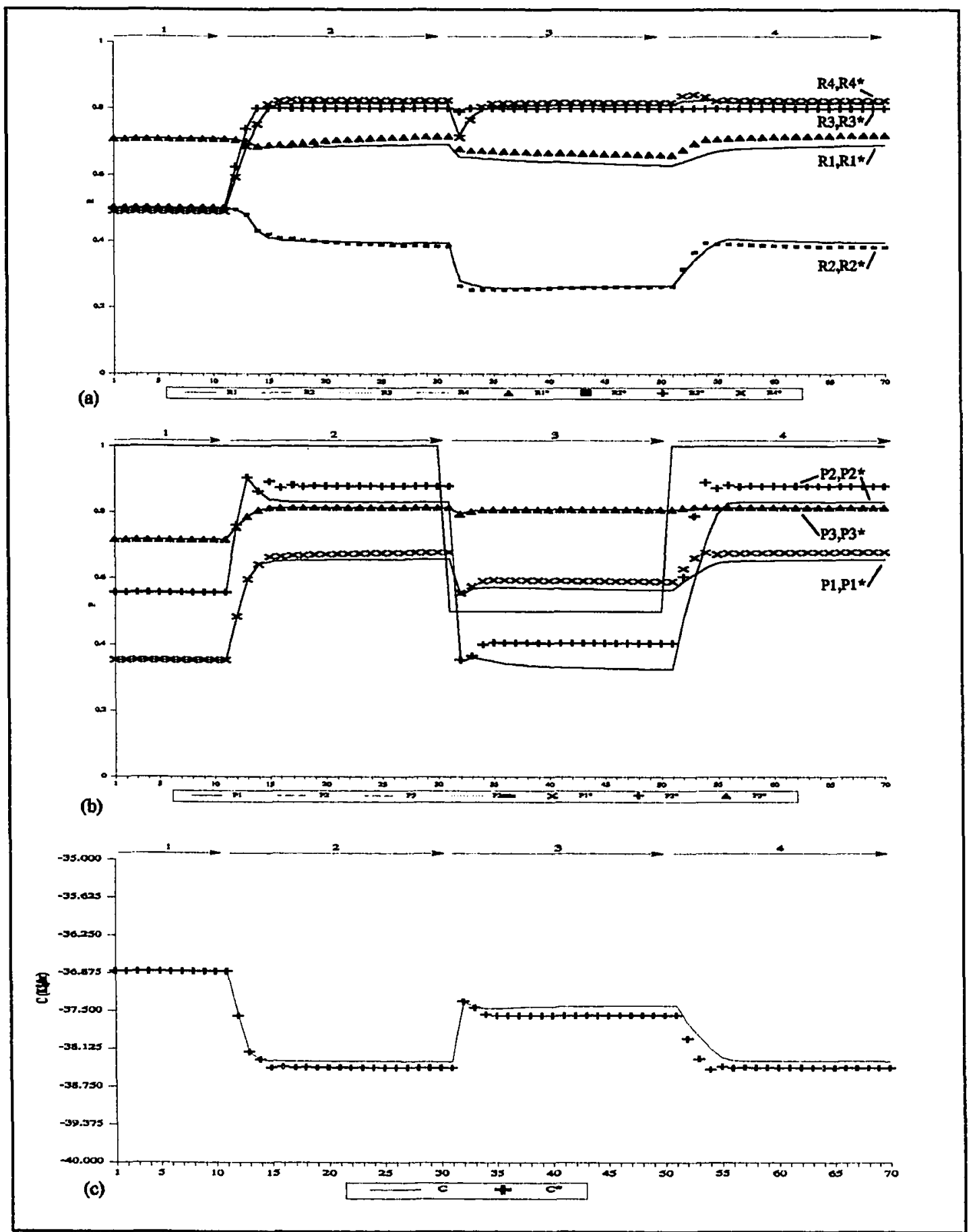

Figure 7-4. Parallel Reactor Feed Results with extended controller: (a) Normalized Raw Feeds, (b) Normalized Product Streams, (c) Costs 
production using the modified controller. The limiting factor in these runs is product $\mathbf{P}_{2}$ approaching its upper limit as in the first set of runs. It turns out that more feed is being pushed through reactor five with the extended controller as opposed to the regular controller to make up for the limited flow to reactor one. Therefore, the extended controller could be economically beneficial when some manipulated variables are saturating.

\section{Summary}

A parallel reactor feed rate application for the Supervisory Multivariable Constrained Optimization has been presented along with its performance in minimizing production costs. The algorithm has been carried out in an industrial distributed control system for a parallel furnace feed rate problem. The idea of an extended controller has been illustrated where saturating manipulated variables are removed from the vector set that the optimization algorithm considers. This technique can reduce costs and can be used to keep the optimization active while certain low-level controllers are off-line. By reducing the process model to a steady-state gain matrix, a compact algorithm can be created which allows for any number of constrained and manipulated variables. The user selects an economically relevant cost function and then provides for the calculation of the gain matrix and cost partials for a particular application. 


\section{Chapter 8}

\section{Conclusions}

Introduction

The preceding chapters have presented an algorithm for Supervisory Multivariable Constrained Optimization (SMCO) and analyzed its performance in optimizing a single distillation column, a train of columns, and the feeds to a set of parallel reactors. In this final chapter, the objectives of the research as proposed in Chapter 1 are reviewed to see what has been achieved. The contributions of the SMCO algorithm to the field of on-line optimization are then discussed. Finally, suggestions for future work are given based on issues that arose during the course of the investigation.

\section{Goals Reached}

One of the primary goals of the algorithm was to make it simple yet effective. Towards this end, assuming the process reaches steady state between optimization moves greatly simplified the model identification as well as the control implementation. Along the same lines, the algorithm was assumed to act in a supervisory capacity directing low-level regulatory controllers which would be responsible for the dynamics of the process. Following these assumptions, an algorithm was developed that was compact enough to implement on a distributed control system with limited resources for an industrial distillation train and a network 
of parallel reactors. Despite its compactness, SMCO incorporates inequality constraints, control move suppression, and cost reduction. The inequality constraints are included as set-point errors but the set points become the midpoints of the constrained ranges. A parabolic penalty function is then used to modify the errors so when the constrained variable is away from its limits, the error term is essentially zero and the cost minimization is driving the control moves. Move suppression is also included in a manner similar to DMC to prevent large incremental changes in the control moves. These move suppression parameters become tuning parameters for the algorithm. The cost minimization is included in the algorithm through cost partials which describe how the cost behaves in response to the dependent (constrained) and independent (manipulated) variables. This cost influence is balanced against constraint violations using a cost weight factor which becomes another tuning parameter for the algorithm.

The structuring of the SMCO platform is flexible enough to handle a single distillation column as well as a whole distillation train. Basically, there are three processing stages for implementing the optimization. The first or pre-processing stage evaluates error terms, cost, and cost partials to be used in the algorithm. In this first phase, on-line gains are also estimated from on-line measurements using energy and mass models of the process. The pre-processor can be constructed so it automatically compensates for the addition or removal of constraints as long as the steady state gain matrix is updated after each change. The second stage performs the optimum control move calculations using the pre-processor information. This 
structuring allows a generic processor to be developed for the second stage that can be used for any application and moved from one platform to another with little modification. The final stage of the strategy evaluates the control moves and passes valid set points to the low-level regulatory controllers at the proper intervals. In this post-processor, saturation of manipulated variables can be detected and dealt with by setting the appropriate flags.

\section{Contributions}

The major contribution to on-line optimization is a compact yet versatile algorithm that minimizes costs while satisfying multiple process constraints. The key to this simplification is the assumption that the process reaches steady state or process variables basically level out with some minor fluctuations between optimization updates. The steady state settling times can vary from several minutes to several hours depending on the process. Using this approach, the optimization acts as a supervisory controller that passes optimum set points to low-level controllers. These regulatory controllers handle the process dynamics in between optimization updates. The steady state assumption also reduces the process model size which reduces the identification phase and makes control implementation easier.

Another contribution is the manner in which inequality constraints are incorporated into the optimization. The normal set point error terms are modified by replacing the set point with the lower or upper constraint or some combination of the two. The errors are then penalized by a function to indicate when the constraint 
should take priority over the cost minimization. In this research, a parabolic penalty function is chosen over a simple step and a cubic function because it provides a wide enough region where the constraint is inactive so cost can be minimized yet enough of a buffer zone so the constraint is not be violated.

The incorporating of the cost in the control move calculation and allowing it to be a non-linear function of the independent and dependent variables is another contribution to on-line optimization. Most on-line techniques either perform the cost minimization in a separate procedure or only allow the cost to be a linear function of the variables. SMCO also breaks the cost partials up into independent and dependent terms using the steady state gains. The influence of the cost in the optimization is adjusted using a cost weight which becomes a tuning parameter for the algorithm.

From the experiments conducted in this research another contribution to online optimization is the development of two methods to minimize the cost of a train of interconnected units, like the distillation train of Chapters 5 and 6 . These two techniques are identified as: 1) Independent which uses a dynamic programming approach, and 2) Total which considers the entire train as one unit. The Independent method requires setting SMCO up for each unit in the train and calculating an interconnecting stream value as the cost of that unit divided by the flow rate of the connecting stream. The units are then processed starting with the last one in the train and proceeding back up the line. The Total technique requires the same set-up as for the Independent method for each unit in the train but then requires the additional compilation of "cross" gains and "cross" cost partials. The cross gains represent how 
the manipulated variables of one column affect another column downstream via the interconnecting stream. In the same manner, the "cross" cost partials indicate how the manipulated variables of one column affect the costs of columns downstream through the interconnecting stream. The appropriateness of each method is discussed next in relation to the tests performed in this research.

Judging from the experiments carried out, if none of the manipulated variables are saturating at their limits, then either method will produce the same amount of savings as shown in Figure 5-5. However, if the manipulated variables of one of the units in the train are at their limits and there is a constraint shift which requires the variables to go beyond these borders, the independent method cannot rectify the situation. In this scenario, the Total technique is preferred since it looks at the whole train and can adjust the manipulated variables of an upstream unit to try to correct the constraint violation. This result is demonstrated in Figure 6-1. As far as columns not reaching steady state by the next optimization sample, no evidence was found that showed one mode preferable to another as discussed in Chapter 6. However, if there are significant differences in the steady state settling times of the columns or units in a train, the independent mode is preferable since individual update intervals can be set up for each instance of SMCO. Considering these findings and the extra set-up of "cross" gains and "cross" cost partials for the Total method, the Independent technique should be used unless saturation of manipulated variables is expected. The Total technique can always be added with minimal effort for a train where the units 
have already been set up for the Independent method. The train can then be optimized using either techniques.

Another contribution to on-line optimization is the way in which SMCO handles its manipulated variables when they become saturated. In any optimization, if some of the independent (manipulated) variables hit their limits before the dependent (constrained) variables reach theirs, a lower cost may be achievable by modifying the moves in the unconstrained variables. To take advantage of this, an extended controller was developed where the saturated manipulated variables are removed from consideration and the algorithm is re-called with a reduced manipulated vector set. In addition to this saturation problem, this logic can be used to take low-level controllers off-line for repair or maintenance while the optimization continues with the remaining controllers. Chapter 7 demonstrated that the extended controller can save as much or more money as the normal controller given the same weights. For these reasons, the extended controller concept should be incorporated into the optimization algorithm. The additional computations for the iteration of the optimum move equation are trivial considering the time between steady states, i.e. hours, and the simple mathematics involved for modern computers.

\section{Future Work}

Future work should be performed on automating the identification of the steady state gains not estimated on-line. Any multivariable regression package or routine could be used for this purpose. The predicted values from the steady state 
process model could be monitored and when the model error exceeded some tolerance, the gains could be re-identified. The other on-line gains would automatically update since they are deduced using the mass and energy models of the system as shown in Chapter 5. Further studies should also be conducted to document that the Independent mode is superior to the Total mode when columns have different steady state settling times. Another field experiment could be conducted using the "extended" form of the algorithm to investigate this and other issues. The field application would also allow an opportunity to further examine the effects of not reaching steady state between optimization updates. The groundwork has already been laid for such an investigation using a total distributed control system such as Honeywell's TDC 3000 system. The algorithm could also be applied to other common industrial processes like catalytic cracking or hydrocracker fractionation.

\section{Summary}

By reducing the process model to a steady-state gain matrix, a compact algorithm can be created as shown in Chapter 3 which allows for multiple constraint monitoring with cost reduction. This Supervisory Multivariable Constrained Optimization algorithm is concise enough to allow implementation on almost any computer platform yet flexible enough to handle a single unit like a distillation column as well as a whole process like a distillation purification train. Two methods, Independent and Total, are provided for minimizing the overall costs for a process train depending on the given conditions. The user needs to decide on an 
economically relevant cost function and then provide for the calculation of the gain matrix and cost partials for a particular application. The performance of the algorithm can then be tuned using the move suppression terms and the cost weight. Results have been presented which show that SMCO can minimize the cost of an ethylene purification train even if some of the columns do not reach steady state between the optimization samples. It has also been demonstrated that SMCO can minimize the cost for a parallel reactor network even if some manipulated variables are saturating. 


\section{Bibliography}

Broyden, C.G., "A New Method for Solving Nonlinear Simultaneous Equations", Computer Journal, 1969, vol. 12, pp. 94-99.

Chemical Engineering, McGraw-Hill Publishing Company, New York, May 1993.

Chemical Marketing_Reporter, Schnell Publishing Company, New York, February $15,1993$.

Chi Yiliang, A Study of the Dynamic Behavior of Distillation Columns, Masters

Thesis, Chemical Engineering, Louisiana State University, December 1992.

Corripio, Armando B., "Z-Transform Block Diagram Analysis," AIChE Modular Instruction Series, Module A3.4, New York: AIChE, 1982.

Cutler, Charles R., and B. L. Ramaker, "DMC - A Computer Control Algorithm," National Meeting of AIChE, April, 1979, Houston, TX.

Cutler, Charles R., and R. T. Perry, "Real Time Optimization with Multivariable Control is Required to Maximize Profits," National Meeting of AIChE, April, 1983, Los Angeles, CA.

Cutler, Charles R., A. M. Morshedi, and J. J. Haydel, "An Industrial Perspective on Advanced Control," National Meeting of AIChE, October, 1983, Washington, D. C.

Cutler, Charles R., and C. R. Johnston, "Analysis of the forms of the Dynamic Matrix," Meeting of the Instrument Society of America, October 21-24, 1985, Philadelphia, Pennsylvania.

Cutler, Charles R., and R. B. Hawkins, "Application of a Large Predictive Multivariable Controller to a Hydrocracker Second Stage Reactor," 1988 American Control Conference, June 15 - 17, Atlanta, GA.

Froisy, J. Brian, and Jacques Richalet, "Industrial Applications IDCOM," Chemical Process Control - III, 1986, Elsevier, New York.

Garcia, Carlos E., and Manfred Morari, "Optimal Operation of Integrated Processing Systems, Part I: Open-Loop On-Line Optimizing Control," AIChE Journal, Nov, 1981, vol. 27, No. 6, p. 960. 
Georgiou, Apostolos, Christos Georgakis, and William L. Luyben, "Nonlinear Dynamic Matrix Control for High Purity Distillation Columns," $\underline{\text { AIChE }}$ Journal, August 1988, vol. 34, No. 8, pp. 1287-1298.

Henley, Ernest J., and J. D. Seader, Equilibrium-Stage Separation Operations in Chemical Engineering, John Wiley and Sons, New York, 1981.

Jang, Shi-Shang, Babu Joseph, and Hiro Mukai, "On-line Optimization of Constrained Multivariable Chemical Processes," AIChE Journal, Jan, 1987, vol. 33 , no. 1 , pp. 26-35.

Kennedy, J. Patrick, "A Simple Steady State Multivariable Control Algorithm with Examples of Gasoline Blending and an Ammonia Reform Furnace," Summer Simulation Conference Proceedings, July 21-23, 1975, San Francisco, CA.

Koninckx, Jan, Thomas J. McAvoy, and Thomas E. Marlin, "On-line Optimization using Steady-State Models," AIChE Annual Meeting, 1988, Washington, D.C.

Marchetti, Jacinto L., Duncan A. Mellichamp, and Dale E. Seborg, "Predictive Control Based on Discrete Convolution Models," Industrial and Engineering Chemistry Process Design and Development, 1983, vol. 22, pp. 488 - 495.

Martin, G. D., "Long-Range Predictive Control," AIChE Journal, vol. 27, no. 5, September, 1981, pp. 748 - 753.

Martin, G. D., L. D. VanHorn, and K. M. Cassaday, "Experience with Hydrotreater Computer Control," Hydrocarbon Processing, vol. 63, No. 3, March, 1984, pp. $66-70$.

McFarlane, Randall C. and David W. Bacon, "Adaptive Optimizing Control of Multivariable Constrained Chemical Processes: 1. Theoretical Development," Industrial Engineering Chemical Research, 1989, vol. 28, pp. 1828-1834.

Moore, Robert D., and Armando B. Corripio, "On-Line Optimization of Distillation Columns in Series," Chemical Engineering Communications, 1991, vol. 106, pp. $71-86$.

Pike, Ralph W., Optimization for Engineering Systems, Van Nostrand Reinhold Company, New York, 1986.

Peters, Max S. and Klaus D. Timmerhaus, Plant Design and Economics for Chemical Engineers, 3rd edition, McGraw-Hill Book Company, New York, 1980. 
Press, William H., Numerical Recipes in Fortran, Cambridge University Press, New York, 1992.

Ramsey, Jr., John R. and Patrick B. Truesdale, "Blend Optimization Integrated into Refinery-wide Strategy," Oil and Gas Journal, March 19, 1990.

Richalet, J., A. Rault, J. L. Testud, and J. Papon, "Model Predictive Heuristic Control: Applications to Industrial Processes," Automatica, 1978, vol. 14, pp. 413 - 429.

Rosendorf, P., M. Kubicek, and J. Schöngut, "On-line Optimization of a Rectification Column," Computers and Chemical Engineering, 1988, vol. 12, no. $2 / 3$, pp. 199-203.

Sourander, M. L., M. Kolari, J. C. Cugini, J. B. Poje, and D. C. White, "Control and Optimization of Olefin-Cracking Heaters," Hydrocarbon Processing, June 1984, pp. 63-69.

Tomlins, Aric H., and Thomas R. Thieme, "Application of Predictive Multivariable Control to a Fluid Catalytic Cracking Process," Proceedings of ISA/1989 International Conference/Exhibition in Advanced Instrumentation, paper \#89-0496.

Tran, David, and Charles Cutler, "Dynamic Matrix Control on Benzene and Toluene Towers," Proceedings of ISA/1989 - International Conference/Exhibition in Advanced Instrumentation, paper \#89-0498. 


\title{
Appendix A
}

\author{
Symbols \\ $\underline{\underline{A}}=$ Process steady state gain matrix \\ $\mathrm{B}=$ Bottoms flow in Klbmole/hr \\ $\underline{B}=$ Reactor feed breakdown or allocation matrix \\ $\mathrm{C}(\mathrm{Y}, \mathrm{U})=$ Cost function in terms of $\mathrm{Y}$ and $\mathrm{U}$ \\ $D_{c}=$ Distillate condensate $\left(D_{c}=D_{1}+R\right)$ in Klbmole/hr \\ $D_{1}=$ Distillate liquid flow in Klbmole/hr \\ $\mathrm{D}_{\mathrm{v}}=$ Distillate vapor flow in Klbmole/hr \\ $\mathrm{DC} / \mathrm{D} \Delta \underline{\mathrm{U}}=$ Cost partial vector \\ $\underline{E}=$ Error vector for constraints \\ $E_{i}=$ Error for constraint $i$ \\ $\mathrm{E}_{\mathrm{i}}^{0}=$ Error when no moves are made \\ $\mathrm{F}=$ Column Feed flow in Klbmole/hr \\ $F_{i}=$ Reactor Feed in $\mathrm{Klb} / \mathrm{hr}$ \\ $F_{v}=$ Maximum vapor boil-up rate in column \\ $\mathrm{H}_{\mathrm{B}}^{\text {vap }}=$ heat of evaporation of bottoms \\ $\mathrm{H}_{\mathrm{b}}=$ Enthalpy of bottom in MBtu/Klbmole \\ $H_{D}^{\text {vap }}=$ heat of condensation of distillate \\ $\mathbf{H}_{\mathrm{dl}}=$ Enthalpy of Distillate Liquid in MBtu/Klbmole \\ $\mathrm{H}_{\mathrm{dv}}=$ Enthalpy of Distillate Vapor in MBtu/Klbmole \\ $\mathrm{H}_{\mathrm{vf}}=$ Enthalpy of the saturated vapor leaving the feed tray \\ $\mathrm{H}_{\mathrm{Lf}}=$ Enthalpy of the liquid leaving the feed tray \\ $\mathrm{H}_{\mathbf{r}}=$ Enthalpy of feed in Mbtu/Klbmole \\ $\mathrm{L}_{\mathrm{s}}=$ Liquid overflow for the stripping section \\ $\mathrm{M}=$ Number of manipulated variables \\ $\mathrm{m}_{\mathrm{b}}, \mathrm{m}_{\mathrm{dl}}, \mathrm{m}_{\mathrm{dv}}=$ Factors for value terms $\mathrm{V}_{\mathrm{b}}, \mathrm{V}_{\mathrm{d} l}, \mathrm{~V}_{\mathrm{d} v}$, respectively \\ $\mathbf{N}=$ Number of constraints \\ $P_{i}=$ Flow rate of the $i^{\text {th }}$ product stream in $\mathrm{Klb} / \mathrm{hr}$ \\ $P_{r}=$ Product recovery fraction \\ $\mathrm{q}=$ thermal condition of the feed \\ $\underline{\underline{Q}}=$ Reactor product yield matrix \\ $\mathbf{Q}_{\mathrm{c}}=$ Condenser energy load in $\mathrm{Mbtu} / \mathrm{hr}$ \\ $\mathrm{Q}_{\mathrm{r}}=$ Reboiler heat load in $\mathrm{Mbtu} / \mathrm{hr}$ \\ $\mathrm{R}=$ Reflux (also liquid overflow for rectifying section) \\ $\mathbf{R}_{\mathrm{i}}=$ Flow rate of the $\mathrm{i}^{\text {th }}$ raw feed stock in $\mathrm{Klb} / \mathrm{hr}$ \\ $\mathrm{U}_{\mathrm{k}}=$ Manipulated variable $\mathrm{k}$ \\ $\mathrm{U}_{\mathrm{k}}^{\max }=$ Upper limit for $\mathrm{U}_{\mathrm{k}}$ \\ $\mathrm{U}_{\mathrm{k}}^{\min }=$ Lower limit for $\mathrm{U}_{\mathrm{k}}$ \\ $V_{b}=$ Value of bottom in $\$ /$ lbmole \\ $\mathrm{V}_{\mathrm{c}}=$ Value of condenser load in $\$ / \mathrm{Kbtu}$
}


$\mathrm{V}_{\mathrm{dl}}=$ Value of liquid distillate in \$/lbmole

$\mathrm{V}_{\mathrm{dv}}=$ Value of vapor distillate in $\$ /$ lbmole

$\mathrm{V}_{\mathrm{pi}}=$ Value of Product $\mathrm{i}$ in $\$ / \mathrm{Klb}$

$V_{t b}=$ Value of reboiler load in $\$ / \mathrm{Kbtu}$

$V_{\mathrm{rk}}=$ Value of Raw Feed $\mathrm{k}$ in $\$ / \mathrm{Klb}$

$\mathrm{V}_{\mathrm{s}}=$ Vapor flow in the stripping section

$\mathrm{V}_{\mathrm{r}}=$ Vapor flow in the rectifying section

$\underline{\underline{W}}=$ Diagonal weight matrix made up of $\mathrm{W}_{\mathrm{i}}$ terms

$\bar{W}_{\mathrm{c}}=$ Weight factor for Cost function

$\mathrm{W}_{\mathrm{i}}=$ Weight of constraint $\mathrm{i}$

$\mathrm{x}_{\mathrm{b} 2}=$ Bottom pseudo-light mole fraction

$\mathrm{x}_{\mathrm{d} 1}=$ Distillate liquid pseudo-heavy mole fraction

$x_{j}=$ Pseudo-light liquid composition of each tray

$y_{\mathrm{d} 1}=$ Distillate vapor pseudo-heavy mole fraction

$y_{i}=$ Pseudo-light vapor composition of each tray

$\mathrm{Y}_{\mathrm{i}}=$ Constrained variable $\mathrm{i}$

$Y_{i}^{0}=$ Previous value of $Y_{i}$

$\hat{\mathrm{y}}_{\mathrm{m}}=$ predicted output at step $\mathrm{m}$

$Y_{i}^{\max }=$ Upper limit for $Y_{i}$

$Y_{i}^{\min }=$ Lower limit for $Y_{i}$

$\mathbf{z}_{1}=$ Feed pseudo-heavy mole fraction

Greek Symbols

$\alpha_{\mathrm{m}}=$ Relative volatility between light and heavy components

$\Delta \mathrm{U}_{\mathrm{j}}=$ Manipulated variable change

$\Delta \mathrm{Y}_{\mathrm{i}}=$ Constrained variable change

$\underline{\underline{\Lambda}}=$ Diagonal matrix of move suppression parameters $\lambda_{\mathrm{j}}$

$\bar{\lambda}_{\mathrm{j}}=$ Move suppression factor for $\Delta \mathrm{U}_{\mathrm{j}}$ 


\title{
Appendix B
}

\section{PC Distillation/SMCO Simulation}

\author{
Introduction
}

The distillation simulation used to generate results for Chapters 5 and 6 is written in Turbo Vision, an object-oriented language from Borland Pascal. Turbo vision provides the benefits of object programming in a window type environment for DOS. The program will run on any 386 or higher IBM compatible machine with $640 \mathrm{~K}$ and a CGA, EGA, or VGA compatible monitor. A mouse is not required but makes operating the program much easier. A disk with the raw code, executable, and sample data files is attached to the back cover. The distillation files on this disk are in a self-extracting executable file called Distill1.exe. To extract the files, copy Distill1.exe to another floppy or hard disk with $950 \mathrm{~K}$ free space and simply run the program which will generate the following files:

\begin{tabular}{|c|c|}
\hline Distill.exe & Steady State Distillation Simulation with SMCO algorithm \\
\hline Distill.pas & Source code for Main Program of Distill.exe \\
\hline Train.pas & Source code for Train Unit used by Distill.pas \\
\hline Distcost.pas & Source code for Distillation Cost Unit used by Train.pas \\
\hline Distmodl.pas & Source code for Distillation Model Unit used by Distcost.pas \\
\hline DistCols.hlp & Help file for Distillation Columns \\
\hline DistCost.hlp & Help file for Distillation Costs \\
\hline DistCtrl.hlp & Help file for Distillation Controls \\
\hline DistGen.hlp & Help file for Distillation General Information \\
\hline DistIndx.hlp & Help file for Distillation Indexing System \\
\hline DistMisc.hlp & Help file for Distillation Miscellaneous Operations \\
\hline DistModl.hlp & Help file for Distillation Models \\
\hline DistRun.hlp & Help file for Distillation Runs \\
\hline Demeth.col & Demethanizer Column file used in Chapter 5 \\
\hline Train1I.trn & $\begin{array}{l}\text { Ethylene Purification Train file used in Independent runs in } \\
\text { Chapters } 5 \text { and } 6 \text { with composition set points as manipulated } \\
\text { variables }\end{array}$ \\
\hline Train1T.tm & $\begin{array}{l}\text { Ethylene Purification Train file used in Total runs in Chapters } 5 \\
\text { and } 6 \text { with composition set points as manipulated variables }\end{array}$ \\
\hline Train2I.trn & $\begin{array}{l}\text { Ethylene Purification Train file used in Independent runs in } \\
\text { Chapters } 5 \text { and } 6 \text { with manipulated feed }\end{array}$ \\
\hline Train2T.trn & $\begin{array}{l}\text { Ethylene Purification Train file used in Total runs in Chapters } 5 \\
\text { and } 6 \text { with manipulated feed }\end{array}$ \\
\hline
\end{tabular}

Once the files have been extracted, the self-extracting executable can be deleted. 
Also contained on the disk is a self-extracting executable called Common.exe which contains common files used by the distillation and reactor simulations Extract these files to the same location as the distillation files noting that about $310 \mathrm{~K}$ of free space will be needed initially to hold the self-extracting executable and the extracted files. Again the self-extracting executable can be deleted once the files have been extracted. The files in Common.exe are:

SSModel.pas - Source code for Steady State Model Unit

Output.pas - Source code for Output Unit used by SSModel.pas

Invert.pas - Source code for Invert Unit used by SSModel.pas

Vesa16.bgi - Graphics driver for Vesal6 screens

PC3270.bgi - Graphics driver for PC3270 screens

IBM8514.bgi - Graphics driver for IBM8514 screens

Herc.bgi - Graphics driver for Hercules screens

EGAVGA.bgi - Graphics driver for EGA or VGA screens

CGA.bgi - Graphics driver for CGA screens

ATT.bgi - Graphics driver for ATT screens

Litt.chr - Little font used by Graphic routines

Outputs.hlp - Help file for Outputs

Graphs.hlp - Help file for Graphs

Once the distillation and common files have been extracted to the same location, the Distillation/SMCO simulation can be run by entering DISTILL at the DOS prompt. The only files absolutely necessary to run the distillation program are distill.exe, litt.chr, and the *.bgi files. The *.hlp files are only used if one tries to access Help on-line.

The following sections describe how the program is set up and what various options are available to the user. Note that some variable names are written to match those shown in the program. For example, Xd really represents $x_{d}$ or Ns really represents $\mathbf{N}_{\mathrm{g}}$. The same information presented below is available on-line via the Help option on the Menu Bar.

\section{General Information}

Distill.exe is a testing tool for the Supervisory Multivariable Constrained Optimization algorithm or SMCO for short. The goal of SMCO is to minimize costs while meeting design constraints for multivariable systems that come to steady state between samples. SMCO acts in a supervisory capacity directing lower level controllers (LLC).

The program has a steady state binary distillation column simulation built in to test the performance of SMCO. Each column has a feed $(F)$, distillate vapor (Dv), 
distillate liquid (Dl), and bottoms (B) stream. A partial reboiler and condenser are used where the heat load to the reboiler $(\mathrm{Qr})$ and the reflux rate $(\mathrm{R})$ can be specified. Constant molar overflow, column pressure, and relative volatility are assumed in each column.

A single or multiple column simulation is possible with SMCO acting in "INDEPENDENT" or "TOTAL" control modes. In "INDEPENDENT" control, SMCO uses a dynamic programming approach to minimize total costs. For distillation trains in this mode, the last column is optimized first and then the value of the intermediate or connecting stream (Vi_now) is calculated as the column's cost per unit feed $(\mathrm{C} / \mathrm{F})$ and passed to upstream columns. This mode is preferable if each column has a significantly different settling time. In "TOTAL" control, SMCO treats all columns as one unit when reducing the total operating costs. In this mode, the values of the intermediate streams are set to zero.

When the program is started, a default column exists in the train which simulates a Demethanizer column. You may also load another column from a disk by choosing the Load option under SS Columns. The program searches the current directory for files ending with *.col. You may also load in a Train of Columns by choosing Train then Load under SS Columns- Train.

When building a column, the product stream needs to be identified for costing purposes. For a train of columns, the stream which feeds subsequent columns also needs to be identified. Use Settings under SS Columns to make these choices.

The cost function for the distillation simulation is written as a sum of the material and energy streams weighted by their economic values:

Cost $=\mathrm{Qr} \mathrm{Vr}+\mathrm{Qc} \mathrm{Vc}+\mathrm{B} \mathrm{Vb} \mathrm{mb}+\mathrm{Dl} \mathrm{Vdl} \mathrm{mdl}+\mathrm{Dv} \mathrm{Vdv} \mathrm{mdv}$

where Vdl, Vdv, $\mathrm{Vb}$ are values in \$/Klbmole; Vr, Vc are values in \$/MBtu; B, Dl, $\mathrm{Dv}, \mathrm{F}$ are flows in $\mathrm{Klbmole} / \mathrm{hr}$; Qr, Qc are heat loads in $\mathrm{MBtu} / \mathrm{hr}$; and $\mathrm{mb}$, mdl, mdv are factors for default material stream values. All material and energy streams carry a positive sign while their values (Vi) reflect either a profit, if negative, or a debit, if positive. The cost function is discussed in more detail in the Cost section below.

If NO Low Level Controller (LLC) is desired then resize the LLC Index vector (IndexYl) to 0 . If NO DISTURBANCES are desired then resize DistPct to 0 under Controller - Weights. Note that each matrix or vector can be edited on screen and its properties such as name and size can be modified by pressing the Props button.

Note that the user may call up help screens under the Help option. 
To clear any window just click its square in the upper left hand corner. The term "click" means to position the mouse pointer over the object of interest and press the left mouse button once. If a mouse is unavailable, use the Windows menu option close (Alt-F3). Windows can be resized by dragging the lower right corner with the mouse or by choosing Window-Size/Move (Ctrl-F5) and pressing the shift and arrow keys at the same time. To return back to the desktop press the ENTER key. To drag with a mouse, click the left mouse button and hold it as the mouse is moved. To move a window just drag its title bar or choose Windows-Size/Move (Ctrl-F5) and use the arrow keys. Again press ENTER after finished moving window with the keyboard. To enlarge a window to full screen click the arrow in the upper right corner or choose Window-Zoom (F5). To shrink the window back to its original size just click the arrow again or choose Window-Zoom (F5) again. The Window menu offers other options like close-all, cascade, and tile. The Next (F6) and Previous (Shift-F6) Window options jump from one window to the next.

For the dialog boxes (windows with buttons and input boxes), press OK to accept the current data displayed or Cancel to leave without the changes being recorded. Pressing the Escape key is like pressing the Cancel button. The dialog boxes will not go away until either OK or Cancel is pressed. The default button is highlighted and if the return key is pressed, it is like clicking the default button. You may use the TAB key to jump from one input box to another. Note you can shift-TAB to move backwards in the input blocks. You may also tab from one button to another.

To exit the program, press Alt-Q or click it in the lower left hand corner of the status line at the bottom.

\section{SS Columns Option}

The SS Columns menu item allows the user to work with Steady State (SS) Columns in the Train and the Train itself.

Under Settings, the Column Name can be changed. Note that this name may be up to 256 characters long but ONLY the first 8 characters are used as the file name for column storage. When a column is saved, all of its parts including graphs are also saved. An extension of *.col is automatically added to the filename. The Output File specifies where output data is directed for each run. The data is stored as ASCII or text in a comma separated variable format. The file has an extension *.csv and can be read into almost any spreadsheet. The Output Type specifies whether output data is presented in a molar or mass basis. The Preset Flow option tells the program which flow to keep at its preset value. The SMCO Mode indicates the status of the controller for the Independent Control runs, not the Total Control runs. In Auto, SMCO will minimize cost while trying to meet the specified 
constraints when the simulation is Run under Indep Control option. When the simulation is run in Indep Control and SMCO is in manual, step testing and SS Gain Matrix data collecting can be performed. See Run Section for more information. Use the Extended option to activate the extended controller during Independent Runs. HOWEVER, USE WITH CAUTION AS THE EXTENDED OPTION WAS NOT FULLY TESTED WITH THE DISTILLATION SIMULATION MODELS. The Error Penalty option indicates the function used to penalize constraint errors. The Column Product can be specified as the Liquid Distillate, the Vapor Distillate, or the Bottoms. The Feed to the next column in the train can also be specified as one of these streams.

Under Properties, various column properties can be viewed and edited. The product spec is a mole fraction cut-off point where the value of the product switches sign. If have bottoms product then switches sign if pseudo-light concentration goes above this value. If have liquid or vapor distillate product then switches sign if pseudo-heavy concentration goes above this value. If this effect is undesired then set product spec to 0.9999 .

The list of parameters under Properties contains constants and the initial material and energy stream values. The definition of these parameters are given below. Note that [1] refers to the Pseudo-Heavy Component while [2] stands for the Pseudo-Light Component.

$$
\begin{aligned}
& \text { Variable }=\text { Description } \\
& \text { Ns = Stripping Stages includes Partial Reboiler } \\
& \mathrm{Nr}=\text { Rectifying Stages includes Feed Stage and Partial Condenser } \\
& \text { AlphaM = Average Relative Volatility } \\
& \mathrm{Q}=\text { Feed Thermal Condition (see below) } \\
& \mathrm{Wc}=\text { Cost Weighting } \\
& \text { ZeroEps }=\text { Zero Tolerance for convergence } \\
& \text { FlagError }=\text { Min Error to activate Warning Boxes } \\
& \text { Exp Filter }=\text { Exponential filter for variables used by } \operatorname{SMCO}\left(Q_{\text {fil }}\right) \\
& \mathrm{Z2}=\text { Feed Mole Fraction of Pseudo-Light compound } \\
& \text { YD1 = Dist. Vap. Mole Fraction of Pseudo-Heavy compound } \\
& \mathrm{XD1}=\text { Dist. Liq. Mole Fraction of Pseudo-Heavy compound } \\
& \mathrm{XB} 2=\text { Bottom Mole Fraction of Pseudo-Light compound } \\
& \text { MW1 }=\text { Molecular Weight of Pseudo-Heavy Compound ( } \mathrm{lb} / \mathrm{lbmole}) \\
& \text { MW2 = Molecular Weight of Pseudo-Light Compound (lb/lbmole) } \\
& \mathrm{F}=\text { Feed flow } \quad(\mathrm{Klbmole} / \mathrm{hr}) \\
& \text { D1 = Distillate Liquid Flow (Klbmole/hr) } \\
& \text { Dv = Distillate Vapor Flow (Klbmole/hr) } \\
& \mathrm{R}=\text { Reflux Flow (Klbmole/hr) } \\
& \mathrm{B}=\text { Bottoms Flow (Klbmole/hr) }
\end{aligned}
$$




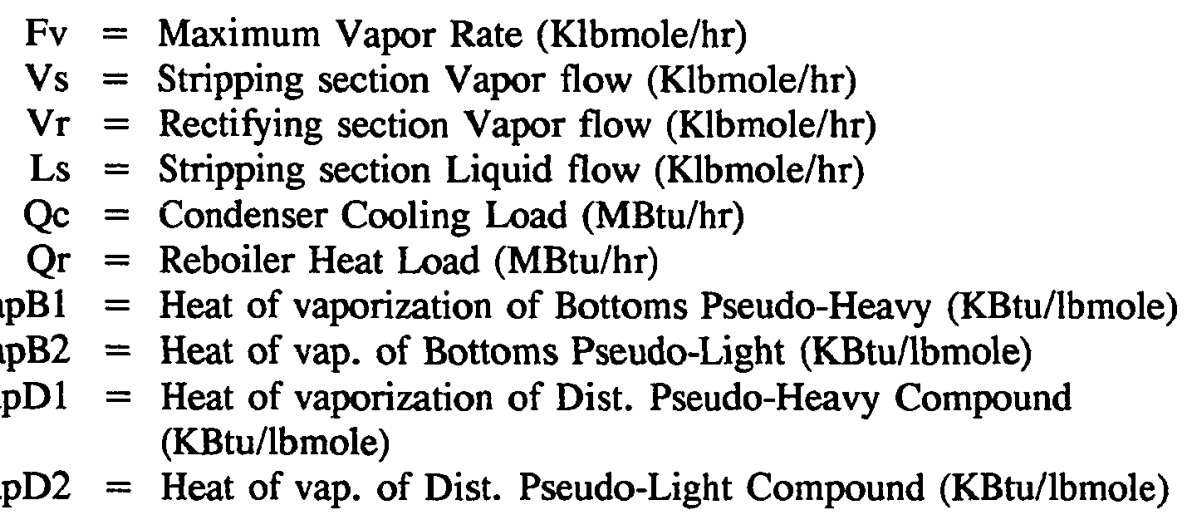

Note:

$Q<0:$ Superheated Vapor

$\mathrm{Q}=0$ : Saturated Vapor

$0<Q<1:$ Liquid and Vapor

$\mathbf{Q}=1:$ Saturated Liquid

$Q>1:$ Subcooled Liquid

The exponential filtering is used to simulate a first order lag. The lagged (filtered) values are calculated by:

$$
Y^{\operatorname{lag}}:=Y^{\operatorname{lag}_{i-1}}+\left(1-Q_{f i}\right)^{*}\left(Y_{i}-Y^{\operatorname{lag}}{ }_{i-1}\right)
$$

where subscripts $i$ and $i-1$ represent the current and previous values of the variables.

The export and import buttons allow data transfer to a disk of information on screen.

The Output item under SS Columns will bring up a window which displays current Column Output. The windows may be used to monitor a column's output during a simulation run. Note, the values displayed in the Output Window are the same ones written to the Output File specified under Settings.

The Run option runs the simulation in either Independent or Total Control. More information about running the program is in the Run section.

The Load/Save/Delete option loads/saves/deletes a column in the train. When any of these options are chosen, a list of columns in the train is displayed. The user then highlights which column to delete or save. Note that a column will be added after the position highlighted. THE PROGRAM WILL ONLY LOAD AS MANY COLUMNS AS MEMORY ALLOWS. Once the maximum is reached, the Load option only displays the current list. When a column is saved, all of its parts 
including graphs and outputs are also saved. An extension of *.col is used for a column file.

The Train Item allows editing, loading, and saving of the current train which is made up of all columns currently in memory. Only one train at a time can be in memory and the first 8 letters of its name are displayed on the status line. Due to DOS limitations the first 8 characters of the train name are used for the train file which has an extension of *.trn. A train file can be considered a collection of column files. Note to run the extended controller on the whole train, the option must be turned on under Train-Settings. HOWEVER, USE WITH CAUTION AS THE EXTENDED OPTION WAS NOT FULLY TESTED WITH THE DISTILLATION SIMULATION MODELS.

\section{Controllers Options}

The Controllers menu item gives the user access to each column's SMCO and LLC variables which are grouped as:

(1) Indices

(2) Constraints

(3) Weights

(4) Matrices

(5) Values

(6) Cost Data

Indices consist of:

Index $Y=$ index for SMCO $Y$ constrained variables

IndexU $=$ index for SMCO U manipulated variables

IndexYl $=$ index for Low Level Control $\mathrm{Yl}$ variables

IndexDist $=$ index for Disturbance variables

Uon $=$ integer vector for active (1) or inactive (2) manipulated variables

The absolute index system used in the program is discussed in the Index Section below.

Constraints consist of:

$\mathrm{Ymin}=$ Minimum constraints for SMCO $\mathrm{Y}$ variables

$Y \max =$ Maximum constraints for SMCO $Y$ variables

Ylset $=$ Low Level Controller Set Points

$\mathrm{Umin}=\mathrm{SMCO}$ minimum manipulated $\mathrm{U}$ values

$\mathrm{Umax}=\mathrm{SMCO}$ maximum manipulated $\mathrm{U}$ values

Weights consist of: 


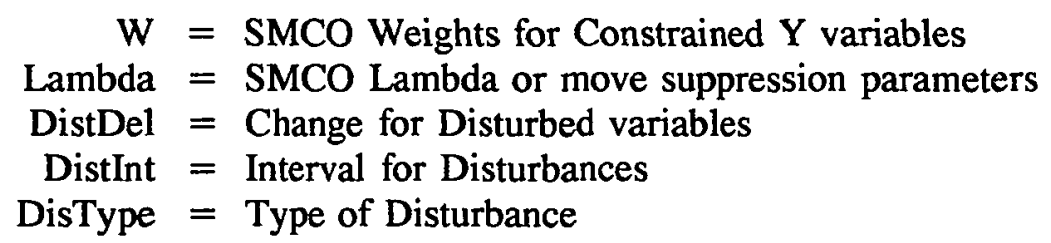

The disturbances are typed as:

1: + DistDel or add DistDel to disturbed variable each DistInt

2: +,- DistDel or alternate adding/subtracting DistDel each DistInt

3: * DistDel or multiply DistDel times disturbed variable each DistInt

4: *,/ DistDel or alternate multiplying/dividing DistDel each DistInt

Matrices consist of:

$\mathrm{A}=$ SMCO Steady State Gains of Column

Limits $=$ lower, upper limits used by Broyden in column model

hMatrix = approximation of inverse Jacobian used by Broyden in column model

Values consist of:

Ypred $=$ SMCO predicted $\mathrm{Y}$ values

States = States of column model

Ftol $=$ function zero tolerances for Broyden method in column model

$Y_{-}$lag $=$lagged (filtered) $\mathrm{Y}$ variables

Cost Data consist of:

DCDU $=$ Derivatives of Cost Fnc. wrt manipulated variables U

DYDF $=$ Derivatives of various variables wrt Feed Flow $F$

DFDU $=$ Derivatives of Feed Flow wrt manipulated variables U

DBDU $=$ Derivatives of Bottoms Flow wrt manipulated variables $U$

DDIDU $=$ Derivatives of Distillate Liquid Flow wrt manipulated variables U

DDvDU $=$ Derivatives of Distillate Vapor Flow wrt manipulated variables $U$

DQcDU $=$ Derivatives of Condenser Cooling wrt manipulated variables $U$

Value $=$ Default Cost values

$\mathrm{H}=$ Stream Enthalpies used in Cost Function

DCX $=$ Misc. Derivatives used in Total SMCO run mode

MValType $=$ Type of multiplier for default cost values

The DCDU, DFDU, and DCX vectors are calculated. The user only has to enter DQcDU, DYDF[1]=Dxd1/DF, DYDF[2]=Dyd1/DF, DYDF[3] $=$ Dxb2/DF, and DYDF[9] $=D Q c / D F$ and ALL other partials will be calculated. If constrained $Y$ includes $F v$ then no need to enter $\mathrm{DQ} c \mathrm{DU}$ since program will calculate. If $\mathrm{Y}$ includes $F v$ and $U$ includes $F$ then no need to enter DQc/DF since program will derive. If $\mathrm{xd} 1, \mathrm{yd} 1$, or $\mathrm{xb} 2$ being controlled by Low Level Controllers (LLC) or are not present in the column, then set their DYDF terms to zero. 
The miscellaneous feed partial vector DYDF is defined as:

$$
\begin{aligned}
& \text { DYDF[1] }=\text { Dxd1/DF }=\text { Change in Liq. Dist. Pseudo-Heavy mole fraction wrt } \\
& \mathrm{F} \\
& \text { DYDF[2] }=\text { Dyd1/DF }=\text { Change in Vap. Dist. Pseudo-Heavy mole fraction wrt } \\
& \mathrm{F} \\
& \text { DYDF[3] }=\mathrm{Dxb} 2 / \mathrm{DF}=\text { Change in Bottom Pseudo-Light mole fraction wrt } \mathrm{F} \\
& \text { DYDF[4] }=\text { DDl/DF }=\text { Change in Distillate Liquid Flow wrt } F \\
& \mathrm{DYDF}[5]=\mathrm{DDv} / \mathrm{DF}=\text { Change in Distillate Vapor Flow wrt } \mathrm{F} \\
& \mathrm{DYDF}[6]=\mathrm{DR} / \mathrm{DF}=\text { Change in Reflux Flow wrt } \mathrm{F} \\
& \mathrm{DYDF}[7]=\mathrm{DB} / \mathrm{DF}=\text { Change in Bottoms Flow wrt } \mathrm{F} \\
& \mathrm{DYDF}[8]=\mathrm{DFv} / \mathrm{DF}=\text { Change in Boil-up Rate wrt } \mathrm{F} \\
& \mathrm{DYDF}[9]=\mathrm{DQc} / \mathrm{DF}=\text { Change in Condenser Cooling wrt } \mathrm{F} \\
& \mathrm{DYDF}[10]=\mathrm{DQr} / \mathrm{DF}=\text { Change in Reboiler Heat wrt } \mathrm{F}
\end{aligned}
$$

The user must enter cost values 1-6:

$$
\begin{aligned}
& \text { Value[1] }=\quad \text { Vf }=\text { Default value of feed in } \$ / \mathrm{bmole} \\
& \text { Value[2] }=\quad \mathrm{Vdl}=\text { Default value of liq. distillate in } \$ / \mathrm{lbmole} \\
& \text { Value[3] }=V d v=\text { Default value of vap. distillate in } \$ / \text { lbmole } \\
& \text { Value[4] }=\quad \mathrm{Vb}=\text { Default Value of bottoms in } \$ / \mathrm{lbmole} \\
& \text { Value[5] }=\quad V c=\text { Default Value of condenser load in } \$ / \mathrm{KBtu} \\
& \text { Value[6] }=\quad \mathrm{Vr}=\text { Default Value of reboiler load in } \$ / \mathrm{KBtu} \\
& \text { Value[7] }=\quad \mathrm{Vi}=\text { Current Intermed Stream value in } \$ / \mathrm{lbmole} \\
& \text { (Value[7] is calculated) }
\end{aligned}
$$

User must enter enthalpy values 1-8:

$$
\begin{aligned}
\mathrm{H}[1]=\mathrm{Hf} 1=\begin{array}{l}
\text { Feed Enthalpy of Pseudo-Heavy Compound } \\
\text { (KBtu/lbmole) }
\end{array} \\
\mathrm{H}[2]=\mathrm{Hf} 2=\begin{array}{l}
\text { Feed Enthalpy of Pseudo-Light Compound } \\
\text { (KBtu/lbmole) }
\end{array} \\
\mathrm{H}[3]=\mathrm{Hd} 11=\begin{array}{l}
\text { Distillate Liquid Enthalpy of Pseudo-Heavy Compound } \\
\text { (KBtu/lbmole) }
\end{array} \\
\mathrm{H}[4]=\mathrm{Hd} 12=\begin{array}{l}
\text { Distillate Liquid Enthalpy of Pseudo-Light Compound } \\
\text { (KBtu/lbmole) }
\end{array} \\
\mathrm{H}[5]=\mathrm{Hdv1}=\begin{array}{l}
\text { Distillate Vapor Enthalpy of Pseudo-Heavy Compound } \\
\text { (KBtu/lbmole) }
\end{array} \\
\mathrm{H}[6]=\mathrm{Hdv2}=\begin{array}{l}
\text { Distillate Vapor Enthalpy of Pseudo-Light Compound } \\
\text { (KBtu/lbmole) }
\end{array} \\
\mathrm{H}[7]=\mathrm{Hb} 1=\begin{array}{l}
\text { Bottom Enthalpy of Pseudo-Heavy Compound } \\
\text { (KBtu/lbmole) }
\end{array} \\
\mathrm{H}[8]=\quad \mathrm{Hb} 2=\begin{array}{l}
\text { Bottom Enthalpy of Pseudo-Light Compound } \\
\text { (KBtu/lbmole) }
\end{array}
\end{aligned}
$$

These enthalpies are used in the cost evaluation. 
The MValType vector indicates the type of multiplier for each default material stream value. Its four elements are $\mathrm{Mf}, \mathrm{Mdl}, \mathrm{Mdv}$, and $\mathrm{Mb}$ for $\mathrm{F}, \mathrm{Dl}, \mathrm{Dv}$, and $\mathrm{B}$, respectively, where:

Type Fnc(mole fraction)

$0 \quad 1.0$ or default

$1 \quad \mathrm{x}_{1}$ or Pseudo-Heavy Compound mole fraction of stream

$2 \mathrm{x}_{2}$ or Pseudo-Light Compound mole fraction of stream

These terms are multiplied times the default material stream values to emphasize the purity's effect on a stream's value. For example, mdv*Vdv is used for the value of the distillate vapor stream. For more information on the column's cost, see Cost Section below.

\section{Index Definition}

The index used for assigning control, manipulated and disturbance variables is listed below:

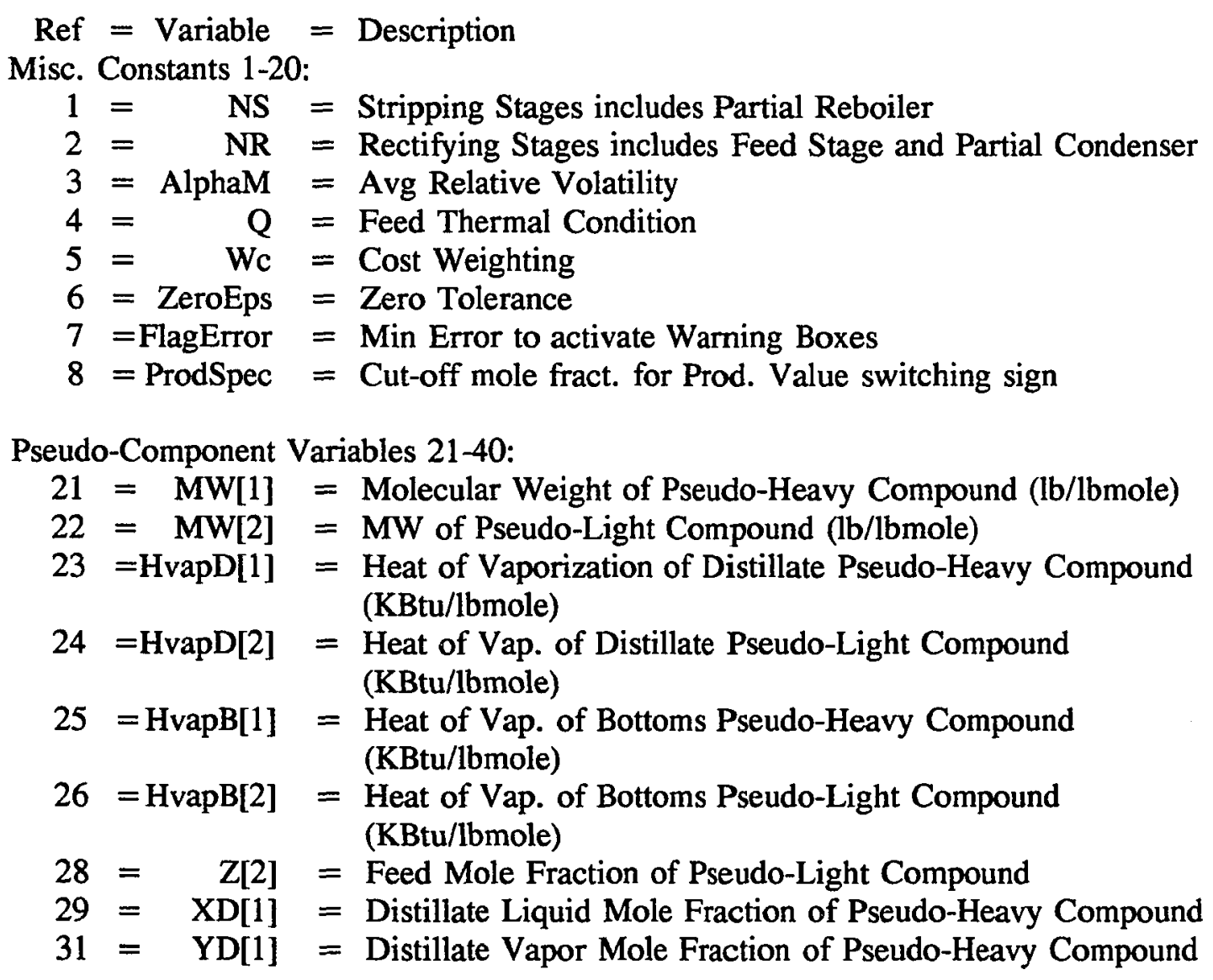


$34=\mathrm{XB}[2]=$ Bottom Mole Fraction of Pseudo-Light Compound

Material and Energy Streams 41-60:

$\begin{array}{rlrl}41 & = & \mathrm{F} & =\text { Feed flow (Klbmole/hr) } \\ 42 & = & \mathrm{Dl} & =\text { Distillate Liquid Flow (Klbmole } / \mathrm{hr}) \\ 43 & = & \mathrm{Dv} & =\text { Distillate Vapor Flow (Klbmole } / \mathrm{hr}) \\ 44= & \mathrm{R} & =\text { Reflux Flow (Klbmole } / \mathrm{hr}) \\ 45= & \mathrm{B} & =\text { Bottoms Flow (Klbmole } / \mathrm{hr}) \\ 46= & \mathrm{Fv} & =\text { Maximum Vapor Rate }(\mathrm{Klbmole} / \mathrm{hr}) \\ 47 & = & \mathrm{Qc} & =\text { Condenser Heat Load }(\mathrm{MBtu} / \mathrm{hr}) \\ 48 & = & \mathrm{Qr} & =\text { Reboiler Heat Load }(\mathrm{MBtu} / \mathrm{hr})\end{array}$

Cost Related Terms 61-100:
$61=$ Cost $=$ Current Cost $(\mathrm{K} \$ / \mathrm{hr})$
$62=$ Value[1] = Default Feed Value $(\$ / \mathrm{lbmole})$
$63=$ Value[2] $=$ Default Distillate Liq. Value $(\$ /$ lbmole $)$
$64=$ Value[3] $=$ Default Distillate Vap. Value $(\$ /$ lbmole)
$65=$ Value[4] = Default Bottoms Value (\$/lbmole)
$66=$ Value $[5]=$ Condenser Heat Value $(\$ / \mathrm{KBtu})$
$67=$ Value[6] = Reboiler Heat Value $(\$ / \mathrm{KBtu})$
$68=$ Value $[7]=$ Intermed. Stream Value $(\$ / 1$ bmole $)$
$69=\mathrm{H}[1]=\mathrm{Hfl}$, Feed Enthalpy of Pseudo-Heavy Compound (KBtu/lbmole)
$70=\mathrm{H}[2]=\mathrm{Hf} 2$, Feed Enthalpy of Pseudo-Light Compound (KBtu/lbmole)
$71=\mathrm{H}[3]=$ Hd11, Dist. Liq. Enthalpy of Pseudo-Heavy Compound (KBtu/lbmole)
$72=\mathrm{H}[4]=\mathrm{Hd} 12$, Dist. Liq. Enthalpy of Pseudo-Light Compound (KBtu/lbmole)
$73=\mathrm{H}[5]=\mathrm{Hdv} 1$, Dist. Vapor Enthalpy of Pseudo-Heavy Compound (KBtu/lbmole)
$74=\mathrm{H}[6]=\mathrm{Hdv} 2$, Dist. Vapor Enthalpy of Pseudo-Light Compound (KBtu/lbmole)
$75=\mathrm{H}[7]=\mathrm{Hb} 1$, Bottom Enthalpy of Pseudo-Heavy Compound (KBtu/lbmole)
$76=\mathrm{H}[8]=\mathrm{Hb} 2$, Bottom Enthalpy of Pseudo-Light Compound (KBtu/lbmole)

LLC Set Points 101-200:

$101=$ Ylset $[1]=$ First LLC set point

$102=$ Ylset[2] = Second LLC set point

Note:

$\mathrm{Q}<0 \quad$ : $\quad$ Superheated Vapor 


$$
\begin{array}{lll}
\mathrm{Q}=0 & : & \text { Saturated Vapor } \\
0<\mathrm{Q}<1 & : & \text { Liquid and Vapor } \\
\mathrm{Q}=1 & : & \text { Saturated Liquid } \\
\mathrm{Q}>1 & : & \text { Subcooled Liquid }
\end{array}
$$

These absolute references are used when defining control, disturbance, and manipulated variables for SMCO and the Low Level Controllers (LLC). For example, if U[1] = LLC Xb2set $=$ Ylset[1] then IndexU[1] $=101$ and Index $Y 1[1]=34$. If $a+/-5$ alternating change in the feed $F$ is desired every 10 steps then DistIndex[1] $=41$, DistDel[1] $=5$, DistInt $[1]=10$, and DisType[1] $=2$. For no disturbances resize or set Dist vector to 0. NOTE disturbances can be applied to ALL columns. Disturbing intermediate streams may lead to erroneous results.

\section{Cost Definition}

The cost function for the distillation simulation is written as a sum of the material and energy streams weighted by their economic values:

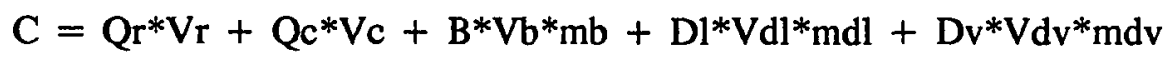

All material and energy streams carry a positive sign while their values (Vi) reflect either a profit, if negative, or a debit, if positive.

The cost function is rewritten in terms of the feed $(\mathrm{F})$, product recovery $(\mathrm{Pr})$, cooling rate $(\mathrm{Qc})$, and either the Distillate Liquid (Dl) or Distillate Vapor (Dv) like:

$\mathrm{C}=\mathrm{F}^{*}\left(\mathrm{a} 1{ }^{*} \operatorname{Pr}+\mathrm{a} 2\right)+\mathrm{a} 3^{*} \mathrm{DI}+\mathrm{a} 4^{*} \mathrm{Qc}$

where:

$$
\begin{aligned}
& \mathrm{a} 1=\mathrm{Vb} * \mathrm{mb}-\mathrm{Vdv}^{*} \mathrm{mdv}+\mathrm{Vr}^{*}(\mathrm{Hb}-\mathrm{Hdv}) \\
& \mathrm{a} 2=\mathrm{Vdv}^{*} \mathrm{mdv}+\mathrm{Vr}^{*}(\mathrm{Hdv}-\mathrm{Hf}) \\
& \mathrm{a} 3=\mathrm{Vdl}{ }^{*} \mathrm{mdl}-\mathrm{Vdv} \mathrm{mdv}^{*}-\mathrm{Vr}^{*}(\mathrm{Hdl}-\mathrm{Hdv}) \\
& \mathrm{a} 4=\mathrm{Vc}+\mathrm{Vr}
\end{aligned}
$$

This form of the column cost is used in evaluating the cost partials DCDU. For example:

DCDU[j] $=F^{*} a{ }^{*}$ DPrDU[j] $+F^{*} P^{*}$ Da1DU[j] + a $2 * D F D U[j]+F^{*}$ Da2DU[j] + a3*DlDU[j] + Dl*Da3DU[j] + a4*DQcDU[j] + Qc*Da4DU[j]

The program will evaluate all these terms based on the column product and the preset flow chosen if the user provides DQcDU, Dxd1/DF, Dyd1/DF, Dxb2/DF, and $D Q c / D F$. If constrained $Y$ includes $F v$ or maximum vapor then no need to enter $D Q c D U$. If $Y$ includes $F v$ and $U$ includes $F$ then no need to enter DQc/DF. If $x d 1$, 
yd1, or $\mathrm{xb} 2$ being controlled by Low Level Controllers (LLC) or are not present in the column, then set their DYDF terms to zero. These partials are evaluated by step testing the column as discussed in the Model Section.

If Exponential filter $\left(Q_{f i l}\right)$ is not zero, then all appropriate terms used in the evaluation of the cost, the cost partials, and the on-line gains will be lagged.

The program uses a model equation like

$\mathrm{Qc}=$ HvapD*Dc$=H v a p D *(V s-D v+(1-q) * F)=H v a p D *(F v-D v+(1-q) * F)=$ HvapD*(Fv+Dl+F*(Pr-q))

to get $\mathrm{DQcDU}=\mathrm{HvapD}\left(\mathrm{DFvDU}+\mathrm{DDIDU}+\mathrm{DFDU} *(\operatorname{Pr}-\mathrm{q})+\mathrm{F}^{*} \mathrm{DPrDU}\right)$.

The model is discussed in the Model Section. Often these expressions simplify. For example, if $\mathrm{Dl}=0$ and $\mathrm{U}<>\mathrm{F}$ then $\mathrm{DQcDU}=\mathrm{HvapD}$ (DFvDU $\left.+\mathrm{F}^{*} \mathrm{DPrDU}\right)$. The DFvDU term comes from the gain matrix and DPrDU is calculated as shown below.

The transformation of the original cost is achieved using over-all mass, energy, and component balances like:

$\mathrm{F}=\mathrm{B}+\mathrm{Dv}+\mathrm{Dl}$

$\mathrm{Qr}+\mathrm{F}^{*} \mathrm{Hf}=\mathrm{B}^{*} \mathrm{Hb}+\mathrm{Dv}^{*} \mathrm{Hdv}+\mathrm{Dl}^{*} \mathrm{Hdl}+\mathrm{Qc}$

$\mathrm{z} 1 * \mathrm{~F}=\mathrm{xb} 1 * \mathrm{~B}+\mathrm{ydl}{ }^{* \mathrm{D} v}+\mathrm{xd} 1 * \mathrm{Dl}$

Using these equations, the product recovery term can be expressed in terms of the constrained $(\mathrm{Y})$ and manipulated $(\mathrm{U})$ variables in order to calculate DPrDU. For example, the recovery for a bottoms product is:

$\operatorname{Pr}=B / F=(y d 1-x d 1) /(x b 1-y d 1) * D 1 / F+(z 1-y d 1) /(x b 1-y d 1)$

So if $U=y d 1$ then:

DPrDyd1 $=(z 1-x b 1) /(x b 1-y d 1) 2-D 1 / F 2 *(y d 1-x d 1) /(x b 1-y d 1) * D F / D y d 1+$

$$
1 / F^{*}(\mathrm{yd} 1-\mathrm{xd} 1) /(\mathrm{xb} 1-\mathrm{yd} 1) * \mathrm{DDl} / \mathrm{Dyd} 1+\mathrm{Dl} / \mathrm{F}^{*}(\mathrm{xb} 1-\mathrm{xd} 1) /(\mathrm{xb} 1-\mathrm{yd} 1) 2
$$

If $\mathrm{Dl}=0$ then this expression simplifies:

DPrDyd $1=(z 1-x b 1) /(x b 1-y d 1) 2$

To get the cost in the form above, first the reboiler heat Qr is expressed as a function of Qc:

$\mathrm{Qr}=\mathrm{B}^{*} \mathrm{Hb}+\mathrm{Dl}{ }^{*} \mathrm{Hdl}+\mathrm{Dv}^{*} \mathrm{Hdv}-\mathrm{F}^{*} \mathrm{Hf}+\mathrm{Qc}$

where the stream enthalpies are calculated assuming ideal mixing:

$\mathrm{Hf}=\mathrm{z}^{*} \mathrm{Hf} 1+\mathrm{z} 2 * \mathrm{Hf} 2$ 
$\mathrm{Hb}=\mathrm{xb} 1 * \mathrm{Hb} 1+\mathrm{xb} 2 * \mathrm{Hb} 2$

$\mathrm{Hdl}=\mathrm{xdl}{ }^{*} \mathrm{Hdl} 1+\mathrm{xd} 2 * \mathrm{Hdl} 2$

$\mathrm{Hdv}=\mathrm{ydl} * \mathrm{Hdv} 1+\mathrm{yd} 2 * \mathrm{Hdv} 2$

The energy equation is then rewritten using the product recovery definition. For example, if $\mathrm{Pr}=\mathrm{B} / \mathrm{F}$ and $\mathrm{Dv}=\mathrm{F}-\mathrm{B}-\mathrm{Dl}$ then:

$\mathrm{Qr}=\mathrm{F}^{*}[(\mathrm{Hb}-\mathrm{Hdv}) * \mathrm{Pr}+(\mathrm{Hdv}-\mathrm{Hf})]+\mathrm{Dl} \mathbf{l}^{*}(\mathrm{Hdl}-\mathrm{Hdv})+\mathrm{Qc}$

This $\mathrm{Qr}, \mathrm{Dv}=\mathrm{F}-\mathrm{B}-\mathrm{Dl}$, and $\mathrm{Pr}=\mathrm{B} / \mathrm{F}$ are substituted into the original cost function which when rearranged gives the form shown above.

\section{Run Options}

This menu choice SS Columns-Run runs the steady state distillation simulation with the train of columns in memory. The Low Level Controllers are part of the simulation and are always active unless the user resizes the index Yl vector to zero under Controllers - Indices. See Model Section for a discussion of the column model used in the simulation.

The program can run SMCO on a train of columns in two modes: independent and total. In the independent setting SMCO minimizes total costs using dynamic programming and the value of the interconnecting stream (Vi). In the independent mode, this intermediate value is calculated as the next column cost divided by the next column feed. For example, if the first column bottom stream feeds the second column, the value of the first column bottoms is the second column cost over the second column feed $(\mathrm{Vb}, 1=\mathrm{C} 2 / \mathrm{F} 2)$. The dynamic programming approach consists of optimizing the last column and then moving back up the train. When SMCO is in a total control mode, it assigns a value of zero to any intermediate streams and then minimizes the overall cost by considering the train as one unit.

To run Indep Mode choose SS Columns-Run-Indep Control or use the short-cut key Alt-1 for Indep Control. To run Total Mode choose SS Columns-Run-Total Control or use the short-cut key Alt-T for Total Control. In either case a dialog box will pop up asking the user to enter the number of time samples or steady state settling samples to run. During a run the current time sample is displayed in a box in the lower right corner right above the status line. If no such box is present then the simulation is not running. To abort or stop a run, just press any key on the keyboard.

If SMCO is running in the total control mode, it must construct vectors and matrices for the whole train. Since these train vectors and matrices are just 
combinations of the individual column vectors and matrices, they are constructed ONLY during the Total Control runs. This setup saves memory.

When a column is run in Independent Control with SMCO in manual, step testing can be performed. These step tests provide data to determine steady state gains (A) and partials needed in the control move calculation such as DYDF or DQcDU. When running in independent control with manual SMCO, the user will be prompted for changes in the column's manipulated variables (U). Note that only one column at a time in the train should be stepped, otherwise column interaction corrupts the data.

The Independent mode is preferable if some columns in the train have significantly different settling times. Using this mode allows SMCO on each column to update at the appropriate interval.

In the Total Control run mode, the SMCO Mode settings have no effect other than the predicted constrained variables (Ypred) of each column will be calculated only if its SMCO Mode is in auto.

The SMCO algorithm whether in Independent or Total Control can be summarized as follows:

$$
\Delta U=\left[A^{\mathrm{T}} \mathrm{W}^{\mathrm{T}} \mathrm{WA}(*)(\mathrm{I}+\Lambda)\right]^{-1}\left(\mathrm{~A}^{\mathrm{T}} \mathrm{W}^{\mathrm{T}} \mathrm{WA}-0.5 \mathrm{~W}_{\mathrm{c}} \mathrm{DCDU}\right)
$$

where:

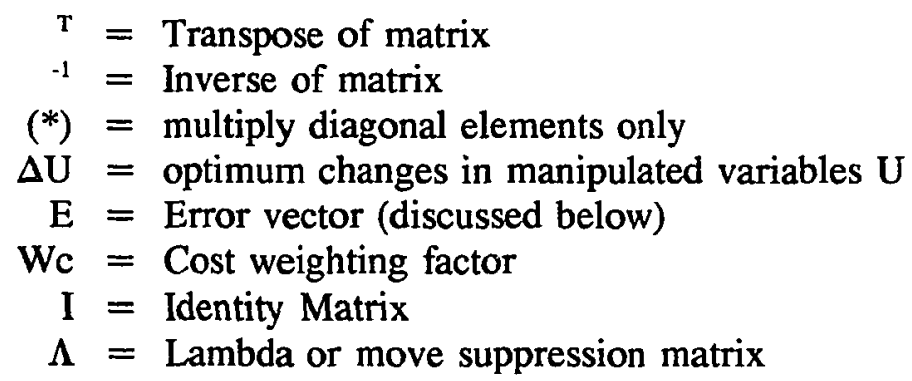

The SMCO calculation is normalized using the range of the constrained variables (Ymax-Ymin) and manipulated variables (Umax-Umin). The constraints on the process variables $(Y)$ can be viewed as soft constraints while those on the manipulated variables (U) can be thought of as hard constraints. The constraints on the manipulated variables (Umin, Umax) tell SMCO the maximum allowed range for the low-level controller set points. With a normalized function the $\Delta \mathrm{U}$ control moves are in fraction of range. 
The SMCO error is defined as the midpoint of the constrained range minus the current output $(E=Y$ mid - $Y)$. This definition represents two constraints with one term by symmetrically distributing the error over a valid range. This error is penalized by multiplying it by a function ( $f$ ) depending on how close the constrained variable gets to its limits. If the error penalty function is parabolic then $f=C * E^{2}$. The $\mathrm{C}$ constant is set so $\mathrm{f}$ is one at the minimum or maximum constraint $(\mathrm{C}=$ $\left.4 /(Y \max -Y \min )^{2}\right)$. For those values outside the constraint limits, $f$ is set to one. For a step penalty function, $f=0$ if within limits or $f=1$ if at or outside limits. The penalized errors are then normalized by dividing by the constraint range (YmaxY min).

\section{Misc Options}

One option under Misc is View file. This choice opens up a window and displays the first 120 lines of an ascii text file. On activating this option, a directory box appears where the user may either enter the name of the file to view or double click on a file in the Files box. When finished with a view file window, click the box in its upper left hand corner or press Alt-F3 to close the window.

The View Controls allows the user to view the various control vectors: $Y$ or the constrained variables, $\mathrm{U}$ or the manipulated variables, $\mathrm{Yl}$ or the Low Level Control variables, and the Low Level manipulated variables. NOTE that these vectors are built based on the information provided under Controllers-Indices. The View Controls is just for viewing. Altering any values under View Controls won't have a permanent effect.

\section{Graphs Options}

This menu item allows the user to work with Graphs and Outputs of an application.

First a graph needs to be created for a application. When creating a graph, first assign it a name if different than the default value. Next choose which of the seven possible plot variables to use to scale the left $Y 1$-axis and the right $\mathrm{Y} 2$-axis if the default value of 2 is unacceptable. Note that plot variable one should contain the independent variable or time. Next assign outputs to plot variables by highlighting the output then choosing the plot variable number 1-7, and pressing the Pick button. The current lo, hi, color, Yi-axis values will appear. Edit these values as desired. For example, the lo, hi fields can be used to scale the plot as desired. The Yi-axis field indicates which $\mathrm{Y}$-axis the variable is being plotted against (1 for left or Y1-axis and 2 for right or Y2-axis). Choose the desired color from black, blue, green, cyan, 
red, and magenta. The plot variables have the following symbols to help distinguish them when color is not available:

$\begin{array}{cl}\text { Plot Variable } & \text { Description } \\ 2 & \text { Plus sign (+) } \\ 3 & \text { Cross sign (x) } \\ 4 & \text { Y sign } \\ 5 & \text { Upside-down Y } \\ 6 & \text { Square Sign } \\ 7 & \text { Hour-Glass Sign }\end{array}$

Press OK when all values are as desired.

Edit Graph allows the user to modify the options discussed above once the graph has been created.

Write Data takes current Graph plot variable data and writes it in an ascii format to a file with the name of the graph with an extension of *.dat. This file can be imported into a spreadsheet or viewed for further analysis.

The delete option removes a graph that has been created.

The Plot option will display the current set of selected graphs on the screen. All output data up to the last value will be displayed. The screen may be printed using Print Screen provided the graphics command is executed from the dos prompt before running the program. To return to the desktop or clear the plot screen, press the SPACEBAR.

The Assign Display option allows the user to assign up to 4 plots from the application to be displayed when the program is run or when the plot option is chosen.

The Remove Display option removes a plot from the display set. The graph still exists but won't be displayed. This structure allows the user to choose up to 4 plots to be displayed either during a run or after a run. To permanently remove a graph, use the Delete option discussed above.

The Outputs option allow the user to edit and reset the outputs being used in the graphs. Each output has a path, name, units, and decimals. The decimals indicate the number of decimals used when displaying or storing the output. The reset option removes all past values of the outputs except for the most recent. NOTE that for EVERY run, new data is appended to the existing outputs. Without resetting, one may view several sets of run data for the same output. More information is given in the Outputs Section below. 


\section{Outputs}

This menu item allows the user to work with Outputs of the application.

Each application has a list of outputs used in plotting graphs and storing information. Each time a simulation is run, output data is stored on the disk in files with the startup output name plus some number extension like *.001. These files are temporary and created when the program first runs and deleted when it exits normally. If the program crashes, these output files will remain on disk and should be destroyed before re-running the program.

Choose the Edit option to modify the path, name, units, and decimals of each output.

The path is equivalent to a DOS path which tells the program where to store output information. At startup the root directory of the current disk is used. The same path is used for ALL OUTPUTS so changing the path of one output is like changing it for all of them. The output path can be changed BUT ALL the old outputs will be destroyed along with any data they contain and new outputs will be created in the new path. SO ONLY CHANGE THE OUTPUT PATH if don't need to look at old run data. For faster execution choose a path such as a ram drive or a hard disk. The output files are stored in the path with their name and a number extension like *.001, *.002, etc. Note that the output names should be limited to eight characters (DOS limitation).

The units field is simply to keep track of the current units of the given output for labelling purposes in the plotting of graphs.

The Decimals field is also used by the graph routine when labelling an axis or variable. The decimals tell the computer how many decimals to display for the given output.

Reset simply dumps all past output values from the output files. Each time the simulation is run, the outputs are updated by appending the new values to the existing files. This allows the user to compare data from different runs. By resetting the outputs of a column, previous run values will not be superimposed on the current ones.

\section{Help Option}

The last menu item provides Help windows on each of the menu bar choices. 


\section{Appendix C}

\section{PC Reactor/SMCO Simulation}

\section{Introduction}

The parallel reactor simulation used to generate results for Chapter 7 is written in Turbo Vision, an object-oriented language from Borland Pascal. Turbo vision provides the benefits of object programming in a window type environment for DOS. The program will run on any 386 or higher IBM compatible machine with $640 \mathrm{~K}$ and a CGA, EGA, or VGA compatible monitor. A mouse is not required but makes operating the program much easier. A disk with the raw code, executable, and sample data files is attached to the back cover. The reactor files on this disk are in a self-extracting executable file called Reactorl.exe. To extract the files, copy Reactorl.exe to another floppy or hard disk with $485 \mathrm{~K}$ free space and simply run the program which will generate the following files:

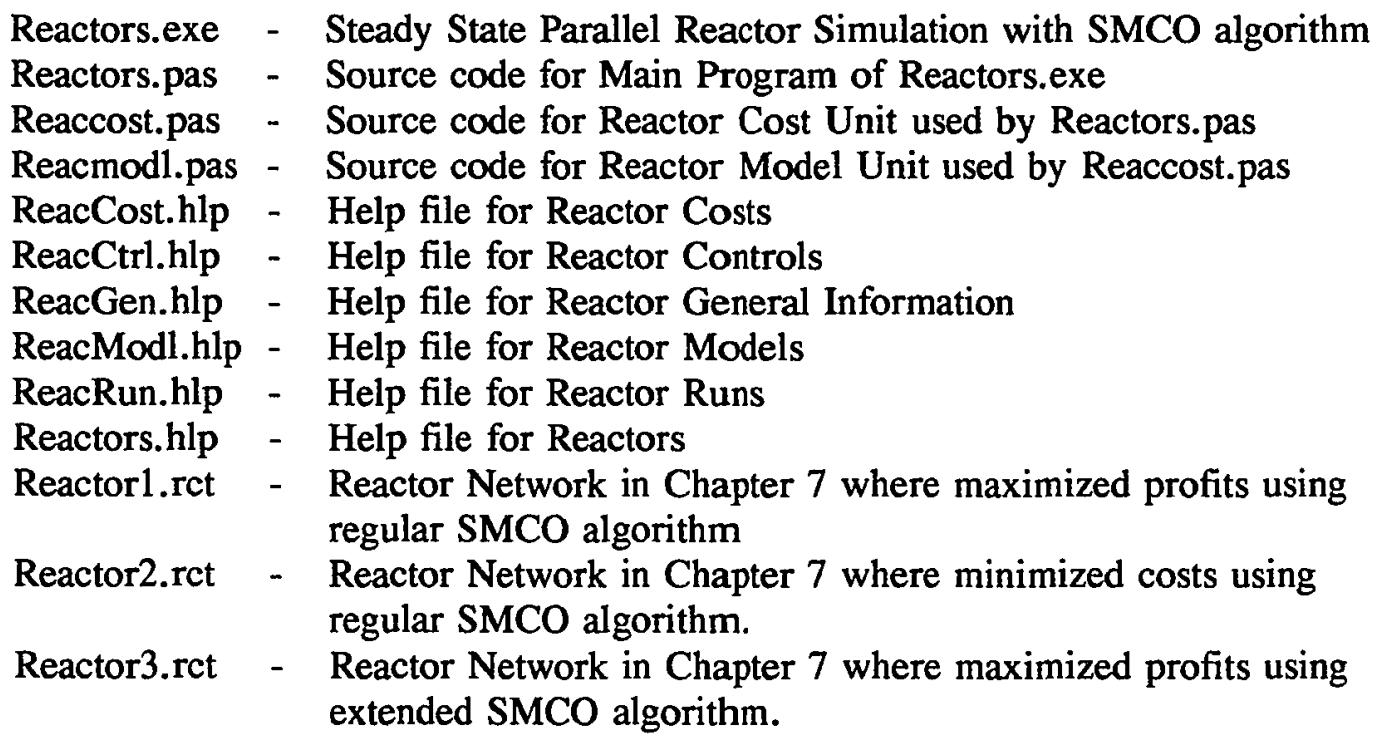

The self-extracting file can be deleted once the other files have been extracted.

Also contained on the disk is a self-extracting executable called Common.exe which contains common files used by the distillation and reactor simulations Extract these files to the same location as the reactor files noting that about $310 \mathrm{~K}$ of free space will be needed initially to hold the self-extracting executable and the extracted files. Again the self-extracting executable can be deleted once the files have been extracted. The files in Common.exe are: 
SSModel.pas - Source code for Steady State Model Unit

Output.pas - Source code for Output Unit used by SSModel.pas

Invert.pas - Source code for Invert Unit used by SSModel.pas

Vesa16.bgi - Graphics driver for Vesa16 screens

PC3270.bgi - Graphics driver for PC3270 screens

IBM8514.bgi - Graphics driver for IBM8514 screens

Herc.bgi - Graphics driver for Hercules screens

EGAVGA.bgi - Graphics driver for EGA or VGA screens

CGA.bgi - Graphics driver for CGA screens

ATT.bgi - Graphics driver for ATT screens

Litt.chr - Little font used by Graphic routines

Outputs.hlp - Help file for Outputs

Graphs.hlp - Help file for Graphs

Once the reactor and common files have been extracted to the same location, the Reactor/SMCO simulation can be run by entering REACTORS at the DOS prompt. The only files absolutely necessary to run the reactor program are reactors.exe, litt.chr, and the *.bgi files. The *.hlp files are only used if one tries to access Help on-line.

The following sections describe how the program is set up and what various options are available to the user. Note that some variable names are written to match those shown in the program. For example, $\mathrm{Vr}$ really represents $\mathrm{V}_{\mathrm{r}}$ or Fmin really represents $\mathrm{F}^{\mathrm{min}}$. The same information presented below is available on-line via the Help option on the Menu Bar.

\section{General Information}

Reactors.exe is a testing tool for the Supervisory Multivariable Constrained Optimization algorithm or SMCO for short. The goal of SMCO is to minimize costs while meeting design constraints for multivariable systems that come to steady state between samples. SMCO acts in a supervisory capacity directing lower level controllers (LLC).

The program has a Steady State Parallel Reactor simulation built in to test the performance of SMCO. See Model Section for more details.

When the program is started, a default reactor network exists. You may load another reactor set from a disk by choosing the Load option under SS Reactors. The program searches the current directory for files ending with $*$.rct.

The Misc Menu option contains the view file option which allows the user to view the first 120 lines of any ASCII or text file. 
See the other sections for more detailed information on each Menu item. Note that the Graph and Output Options are identical to those explained in Appendix B. The Help Option just brings up help windows on each menu selection or selected topic.

Any Menu option can be activated by pressing and holding the Alt key while pressing the highlighted letter of the desired menu option. The mouse may also be used to choose a menu item.

To clear a window just click the square in the upper left corner. If a mouse is unavailable, use the Windows menu option close (Alt-F3). Windows can be resized by dragging the lower right corner with the mouse or by choosing

Window-Size/Move (Ctrl-F5) and pressing the shift and arrow keys at the same time. To return back to the desktop press the ENTER key. To move a window just drag its title bar or choose Windows-Size/Move (Ctrl-F5) and use the arrow keys. Again press ENTER after finished moving a window with the keyboard. To enlarge a window to full screen click the arrow in the upper right corner or choose Window-Zoom (F5). To shrink the window back to its original size just click the arrow again or choose Window-Zoom (F5) again. The Window menu offers other options like close-all, cascade, and tile. The Next (F6) and Previous (Shift-F6) Window options jump from one window to the next.

For dialog boxes (windows with buttons and input boxes), press OK to accept the current data displayed. The dialog boxes will not go away until you either press OK or Cancel. Pressing the Escape key is like pressing the Cancel button. The default button is normally $O K$ and if you press the return key, it is like pressing the OK button with the mouse. You may use the TAB key to jump from one input box to another. Note you can shift-TAB to move backwards in the input blocks. You may also tab from one button to another.

\section{$\underline{\text { SS Reactor Options }}$}

This menu item allows you to work with Steady State Reactors in the network. Under Settings, the Unit Name can be changed. Note that this name may be up to 256 characters long but ONLY the first 8 characters are used as the file name for storage. The Output File specifies where output data is directed for each run. The data is stored as ASCII or text in a comma separated variable format. The file has an extension *.csv and can be read into almost any spreadsheet. The SMCO Mode indicates the status of the SMCO controller. See Run Section for more information. Use the Extended option to activate the extended controller during a run. The Error Penalty option indicates the function used to penalize constraint errors. 
The Output item under SS Reactors will bring up a window which displays current Reactor Output. The windows may be used to monitor the reactors' output during a run. Note, the values displayed in the Output Window are the same ones written to the Output File mentioned above.

The Run option runs the simulation. More information about running the program is under the Run Section.

The Load/Save option loads/saves the reactor network. When the reactor network is saved, all of its parts including graphs and outputs are also saved. An extension of *.rct is used for a reactor network file.

The Exit option quits the program.

\section{Controllers Options}

This menu item gives you access to the reactors' control variables which are grouped as:

(1) Indices

(2) Constraints

(3) Weights

(4) Matrices

(5) Values

(6) Cost Data

Indices consist of:

Uon $=$ integer vector for active (1) or inactive (2) manipulated variables

Constraints consist of:

$$
\begin{aligned}
\text { Rmin } & =\text { Minimum Raw Feed Constraints for SMCO } \\
\text { Rmax } & =\text { Maximum Raw Feed Constraints for SMCO } \\
\text { Fmin } & =\text { Minimum Reactor Feed Limit } \\
\text { Fmax } & =\text { Maximum Reactor Feed Limit } \\
\text { Pmin } & =\text { Minimum Product Rate Constraints for SMCO } \\
\text { Pmax } & =\text { Maximum Product Rate Constraints for SMCO }
\end{aligned}
$$

Note that $\mathrm{R}$ and $\mathrm{P}$ are the constrained variables and $\mathrm{F}$ are the manipulated variables.

Weights consist of:

$$
\mathrm{W}=\text { SMCO Weights for Constrained } \mathrm{Y} \text { variables }
$$

Lambda = SMCO Lambda or move suppression parameters 
Matrices consist of:

$\mathrm{B}=$ Breakdown matrix of reactor feeds

$\mathrm{Q}=$ Product quotas or yields

$\mathrm{A}=$ SMCO Steady State Gains

Values consist of:

$\mathrm{R}=$ Raw Feed Rates in $\mathrm{Klb} / \mathrm{hr}$

$\mathrm{F}=$ Reactor Feed Rates in Klb/hr

$\mathrm{P}=$ Product Flow Rates in $\mathrm{Klb} / \mathrm{hr}$

See Model Section for more information on reactor model.

Cost Data consist of:

DCDU = Derivatives of Cost Fnc. wrt manipulated variables U

$\mathrm{Vr}=$ Value of Raw Feeds in $\$ / \mathrm{Klb}$

$\mathrm{Vp}=$ Value of Product Streams in $\$ / \mathrm{Klb}$

For more information on the reactor cost, see Cost Section below.

\section{Cost Definition}

The cost function for the reactor simulation is written as a sum of the raw feeds and product streams weighted by their economic values:

$$
\text { Cost }=\sum_{k} V_{r k} R_{k}+\sum_{i} V_{p i} P_{i}
$$

where:

$$
\begin{aligned}
V_{r k} & =\text { value of raw feed } \mathrm{k} \text { in } \$ / \mathrm{klb} \\
\mathrm{R}_{\mathrm{k}} & =\text { raw feed } \mathrm{k} \text { in } \mathrm{klb} / \mathrm{hr} \\
\mathrm{V}_{\mathrm{pi}} & =\text { value of product } \mathrm{i} \text { in } \$ / \mathrm{klb} \\
\mathrm{P}_{\mathrm{i}} & =\text { product } \mathrm{i} \text { rate in } \mathrm{klb} / \mathrm{hr}
\end{aligned}
$$

Fuel costs are assumed negligible and not included in the cost formulation. All flow streams carry a positive sign while their values $(\mathrm{Vr}, \mathrm{Vp})$ reflect either a profit, if negative, or a debit, if positive.

The Product Rates P[i] can be written as:

$$
P_{i}=\sum_{k} Q_{i, k} R_{k}
$$


where:

$$
\mathrm{Q}_{\mathrm{i}, \mathrm{k}}=\text { quotas or yields in } \mathrm{lb} \text { prod } \mathrm{i} / \mathrm{lb} \text { raw feed } \mathrm{k}
$$

Substituting this relation and rearranging gives total cost of:

$$
\text { Cost }=\sum_{k}\left(V_{r k}+\sum_{i} Q_{i, k} V_{p i}\right) R_{k}
$$

Which can then be written as:

$$
\text { Cost }=\sum_{k} C_{k} R_{k}
$$

where:

$$
C_{k}=V_{r k}+\sum_{i} Q_{i, k} V_{p i}
$$

$\mathrm{C}_{\mathrm{k}}$ can be viewed as the operating cost per klb raw feed $\mathrm{k}$.

For cost derivatives need Cost in terms of manipulated variables or Reactor feeds F[j]. To accomplish this task use raw feed model:

$$
R_{k}=\sum_{j} B_{k j} F_{j}
$$

where:

$\mathrm{B}_{\mathrm{k}, \mathrm{j}}=$ breakdown of feeds in $\mathrm{lb}$ raw feed $\mathrm{k} / \mathrm{lb}$ reactor feed $\mathrm{j}$

So the total cost can be written as:

$$
\text { Cost }=\sum_{k}\left(\sum_{j} B_{k j} F_{j}\right) C_{k}
$$

So the cost partial becomes:

$$
\frac{\partial \operatorname{Cos} t}{\partial F_{j}}=\sum_{k} B_{k j} C_{k}
$$

The program will evaluate all these terms based on the matrices $B$ and $Q$ that the user provides. See Model Section for more information. 


\section{Run Options}

This menu choice SS Reactors-Run runs the steady state parallel reactor simulation. See Help-Model for a discussion of the reactor model used in the simulation.

To run the Reactor Simulation choose SS Columns-Run or use the short-cut key Alt-R for Run. A dialog box will pop up asking the user to enter the number of time samples or steady state settling samples to run. During a run the current time sample is displayed in a box in the lower right corner right above the status line. If no such box is present then the simulation is not running. To abort or stop a run, just press any key on the keyboard.

The SMCO algorithm whether in Independent or Total Control can be summarized as follows:

$$
\Delta \mathrm{U}=\left[\mathrm{A}^{\mathrm{T}} \mathrm{W}^{\mathrm{T}} \mathrm{WA}\left({ }^{*}\right)(\mathrm{I}+\Lambda)\right]^{-1}\left(\mathrm{~A}^{\mathrm{T}} \mathrm{W}^{\mathrm{T}} \mathrm{WA}-0.5 \mathrm{~W}_{\mathrm{c}} \mathrm{DCDU}\right)
$$

where:

$$
\begin{aligned}
\mathrm{T} & =\text { Transpose of matrix } \\
-1 & =\text { Inverse of matrix } \\
\left({ }^{*}\right) & =\text { multiply diagonal elements only } \\
\Delta \mathrm{U} & =\text { optimum changes in manipulated variables } \mathrm{U} \\
\left(^{*}\right) & =\text { multiply diagonal elements only } \\
\mathrm{E} & =\text { Error vector (discussed below) } \\
\mathrm{Wc} & =\text { Cost weighting factor } \\
\mathrm{I} & =\text { Identity Matrix } \\
\Lambda & =\text { Lambda or move suppression matrix }
\end{aligned}
$$

The SMCO calculation is normalized using the range of the constrained variables (Ymax-Ymin) and manipulated variables (Umax-Umin). For the reactor network, the raw feed flows and product rates are the $Y$ constrained variables and the reactor feeds are the $U$ manipulated variables. The constraints on the process variables $(Y)$ can be viewed as soft constraints while those on the manipulated variables (U) can be thought of as hard constraints. The constraints on the manipulated variables (Umin, Umax) tell SMCO the maximum allowed range for the low-level controller set points. With a normalized function the $\Delta U$ control moves are in fraction of range.

The SMCO error is defined as the midpoint of the constrained range minus the current output ( $E=$ Ymid - $Y$ ). This error is penalized by multiplying it by a function ( $f$ ) depending on how close the constrained variable gets to its limits. If the error penalty function is parabolic then $f=C^{*} E^{2}$. The $C$ constant is set so $f$ is one at the minimum or maximum constraint $\left(C=4 /(Y \max -Y \min )^{2}\right)$. For those values 
outside the constraint limits, $f$ is set to one. For a step penalty function, $f=0$ if within limits or $f=1$ if at or outside limits. The penalized errors are then normalized by dividing by the constraint range (Ymax- Ymin). 


\section{Vita}

The author was born in Augusta, Georgia on May 11, 1964 to Mr. and Mrs. William E. Daniel. He was raised in North Augusta, South Carolina, where he received his elementary and high school education. While at North Augusta Senior High, the author became interested in chemistry, physics, and mathematics, and after graduating in June 1982, he enrolled at Clemson University in South Carolina. At Clemson he majored in chemical engineering and took some courses that peaked his interest in process control. After graduating from Clemson with a Bachelor of Science in Chemical Engineering in May 1987, the author enrolled at the University of Maryland to further his knowledge and understanding of process control. At Maryland, he completed a Master of Science under the direction of Dr. O. A. Asbjornsen in June 1990. The title of his thesis was "Constrained Predictive Control in View of Natural Process Disturbances." His interests in control then brought him to Louisiana State University where he completed a Doctor of Philosophy under the guidance of Dr. A. B. Corripio in December 1993. The dissertation was entitled "An Algorithm for Supervisory Multivariable Constrained Optimization of Industrial Processes." The author plans to get a job in industry in an area concerning process control, such as design and development. 


\title{
DOCTORAL EXAMINATION AND DISSERTATION REPORT
}

\author{
Candidate: William E. Daniel, Jr.
}

Major Field: Chemical Engineering

Title of Dissertation: An Algorithm for Supervisory Multivariable Constrained Optimization of Industrial Processes

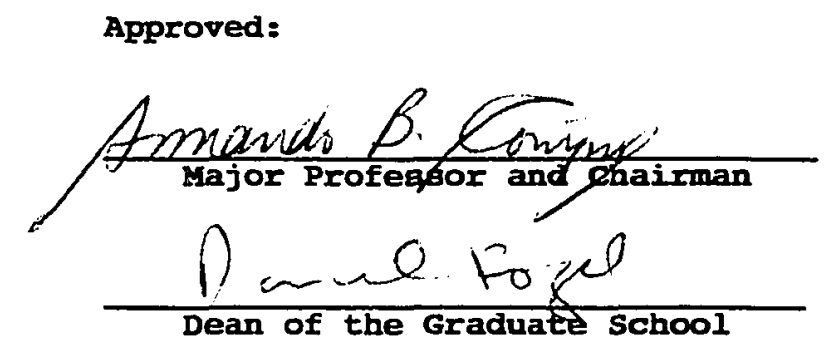

EXAMINING COMMITTEE:

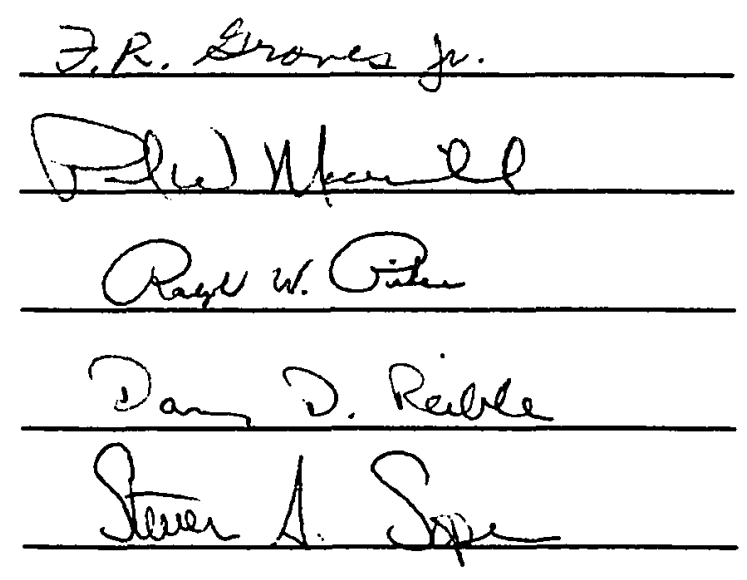

Date of Examination:

November 2, 1993

Reproduced with permission of the copyright owner. Further reproduction prohibited without permission. 